\title{
Fast wavelet collocation methods for second kind integral equations on polygons
}

Yi Wang

West Virginia University

Follow this and additional works at: https://researchrepository.wvu.edu/etd

\section{Recommended Citation}

Wang, Yi, "Fast wavelet collocation methods for second kind integral equations on polygons" (2003). Graduate Theses, Dissertations, and Problem Reports. 1904.

https://researchrepository.wvu.edu/etd/1904

This Dissertation is protected by copyright and/or related rights. It has been brought to you by the The Research Repository @ WVU with permission from the rights-holder(s). You are free to use this Dissertation in any way that is permitted by the copyright and related rights legislation that applies to your use. For other uses you must obtain permission from the rights-holder(s) directly, unless additional rights are indicated by a Creative Commons license in the record and/ or on the work itself. This Dissertation has been accepted for inclusion in WVU Graduate Theses, Dissertations, and Problem Reports collection by an authorized administrator of The Research Repository @ WVU.

For more information, please contact researchrepository@mail.wvu.edu. 


\title{
Fast Wavelet Collocation Methods for Second Kind Integral Equations on Polygons
}

\author{
Yi Wang \\ Dissertation submitted to the \\ College of Arts and Sciences \\ at West Virginia University
}

in partial fulfillment of the requirements for the degree of

Doctor of Philosophy

in

Mathematics

Yuesheng Xu, Ph.D., Chair

Martina E. Bachlechner, Ph.D.

Harvey Diamond, Ph.D.

Weifu Fang, Ph.D.

Sherman D. Riemenschneider, Ph.D.

2003

Morgantown, West Virginia

Keywords: fast collocation methods, Fredholm integral equations of the second kind, refinable sets, multiscale interpolation, wavelets

AMS subject classifications: 65B05, 45L10

Copyright 2003, Yi Wang 


\section{Fast Wavelet Collocation Methods for Second Kind Integral Equations on Polygons}

Yi Wang

$(\mathrm{ABSTRACT})$

In this thesis we develop fast wavelet collocation methods for integral equations of the second kind with weakly singular kernels over polygons. For this purpose, we construct multiscale wavelet functions and collocation functionals having vanishing moments. Moreover, we propose several truncation strategies, which lead to fast algorithms, for the coefficient matrix of the corresponding discrete system. Critical issues for numerical implementation of such methods are considered, such as choices of practical truncation strategies, numerical integration of weakly singular integrals, error controls of numerical quadrature and numerical solutions of resulting compressed linear systems. Numerical experiments are given to demonstrate proposed ideas and methods. Finally, parallel computing using developed methods is investigated.

That this work received partial support from the US NSF grant EPSCoR-0132740. 


\section{Acknowledgments}

I would like to thank my advisor, Dr. Yuesheng $\mathrm{Xu}$, for his excellent guidance, advice and continual encouragement. It is my great pleasure to work under his supervision. Without him, this dissertation could not have come out.

I would also like to thank Dr. Sherm Riemenschneider for his many helps and supports for my research, especially in parallel computing. I also would like to acknowledge my indebtedness to Dr. Weifu Fang for his support of my first year at West Virginia University and many valuable discussions, to my other committee members: Dr. Harvey Diamond and Dr. Martina E. Bachlechner for their help during my studies.

My thanks also go to all professors of West Virginia University and North Dakota State University and all of my friends who have given me help either in my studying, or in my ordinary life.

Finally, I would like to thank my wife, Qun, my daughter Meili, my Mom, Daddy and my whole family for their loves and their supports. 


\section{Contents}

1 Introduction $\quad 1$

2 Fast Wavelet Collocation Methods $\quad 4$

2.1 Multiscale Collocation Schemes . . . . . . . . . . . . . . . . 4

2.2 General Construction of Multiscale Functions and Functionals . . . . . . . . 7

3 Wavelets and Collocation Functionals on the Unit Triangle 12

3.1 The Approximation Spaces . . . . . . . . . . . . . . . . . . . . 14

3.2 Initial Wavelets in $\mathbb{W}_{1} \ldots \ldots \ldots \ldots \ldots \ldots$

3.3 Initial Collocation Functionals in $L_{1} \ldots \ldots \ldots \ldots \ldots \ldots$

3.4 Properties of the Wavelet Basis Functions and the Collocation Functionals . 26

3.5 Reconstruction of Functions . . . . . . . . . . . . . . . . . 30

4 Computation of Discrete Systems and Their Solutions 32

4.1 The Matrix $\mathbf{E}_{n} \ldots \ldots \ldots \ldots \ldots$

4.2 The Matrix $\mathbf{K}_{n} \ldots \ldots \ldots \ldots \ldots \ldots \ldots$

4.3 An Adaptive Quadrature Rule . . . . . . . . . . . . . . . . . . . . . . . . 42

4.4 Error Analysis of the Adaptive Quadrature Rule . . . . . . . . . . . . . . 47 
4.5 Compression Schemes-Distance Computing . . . . . . . . . . . . . . 55

4.6 Block Truncation Schemes . . . . . . . . . . . . . . . . . 66

4.7 Quadrature Error Control ....................... 73

4.8 Iteration Methods . . . . . . . . . . . . . . . . . . . . . 79

5 Fast Wavelet Collocation Methods on Polygonal Domains $\quad 88$

5.1 On General Triangles . . . . . . . . . . . . . . . . 88

5.2 On Polygons . . . . . . . . . . . . . . . . . . . . . . . . . . 90

5.3 Computation of Matrix Elements . . . . . . . . . . . . . . . . . . 94

5.4 Properties of the Wavelet Basis functions and the Collocation Functionals Over Polygons . . . . . . . . . . . . . . . . . . . . . . 95

$6 \quad$ Numerical Experiments for Solving Integral Equations 102

6.1 On the Unit Triangle . . . . . . . . . . . . . . . . . 102

6.2 On General Triangles . . . . . . . . . . . . . . . . . . . . . . . . . . 104

6.3 On Polygons . . . . . . . . . . . . . . . . . . 106

6.4 Parallel Computing . . . . . . . . . . . . . . . . . . 108 


\section{List of Figures}

2.1 Multiscale partitions of the unit triangle $\Omega \ldots \ldots \ldots$

3.1 Original domain-unit triangle $\Omega \ldots \ldots \ldots \ldots \ldots$

3.2 Scaling function $w_{00} \ldots \ldots \ldots \ldots \ldots \ldots \ldots$

3.3 Scaling function $w_{01} \ldots \ldots \ldots \ldots \ldots \ldots \ldots$

3.4 Scaling function $w_{02} \ldots \ldots \ldots \ldots \ldots \ldots \ldots$

3.5 Wavelet function $w_{10} \ldots \ldots \ldots \ldots \ldots \ldots$

3.6 Wavelet function $w_{11} \ldots \ldots \ldots \ldots \ldots \ldots \ldots$

3.7 Wavelet function $w_{12} \ldots \ldots \ldots \ldots \ldots \ldots \ldots \ldots \ldots$

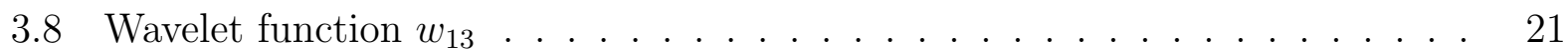

3.9 Wavelet function $w_{14} \ldots \ldots \ldots \ldots \ldots \ldots \ldots \ldots$

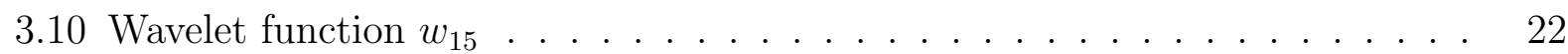

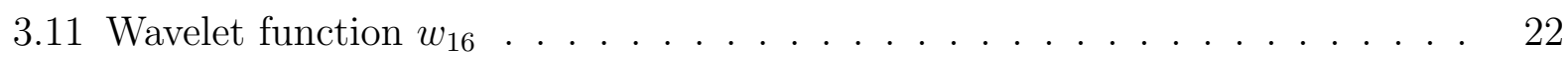

3.12 Wavelet function $w_{17} \ldots \ldots \ldots \ldots \ldots \ldots \ldots$

3.13 Wavelet function $w_{18} \ldots \ldots \ldots \ldots \ldots \ldots \ldots \ldots$

3.14 Collocation points of $L_{1} \ldots \ldots \ldots \ldots \ldots \ldots \ldots \ldots$

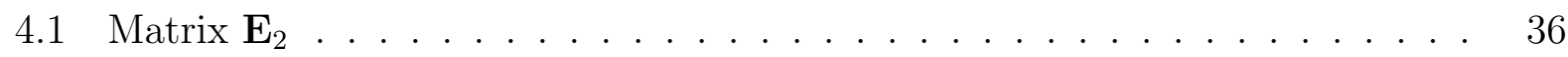




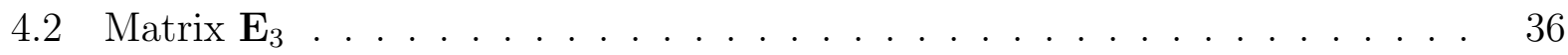

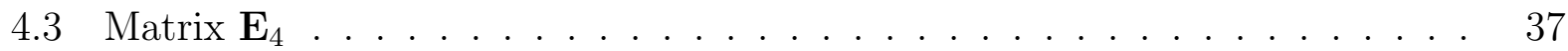

4.4 Domains of integration . . . . . . . . . . . . . . . . . . . 41

4.5 Full Matrix $\mathbf{K}_{3} \ldots \ldots \ldots \ldots$

4.6 Domain of integration for Example $4.9 \ldots \ldots \ldots$. . . . . . . . . . . 51

4.7 Domain of integration for Example $4.10 \ldots \ldots \ldots$

4.8 Compression rates of different strategies . . . . . . . . . . . . . . . . . . 57

4.9 Convergent rates of different strategies . . . . . . . . . . . . 58

4.10 Convergent rates of strategy 3 with different $\nu \ldots \ldots$. . . . . . . . 61

4.11 Convergent rates of strategy 2 with different $\nu \ldots \ldots \ldots$. . . . . . 62

4.12 The sparse matrix $\mathbf{K}_{3}$ after compression $\ldots \ldots \ldots$. . . . . . . . 63

4.13 The sparse matrix $\mathbf{K}_{4}$ after compression . . . . . . . . . . . . . 63

4.14 The sparse matrix $\mathbf{K}_{5}$ after compression . . . . . . . . . . . . . 64

4.15 The Full matrix $\mathbf{K}_{3}$ and the compressed matrix $\tilde{\mathbf{K}}_{3} \ldots \ldots \ldots$

4.16 Distance of two triangles $S_{i^{\prime} j^{\prime}}$ and $S \ldots \ldots \ldots \ldots$

4.17 Block truncation scheme . . . . . . . . . . . . . . . . . . . . . . 70

5.1 The affine mapping $T_{k} \ldots \ldots \ldots \ldots \ldots$

6.1 Computational domains of Example 6.5 and Example $6.7 \ldots \ldots$. . . . . 107

6.2 Time needed vs. number of processes: level $n=5 \ldots \ldots$. . . . . . . . 114

6.3 Time needed vs. number of processes: level $n=7 \ldots \ldots$. . . . . . . 114 


\section{List of Tables}

4.1 Quadrature computing results for Example $4.9 \ldots \ldots$. . . . . . . . . 52

4.2 Quadrature computing results for example $4.10 \ldots \ldots$. . . . . . . . . . 53

4.3 Comparison of uncompressed solutions by the wavelet collocation method, with and without numerical quadratures for matrix entries. . . . . . . . . 54

4.4 Comparison of compression rates of different strategies . . . . . . . . . 56

4.5 Comparison of solutions obtained by the full collocation method and by our compressed collocation method (strategy 1 with $\nu=1$ ) . . . . . . . . 59

4.6 Comparison of solutions obtained by the full collocation method and by our compressed collocation method (strategy 2 with $\nu=1$ ). . . . . . . . . 59

4.7 Comparison of solutions obtained by the full collocation method and by our compressed collocation method (strategy 3 with $\nu=1$ ) . . . . . . . . . 60

4.8 Comparison of solutions obtained from strategy 3 with different $\nu \ldots . . .60$

4.9 Comparison of solutions obtained from strategy 2 with different $\nu \ldots$. . . 60

4.10 Comparison of solutions obtained from strategy 2 with different $\nu \ldots . \ldots 1$

4.11 Comparison of solutions obtained by the full collocation method and by the compressed method (with block truncation scheme) . . . . . . . . . . . 74

4.12 Comparison of uncompressed solutions, with and without numerical quadratures, using different $m_{n}$ for different $n \ldots \ldots \ldots \ldots$ 
4.13 Comparison of uncompressed solutions, with and without numerical quadratures, using different numbers of subdivisions for different levels. . . . . . . . 80

4.14 Comparison of uncompressed solutions using Gaussian elimination and multilevel iteration methods. . . . . . . . . . . . . . . . . . . . .

6.1 Comparison of compressed solutions using multilevel iteration method, with and without quadrature rule, number of iteration $=15 \ldots \ldots \ldots$

6.2 Comparison of compressed solutions using multilevel iteration method, with and without quadrature rule, number of iteration=15 . . . . . . . . . 104

6.3 Comparison of solutions obtained by the full collocation method and by our compressed collocation method (strategy 2, on a general triangle). . . . . . . 105

6.4 Comparison of solutions obtained by the full collocation method and by our compressed collocation method (block truncation, on a general triangle). . . 106

6.5 Comparison of solutions obtained by the full collocation method and by our compressed collocation method (strategy 2, on a quadrangle). . . . . . . . 107

6.6 Comparison of solutions obtained by the full collocation method and by our compressed collocation method (block truncation, on a quadrangle). . . . . . 108

6.7 Comparison of solutions obtained by the full collocation method and by our compressed collocation method (strategy 2, on a pentagon). . . . . . . . . 109

6.8 Comparison of solutions obtained by the full collocation method and by our compressed collocation method (block truncation, on a pentagon). . . . . . . 109

6.9 Comparison of times consumed when number of processes varies. . . . . . . . 113 


\section{Chapter 1}

\section{Introduction}

In this thesis we develop fast wavelet collocation methods for solving Fredholm integral equations of the second kind on polygons. We consider integral equations of the form

$$
u(t)-\int_{E} K(s, t) u(s) d s=f(t), \quad t \in E,
$$

where $E$ is a polygon in $\mathbb{R}^{2}$, and the kernel $K(s, t)$ is a weakly singular function on $E \times E$. Introducing an operator

$$
(\mathcal{K} u)(t)=\int_{E} K(s, t) u(s) d s, \quad t \in E,
$$

(1.1) can be written into

$$
(\mathcal{I}-\mathcal{K}) u=f .
$$

This type of equations has many important applications including boundary integral equations [At1, At2] and radiosity equations [ACr, AC2]. There have been many theoretical studies as well as numerical methods on such Fredholm integral equations of the second kind (cf. $[\mathrm{At} 2, \mathrm{~K}]$ ).

Using a conventional method such as Galerkin method or collocation method to discretize an integral equation will lead to a linear system with a full coefficient matrix. It's computationally costly to generate the full coefficient matrix. Especially, when higher dimensional integral equations are considered, the computational cost to generate the full coefficient matrix is formidable. Therefore fast algorithms are extremely crucial for numerical solutions of 
integral equations. Recently, wavelet methods have been playing important roles in seeking fast algorithms for numerical solutions of integral equations.

Wavelet Galerkin methods and Petrov-Galerkin methods were investigated recently by $[\mathrm{Al}$, BCR, CMX1, CMX2, DPS1, DPS2, MXZ, PS, PSS, R] as well as references cited therein. A fast multilevel method was developed in [CMX4] using a multilevel decomposition of the approximation space. [DPS2, MXZ] studied compression strategies with slightly different focuses. [DPS2] studied wavelet Galerkin methods using periodic wavelets based on refinement equations for periodic problems, while [MXZ] developed wavelet Galerkin methods using piecewise polynomial wavelets. One advantage of using piecewise polynomial wavelets is that the wavelet functions have close forms which provide convenience for computation. The implementation of wavelet Galerkin methods based on refinement equations was done in [DKPS]. The implementation of wavelet Galerkin methods based on piecewise polynomial wavelets was done in $[\mathrm{FWX}]$. These methods use $L_{2}$ analysis and therefore the vanishing moments of the multiscale basis functions naturally lead to matrix truncation schemes.

Collocation methods due to lower computational cost for evaluations of integrals (for example , see $[\mathrm{At1}, \mathrm{AC} 1, \mathrm{CX}])$, receive more favorable attention from engineering fields. Yet less attention has been paid to them. For collocation methods, the appropriate space to work in is $L_{\infty}$ and, this causes challenging technical obstacles for identifying good matrix truncation strategies. The recent major work in this area is done in [CMX3]. [CMX3] provides a theoretical framework of fast wavelet collocation methods for solving integral equations on invariant domains associated with families of contractive mappings. [CWX2] studies the actual computation of 1-D integral equations using methods proposed in [CMX3].

Based on the framework discussion in [CMX3], it is the purpose of this thesis to investigate actual computing methods for 2-D wavelet collocation methods. By constructing semi-biorthogonal wavelets and collocation functionals for 2-D collocation scheme, practical matrix truncation strategies are studied. Theses practical strategies lead to fast algorithms for solving integral equations. The $2-D$ wavelets are constructed on invariant domains and have simple expressions. Both wavelet basis functions and associated collocation functionals have vanishing moments of certain degrees and multilevel structures. The collocation func- 
tionals are constructed through set wavelets. Results from the truncated system have the same accuracy and convergence order as those from uncompressed ones, yet the computing cost is dramatically reduced from $O\left(N^{2}\right)$ to $O(N \log N)$, where $N$ is the dimension of the approximation space. The approximation solution has a convergence order of $O\left(N^{k / 2} \log N\right)$, where $k$ is the total degree of piecewise polynomials in the approximation space. The condition number of the compressed coefficient matrix is of order $O\left(\log ^{2} N\right)$. The methods developed in this thesis have application not only to the integral equations, but also can be used to solve certain 3-D boundary value problems.

More specifically, In chapter 2 we briefly review the methods proposed in [CMX3]. In Chapter 3, we develop our wavelet collocation methods on the unit triangle. The multilevel wavelet basis and collocation functionals on the unit triangle are constructed. Properties of the constructed wavelets and collocation functionals are also investigated. A reconstruction algorithm of the sought function is also discussed in this chapter. In Chapter 3, we discuss how to compute the coefficient matrix in detail. Other critical issues are considered too, such as, compressing schemes, computation of integrals with singular kernels, and solutions of the resulting linear system. We provide two practical truncation strategies based on the theoretical truncation strategy proposed in [CMX3]. An efficient adaptive quadrature rule for computing singular double integrals is also proposed. The control of errors introduced from the numerical integration is analyzed. Moreover, we develop a multilevel iterative method solving resulting sparse linear systems by adapting ideas proposed in [FMX]. In Chapter 5, fast wavelet methods on polygons are developed based on methods on the unit triangle and triangulations of polygons. In Chapter 6, we combine all proposed methods together to solve a number of examples to demonstrate accuracies and efficiencies of proposed methods. Parallel computing algorithms and their implementation in a Beowulf cluster are also investigated and demonstrated. 


\section{Chapter 2}

\section{Fast Wavelet Collocation Methods}

\subsection{Multiscale Collocation Schemes}

In this chapter we briefly review the methods developed in [CMX3] for solving Fredholm integral equations of the second kind. Let $E$ be a compact set with star shape in the $d$ dimensional Euclidean space $\mathbb{R}^{d}$, and let $\mathbb{X}=L^{\infty}(E), \mathbb{V}=C(E)$. Suppose that $K$ is a weakly singular kernel, that is, for every $s \in E, K(s,.) \in L^{1}(E)$. Therefore, the operator $\mathcal{K}: \mathbb{X} \rightarrow \mathbb{V}$ defined by

$$
(\mathcal{K} u)(s):=\int_{E} K(s, t) u(t) d t, s \in E
$$

is compact in $\mathbb{X}$. We consider Fredholm integral equations of the second kind

$$
u-\mathcal{K} u=f,
$$

where $f \in \mathbb{X}$ is a given function and $u \in \mathbb{X}$ is the unknown to be determined. By Fredholm alternative theorem, when the corresponding homogeneous equation has only trivial solution, equation (2.2) has a unique solution in $\mathbb{X}$.

Let the index set $\mathbb{N}_{0}:=\{0,1, \ldots\}$. Assume that a sequence of approximation spaces $\mathbb{F}_{n}, n \in$ $\mathbb{N}_{0}$ of $\mathbb{X}$, satisfying

$$
\mathbb{F}_{n} \subseteq \mathbb{F}_{n+1}, \quad n \in \mathbb{N}_{0}
$$


and

$$
\mathbb{V} \subseteq \overline{\bigcup_{n \in \mathbb{N}_{0}} \mathbb{F}_{n}}
$$

By the nestedness $(2.3) \mathbb{F}_{n}$ can be further decomposed into the direct sum of a family of spaces

$$
\mathbb{F}_{n}=\mathbb{W}_{0} \oplus \mathbb{W}_{1} \oplus \ldots \oplus \mathbb{W}_{n}
$$

where spaces $\mathbb{W}_{n}$ have multiscale structures and will be constructed as piecewise polynomial functions on $E$. Note $\mathbb{F}_{0}=\mathbb{W}_{0}$. Let $w(n):=\operatorname{dim} \mathbb{W}_{n}$ and, we have that

$$
f(n):=\operatorname{dim} \mathbb{F}_{n}=\sum_{r \in \mathbb{Z}_{n+1}} w(r),
$$

where, $\mathbb{Z}_{m}:=\{0,1, \ldots, m-1\}$. A multiscale partition of the set $E$ is also needed for the wavelet collocation method. It consists of a family of partitions $\left\{E_{n}: n \in \mathbb{N}_{0}\right\}$ of $E$. For each $n \in \mathbb{N}_{0}$ the partition $E_{n}$ consists of a family of subsets $\left\{E_{n i}: i \in \mathbb{Z}_{e(n)}, e(n)=c w(n), n \geq 0\right\}$ of $E$ with the properties

$$
\operatorname{meas}\left(E_{n i} \cap E_{n i^{\prime}}\right)=0, \quad i, i^{\prime} \in \mathbb{Z}_{e(n)}, i \neq i^{\prime},
$$

and

$$
\bigcup_{i \in \mathbb{Z}_{e(n)}} E_{n i}=E
$$

Where $c$ is a generic constant in this thesis. (We sometimes also use $C$ for the generic constant). When $n=0$ the set $E$ is the only cell in the partition. See Fig. 2.1 for an example of partitions when $E$ is the unit triangle. Further, the partition $E_{n}$ has the following property. Namely, for some positive constants $c_{-}, c_{+}$and all $n \in \mathbb{N}_{0}$

$$
c_{-} \mu^{-n / d} \leq \max \left\{d\left(E_{n, i}\right): i \in \mathbb{Z}_{e(n)}\right\} \leq c_{+} \mu^{-n / d}
$$

where $d$ is the spatial dimension, and $\mu$ is a constant positive integer. For a set $A \subset \mathbb{R}^{d}, d(A)$ represents the diameter of $A$, i.e.,

$$
d(A):=\sup \{|x-y|: x, y \in A\}
$$

and |.| denotes the Euclidean norm on the space $\mathbb{R}^{d}$. The family of partitions $\left\{E_{n}: n \in \mathbb{N}_{0}\right\}$ is used to specify supports of both basis functions and linear functionals. Specifically, assume

$$
\mathbb{W}_{n}:=\operatorname{span} W_{n}, \quad n \in \mathbb{N}_{0}
$$




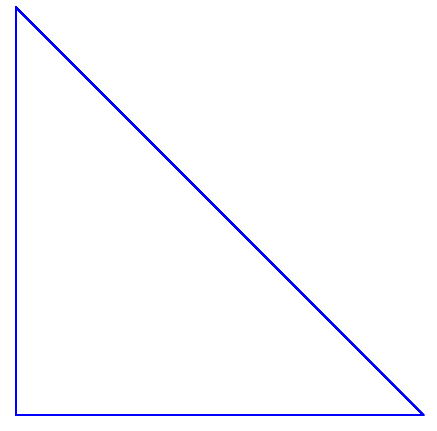

$\mathrm{n}=0$

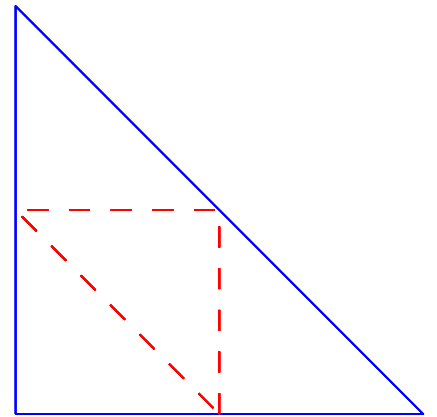

$n=1$

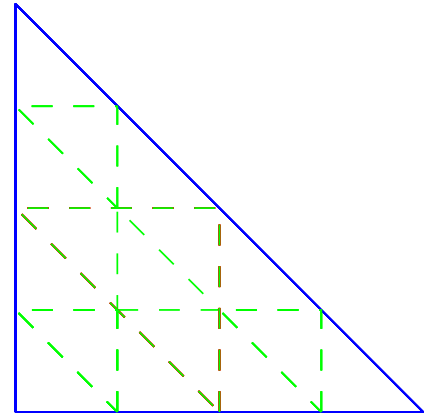

$n=2$

Figure 2.1: Multiscale partitions of the unit triangle $\Omega$

where, $W_{n}:=\left\{w_{n m}: m \in \mathbb{Z}_{w(n)}\right\}$. The basis function $w_{n m}$ are required to be locally supported and their supports are shrinking as level $n$ increases. Denote the support of the function $w_{n m}$ by $S_{n m} . S_{n m}$ is contained in $E_{n i}$ for some $i \in \mathbb{Z}_{e(n)}$.

On the other hand, a set of linear functionals in $\mathbb{V}^{*}$ given by

$$
L_{n}:=\left\{\ell_{n m}: m \in \mathbb{Z}_{w(n)}\right\}, \quad n \in \mathbb{N}_{0} .
$$

is introduced. Where, $\mathbb{V}^{*}$ is the dual space of $\mathbb{V}$, and the linear functional $\ell_{n m}$ is a finite sum of point evaluations

$$
\ell_{n m}=\sum_{s \in \hat{E}_{n m}} c_{s} \delta_{s}
$$

where $c_{s}$ are constants and $\hat{E}_{n m}$ is considered as the "support" of the functional $\ell_{n m}$. $\hat{E}_{n m}$ is a finite subset of distinct points in $E_{n m}$ with its cardinality bounded independent of $n \in \mathbb{N}$. For any $s \in E, \delta_{s}$ denotes the linear functional in $\mathbb{V}^{*}$ defined for $v \in \mathbb{V}$ by the equation $\left\langle\delta_{s}, v\right\rangle=v(s)$. We shall need to evaluate $\delta_{s}$ on functions in $\mathbb{X}$. Therefore, as in [AGS] we take any norm preserving extension of $\delta_{s}$ to $\mathbb{X}$ and use the same notation for the extension. In particular, this extension allows us to evaluate piecewise polynomials on $E$.

The linear functionals and multiscale basis functions are constructed such that, for any $n, n^{\prime} \in \mathbb{N}_{0}$

$$
\left\langle\ell_{n^{\prime} m^{\prime}}, w_{n m}\right\rangle=\delta_{n n^{\prime}} \delta_{m m^{\prime}}, \quad(n, m),\left(n^{\prime}, m^{\prime}\right) \in \mathbb{U}, \quad n \leq n^{\prime},
$$


where $\mathbb{U}:=\left\{(i, j): i \in \mathbb{N}_{0}, j \in \mathbb{Z}_{w(i)}\right\}$ and $\delta_{i i^{\prime}}$ is the Kronecker delta. Moreover, for some positive constant $\gamma$,

$$
\sum_{m \in \mathbb{Z}_{w(n)}}\left|\left\langle\ell_{n^{\prime} m^{\prime}}, w_{n m}\right\rangle\right| \leq \gamma, \quad(n, m),\left(n^{\prime}, m^{\prime}\right) \in \mathbb{U}, \quad n>n^{\prime}
$$

To solve (2.2) using collocation scheme, a vector $\mathbf{u}_{n}:=\left[u_{i j}:(i, j) \in \mathbb{U}_{n}\right]$, where $\mathbb{U}_{n}$ is the set of lattice points in $\mathbb{R}^{2}$ defined as $\left\{(i, j): j \in \mathbb{Z}_{w(i)}, i \in \mathbb{Z}_{n+1}\right\}$, is sought such that the function

$$
u_{n}:=\sum_{(i, j) \in \mathbb{U}_{n}} u_{i j} w_{i j}
$$

in $\mathbb{F}_{n}$ satisfies

$$
\left\langle\ell_{i^{\prime} j^{\prime}}, u_{n}-\mathcal{K} u_{n}\right\rangle=\left\langle l_{i^{\prime} j^{\prime}}, f\right\rangle,\left(i^{\prime}, j^{\prime}\right) \in \mathbb{U}_{n} .
$$

Equivalently, a linear system of equations

$$
\left(\mathbf{E}_{n}-\mathbf{K}_{n}\right) \mathbf{u}_{n}=\mathbf{f}_{n}
$$

is obtained, where

$$
\begin{aligned}
\mathbf{K}_{n} & :=\left[\left\langle\ell_{i^{\prime} j^{\prime}}, \mathcal{K} w_{i j}\right\rangle\right]_{f(n) \times f(n)}, \\
\mathbf{E}_{n} & :=\left[\left\langle\ell_{i^{\prime} j^{\prime}}, w_{i j}\right\rangle\right]_{f(n) \times f(n)},
\end{aligned}
$$

and

$$
\mathbf{f}_{n}:=\left[\left\langle l_{i^{\prime} j^{\prime}}, f\right\rangle:\left(i^{\prime}, j^{\prime}\right) \in \mathbb{U}_{n}\right]
$$

\subsection{General Construction of Multiscale Functions and Functionals}

We start with a positive integer $\mu$ and a family of contractive affine mappings on $\mathbb{R}^{d} \Phi:=$ $\left\{\phi_{e}: e \in Z_{\mu}\right\}$. It is known (see, $[\mathrm{H}]$ ) that there exists a unique compact subset $E \in \mathbb{R}^{d}$ such that

$$
\Phi(E)=E,
$$


where

$$
\Phi(E):=\bigcup_{e \in Z_{\mu}} \phi_{e}(E)
$$

The set $E$ is called the invariant set associated with the family of mappings $\Phi$. Additional restriction to the family of mappings $\Phi$ is made:

(a) For every $e \in \mathbb{Z}_{\mu}, \phi_{e}^{-1}$ is continuous on $E$;

(b) The set $E$ has non-empty interior and

$$
\operatorname{meas}\left(\phi_{e}(E) \cap \phi_{e^{\prime}}(E)\right)=0, \quad e, e^{\prime} \in \mathbb{Z}_{\mu}, e \neq e^{\prime}
$$

$\Phi$ is used to obtain multiscale partitions $\left\{E_{n}: n \in \mathbb{Z}_{0}\right\}$ of the set $E$. To this end, let $\mathbf{e}:=\left(e_{0}, e_{1}, \ldots, e_{n-1}\right) \in \mathbb{Z}_{\mu}^{n}:=\mathbb{Z}_{\mu} \times \ldots \times \mathbb{Z}_{\mu}$. Define a composite mapping

$$
\phi_{\mathbf{e}}:=\phi_{e_{0}} \circ \phi_{e_{1}} \circ \ldots \circ \phi_{e_{n-1}}
$$

and a number

$$
\mu(\mathbf{e}):=\mu^{n-1} e_{0}+\ldots+\mu e_{n-2}+e_{n-1} .
$$

Since $E$ is an invariant set with respect to $\Phi$ and conditions $(a)$ with $(b)$ it follows that the collection of sets

$$
E_{n}:=\left\{E_{n, \mathbf{e}}: E_{n, \mathbf{e}}=\phi_{\mathbf{e}}(E), \mathbf{e} \in \mathbb{Z}_{\mu}^{n}\right\}
$$

form a partition of $E$. Let $\mathbb{F}_{n}$ be the spaces of all functions such that their restriction to any cell $E_{n, \mathbf{e}}, \mathbf{e} \in Z_{\mu}^{n}$ is a polynomial of total degree $\leq k-1, k \geq 1$. Therefore

$$
m:=\operatorname{dim} \mathbb{F}_{0}=\left(\begin{array}{c}
k+d-1 \\
d
\end{array}\right) .
$$

Let

$$
G_{0}=\left\{t_{j}: t_{j} \in E, \text { and } t_{j} \neq t_{i} \text {, for } i \neq j, i, j \in \mathbb{Z}_{m}\right\}
$$

which is refinable relative to the mappings $\Phi$, that is

$$
G_{0} \subset \Phi\left(G_{0}\right)
$$

Set

$$
G_{1}:=\Phi\left(G_{0}\right), \quad V_{1}:=G_{1} \backslash G_{0}=\left\{t_{m+j}: j \in \mathbb{Z}_{r}\right\}
$$


with $r:=(\mu-1) m$. Now, Assume

$$
\mathbb{F}_{0}:=\operatorname{span}\left\{\psi_{j}: j \in \mathbb{Z}_{m}\right\}
$$

and $\psi_{0}, \psi_{1}, \ldots, \psi_{m-1}$ satisfy unique Lagrange interpolation conditions

$$
\psi_{i}\left(t_{j}\right)=\delta_{i j}, \quad i, j \in \mathbb{Z}_{m}
$$

A construction of refinable points $\left\{t_{j}: j \in \mathbb{Z}_{m}\right\} \in E$ that admits a unique $d$-dimensional Lagrange interpolation is presented in [MSX1]. Introduce linear operators $\mathcal{T}_{e}: \mathbb{X} \rightarrow \mathbb{X}, e \in$ $\mathbb{Z}_{\mu}$ defined by

$$
\left(T_{e} x\right)(t):=x\left(\phi_{e}^{-1}(t)\right) \chi_{\phi_{e}(E)}(t)
$$

where $\chi_{S}$ denote the characteristic function of the set $S$. Therefore it follows that

$$
\mathbb{F}_{n}=\bigoplus_{e \in Z_{\mu}} \mathcal{T}_{e} \mathbb{F}_{n-1}, \quad n \in \mathbb{Z}
$$

where $\mathbb{A} \oplus \mathbb{B}$ denotes the direct sum of the spaces $\mathbb{A}$ and $\mathbb{B}$. The functions $\psi_{m+j} \in \mathbb{F}_{1}$ such that $\psi_{m+j}\left(t_{m+i}\right)=\delta_{i j}, t_{m+i} \in V_{1}, i, j \in \mathbb{Z}_{r}$ with $\psi_{j} \in \mathbb{F}_{0}, j \in \mathbb{Z}_{m}$, defined in (2.33) form a basis for $\mathbb{F}_{1}$. This basis doesn't have vanishing moment. So instead another basis for $\mathbb{F}_{1}$ consisting of functions with vanishing moments is constructed. For this purpose, set

$$
w_{0 j}:=\psi_{j}, \quad j \in \mathbb{Z}_{m}
$$

let $q:=m+r$ and for $j \in \mathbb{Z}_{r}$ find a vector $\left[c_{j s}: s \in \mathbb{Z}_{q}\right] \in \mathbb{R}^{q}$ such that

$$
w_{1 j}:=\sum_{s \in \mathbb{Z}_{q}} c_{j s} \psi_{s}, \quad j \in \mathbb{Z}_{r}
$$

satisfies

$$
\left(w_{1 j}, w_{0 j^{\prime}}\right)=0, \quad j^{\prime} \in \mathbb{Z}_{m}, j \in \mathbb{Z}_{r},
$$

where (, ) is the usual $L^{2}$ inner product of two functions on $E$. (2.38) leads to a linear system of rank $m$ with $m$ equations and $q$ unknowns, thus it has $r$ linearly independent solutions. Denote them by $w_{1 j}, j \in \mathbb{Z}_{r}$. These functions form a basis for the space $\mathbb{W}_{1}$. To construct a basis for $\mathbb{W}_{i}, i \geq 2$, for $\mathbf{e}:=\left(e_{0}, \ldots, e_{n-1}\right) \in \mathbb{Z}_{\mu}^{n}$ introduce a composite operator $\mathcal{T}_{\mathbf{e}}$ by

$$
\mathcal{T}_{\mathrm{e}}:=\mathcal{T}_{e_{0}} \circ \ldots \circ \mathcal{T}_{e_{n-1}}
$$


For $i=2,3, \ldots, n$, let

$$
w_{i j}:=\mathcal{T}_{\mathbf{e}} w_{1 l}, \quad j=\mu(\mathbf{e}) r+l, \mathbf{e} \in \mathbb{Z}_{\mu}^{i-1}, l \in \mathbb{Z}_{r}
$$

and

$$
\mathbb{W}_{i}:=\operatorname{span}\left\{w_{i j}: j \in \mathbb{Z}_{w(i)}\right\}
$$

Note that the support of $w_{i j}$ is contained in $S_{i j}:=\phi_{\mathbf{e}}(E), j \in \mathbb{Z}_{w(i)}$.

The collocation functionals are defined in a similar manner. Define

$$
\ell_{0 j}:=\delta_{t_{j}}, \quad j \in \mathbb{Z}_{m}
$$

and for $j^{\prime} \in \mathbb{Z}_{r}$, find the vector $\left[c_{j^{\prime} s}^{\prime}: s \in \mathbb{Z}_{q}\right]$ such that

$$
\ell_{1 j^{\prime}}:=\sum_{s \in \mathbb{Z}_{q}} c_{j^{\prime} s}^{\prime} \delta_{t_{s}}, \quad j^{\prime} \in \mathbb{Z}_{r}
$$

satisfies equations

$$
\left\langle\ell_{1 j^{\prime}}, w_{0 j}\right\rangle=0, \quad j \in \mathbb{Z}_{m}, \quad j^{\prime} \in \mathbb{Z}_{r}
$$

and

$$
\left\langle\ell_{1 j^{\prime}}, w_{1 j}\right\rangle=\delta_{j j^{\prime}}, \quad j \in \mathbb{Z}_{r}, \quad j^{\prime} \in \mathbb{Z}_{r}
$$

(2.44) and (2.45) lead to a $q \times q$ linear system of equations. The coefficient matrix for this linear system of equations is

$$
\mathbf{A}:=\left[\left\langle\delta_{t_{i^{\prime} j^{\prime}}}, w_{i j}\right\rangle\right]_{(i, j),\left(i^{\prime} j^{\prime}\right) \in \mathbb{U}_{1}} .
$$

A is proved to be nonsingular in [CMX3]. To generate a multiscale collocation functionals, introduce for any $e \in \mathbb{Z}_{\mu}$ a linear operator $\mathcal{L}_{e}: V^{*} \rightarrow V^{*}$ defined by the equation

$$
\left\langle\mathcal{L}_{e} \ell, v\right\rangle=\left\langle\ell, v \circ \phi_{e}\right\rangle, \quad v \in \mathbb{X}, \ell \in \mathbb{X}^{*}
$$

Moreover, for $\mathbf{e}:=\left(e_{0}, \ldots, e_{n-1}\right) \in \mathbb{Z}_{\mu}^{n}$, define a composite operator

$$
\mathcal{L}_{\mathrm{e}}:=\mathcal{L}_{e_{0}} \circ \ldots \circ \mathcal{L}_{e_{n-1}}
$$

Consequently, for any $\mathbf{e}, \mathbf{e}^{\prime} \in \mathbb{Z}_{\mu}^{i}, w \in \mathbb{X}$ and $\ell \in \mathbb{X}^{*}$, we have that

$$
\left\langle\mathcal{L}_{\mathbf{e}} l, \mathcal{T}_{\mathbf{e}^{\prime}} w\right\rangle=\langle l, w\rangle \delta_{\mathbf{e e}^{\prime}}
$$


In addition, for $i>1, j=\mu(\mathbf{e}) r+l, \mathbf{e} \in \mathbb{Z}_{\mu}^{i-1}, l \in \mathbb{Z}_{r}$ we define

$$
\ell_{i j}:=\mathcal{L}_{\mathbf{e}} \ell_{1 l}
$$

Note that

$$
\left\langle\ell_{i j}, v\right\rangle=\left\langle\ell_{1 l}, v \circ \phi_{\mathbf{e}}\right\rangle=\sum_{s \in \mathbb{Z}_{q}} c^{\prime}{ }_{l s} v\left(\phi_{\mathbf{e}}\left(t_{s}\right)\right) .
$$

Observe that the "support" of $\ell_{i j}$ is also contained in $S_{i j}$. 


\section{Chapter 3}

\section{Wavelets and Collocation Functionals on the Unit Triangle}

Our following discussion is on the unit triangle $\Omega:=\{(x, y) \mid 0 \leq x \leq 1,0 \leq y \leq 1-x\}$. We first introduce a family of contractive mappings $\Phi=\left\{\phi_{e}, e \in \mathbb{Z}_{4}\right\}$ to subdivide the domain $\Omega$. Define

$$
\begin{aligned}
\phi_{0}(x, y) & =\left(\frac{x}{2}, \frac{y}{2}\right), \\
\phi_{1}(x, y) & =\left(\frac{x+1}{2}, \frac{y}{2}\right), \\
\phi_{2}(x, y) & =\left(\frac{x}{2}, \frac{y+1}{2}\right) . \\
\phi_{3}(x, y) & =\left(\frac{1-x}{2}, \frac{1-y}{2}\right) .
\end{aligned}
$$

These four mappings subdivide the unit triangle $\Omega$ into four small triangles $\Omega_{0}, \Omega_{1}, \Omega_{2}$, and $\Omega_{3}$ (see Fig. 3.1), with $\Omega_{i}=\phi_{i}(\Omega), i \in \mathbb{Z}_{4}$. We then can define four operators $\mathcal{T}_{i}, i \in \mathbb{Z}_{4}$ through the four mappings $\phi_{i}, i \in \mathbb{Z}_{4}$, namely,

$$
\left(\mathcal{T}_{i} \circ g\right)(\xi, \eta)=g\left(\phi_{i}^{-1}(\xi, \eta)\right) \chi_{\Omega_{i}}(\xi, \eta), \quad i \in \mathbb{Z}_{4}
$$


Yi Wang Chapter 3. Wavelets and collocation functionals on the Unit Triangle

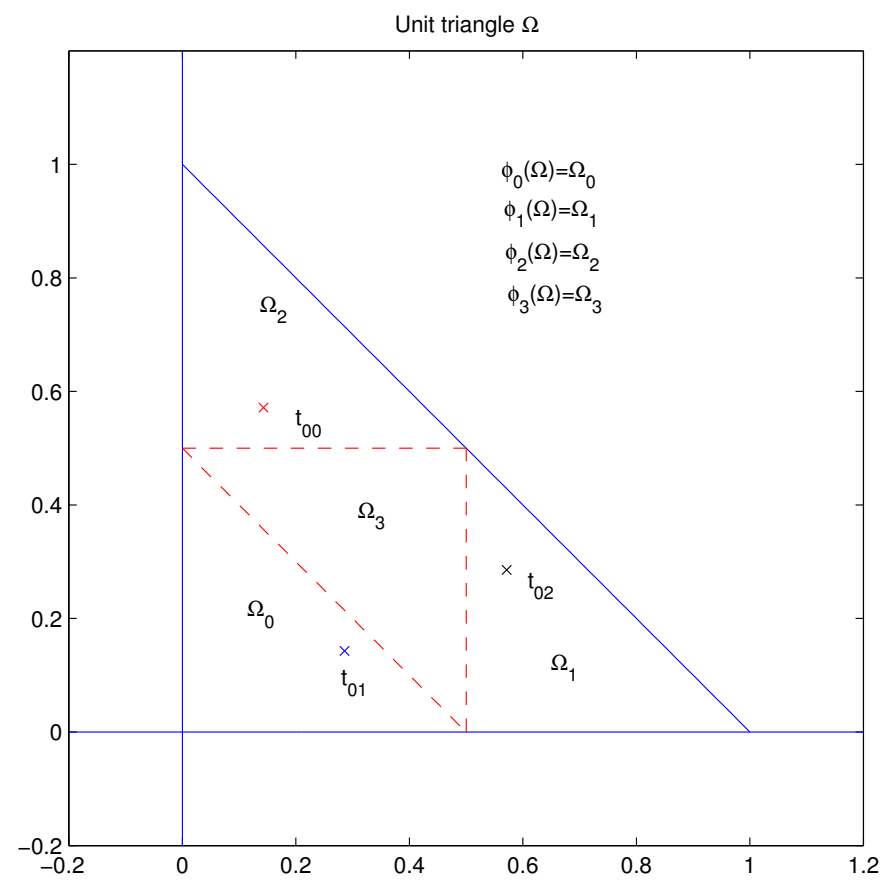

Figure 3.1: Original domain-unit triangle $\Omega$

where,

$$
\begin{aligned}
\phi_{0}^{-1}(x, y) & =(2 x, 2 y) \\
\phi_{1}^{-1}(x, y) & =(2 x-1,2 y) \\
\phi_{2}^{-1}(x, y) & =(2 x, 2 y-1) \\
\phi_{3}^{-1}(x, y) & =(1-2 x, 1-2 y) .
\end{aligned}
$$

We choose as the original collocation points $t_{00}=\left(\frac{1}{7}, \frac{4}{7}\right), t_{01}=\left(\frac{2}{7}, \frac{1}{7}\right)$, and $t_{02}=\left(\frac{4}{7}, \frac{2}{7}\right)$ (see Fig. 3.1). Note the set $G_{0}:=\left\{t_{0 j}: j \in \mathbb{Z}_{3}\right\}$ is refinable with respect to the family of mappings $\Phi:=\left\{\phi_{e}: e \in \mathbb{Z}_{4}\right\}$. 


\subsection{The Approximation Spaces}

Let $\mathbb{W}_{0}$ be a subspace of $\mathbb{X}$ with $\operatorname{dim} \mathbb{W}_{0}=3$. Assume $\mathbb{W}_{0}$ is a space of linear polynomials on $\Omega$. Moreover assume

$$
\mathbb{W}_{0}=\operatorname{span}\left\{w_{0 j}, j \in \mathbb{Z}_{3}\right\}
$$

where, $w_{0 j}, j \in \mathbb{Z}_{3}$ are determined by interpolating conditions

$$
w_{0 j}\left(t_{0 k}\right)=\delta_{j k}, \quad j, k \in \mathbb{Z}_{3} .
$$

To find $w_{0 j}, j \in \mathbb{Z}_{3}$, write $w_{0 j}=b_{j_{0}} x+b_{j_{1}} y+b_{j_{2}}, j \in \mathbb{Z}_{3}$. Define a matrix $\mathbf{B}=\left[b_{j k}\right]: j, k \in$ $\mathbb{Z}_{3}, \mathbf{B} \in M_{3,3}$ (throughout this thesis $M_{m, n}$ denotes a $m \times n$ matrix over the field $\mathbb{R}$ ). Let

$$
\mathbf{A}=\left[\begin{array}{ccc}
\frac{1}{7} & \frac{4}{7} & 1 \\
\frac{2}{7} & \frac{1}{7} & 1 \\
\frac{4}{7} & \frac{2}{7} & 1
\end{array}\right]
$$

Solve

$$
\mathrm{AB}=\mathbf{I}
$$

for $\mathbf{B}$, where, $\mathbf{I}$ is the identity matrix in $M_{3,3}$. We obtain

$$
\mathbf{B}=\left[\begin{array}{ccc}
-1 & -2 & 3 \\
2 & -3 & 1 \\
0 & 2 & -1
\end{array}\right]
$$

Thus,

$$
\begin{aligned}
& w_{00}(x, y)=-x+2 y, \\
& w_{01}(x, y)=-2 x-3 y+2, \\
& w_{02}(x, y)=3 x+y-1 .
\end{aligned}
$$

With $\mathbb{W}_{0}\left(=\mathbb{F}_{0}\right)$, we can find

$$
\mathbb{F}_{1}=\bigoplus_{j \in \mathbb{Z}_{4}} \mathcal{T}_{j} \mathbb{W}_{0}
$$

Let $\mathbb{W}_{1}$ be the orthogonal complement of $\mathbb{W}_{0}$ in $\mathbb{F}_{1}$, and define

$$
\mathbb{W}_{n}=\bigoplus_{j \in \mathbb{Z}_{4}} \mathcal{T}_{j} \mathbb{W}_{n-1}, n \geq 2, n \in \mathbb{Z}
$$


Yi Wang Chapter 3. Wavelets and collocation functionals on the Unit Triangle

We can easily find that

$$
\operatorname{Dim}_{n}=3 \cdot 4^{n}, n \in \mathbb{N}_{0},
$$

and

$$
\operatorname{Dim} \mathbb{W}_{n}=3^{2} \cdot 4^{n-1}, n \geq 1, n \in \mathbb{Z}
$$

\subsection{Initial Wavelets in $\mathbb{W}_{1}$}

We proceed in this section to construct a wavelet basis having vanishing moment of $\mathbb{W}_{1}$. Now,

$$
\begin{aligned}
& \mathcal{T}_{0} \circ w_{00}=(-2 x+4 y) \chi_{\Omega_{0}}, \\
& \mathcal{T}_{0} \circ w_{01}=(-4 x-6 y+2) \chi_{\Omega_{0}}, \\
& \mathcal{T}_{0} \circ w_{02}=(6 x+2 y-1) \chi_{\Omega_{0}}, \\
& \mathcal{T}_{1} \circ w_{00}=(-2 x+4 y+1) \chi_{\Omega_{1}}, \\
& \mathcal{T}_{1} \circ w_{01}=(-4 x-6 y+4) \chi_{\Omega_{1}}, \\
& \mathcal{T}_{1} \circ w_{02}=(6 x+2 y-4) \chi_{\Omega_{1}}, \\
& \mathcal{T}_{2} \circ w_{00}=(-2 x+4 y-2) \chi_{\Omega_{2}}, \\
& \mathcal{T}_{2} \circ w_{01}=(-4 x-6 y+5) \chi_{\Omega_{2}}, \\
& \mathcal{T}_{2} \circ w_{02}=(6 x+2 y-2) \chi_{\Omega_{2}}, \\
& \mathcal{T}_{3} \circ w_{00}=(2 x-4 y+1) \chi_{\Omega_{3}}, \\
& \mathcal{T}_{3} \circ w_{01}=(4 x+6 y-3) \chi_{\Omega_{3}}, \\
& \mathcal{T}_{3} \circ w_{02}=(-6 x-2 y+3) \chi_{\Omega_{3}} .
\end{aligned}
$$

Note $\mathcal{T}_{0} w_{02}\left(t_{01}\right)=\mathcal{T}_{1} w_{00}\left(t_{02}\right)=\mathcal{T}_{2} w_{01}\left(t_{00}\right)=1$. Write

$$
\begin{aligned}
\mathbf{S}= & {\left[w_{00}, w_{01}, w_{02}, \mathcal{T}_{0} w_{00}, \mathcal{T}_{0} w_{01}, \mathcal{T}_{1} w_{01}, \mathcal{T}_{1} w_{02}, \mathcal{T}_{2} w_{00}, \mathcal{T}_{2} w_{02},\right.} \\
& \left.\mathcal{T}_{3} w_{00}, \mathcal{T}_{3} w_{01}, \mathcal{T}_{3} w_{02}\right]^{T} \\
:= & {\left[\psi_{0}, \psi_{1}, \psi_{2}, \psi_{3}, \psi_{4}, \psi_{5}, \psi_{6}, \psi_{7}, \psi_{8}, \psi_{9}, \psi_{10}, \psi_{11}\right]^{T} . }
\end{aligned}
$$


Define the wavelet basis functions of $\mathbb{W}_{1}$ by $w_{1 j}, j \in \mathbb{Z}_{9}$. They are supported on $\Omega$. Let $S:=\left\{\psi_{j}, j \in \mathbb{Z}_{12}\right\}$. Since $S$ is a set of basis functions of $\mathbb{F}_{1}$, we have

$$
w_{1 j}=\sum_{s \in \mathbb{Z}_{12}} c_{j s} \psi_{s}
$$

To make $w_{1 j}, j \in \mathbb{Z}_{9}$ have vanishing moment, require that

$$
\left(w_{1 k}, x^{i} y^{j}\right)=0 \quad k \in \mathbb{Z}_{9}
$$

where, $i, j \in \mathbb{Z}_{2}, i+j \leq 1$. Observe that

$$
\begin{aligned}
\int_{\Omega_{0}} f(x, y) \mathrm{d} S & =\int_{0}^{\frac{1}{2}} \int_{0}^{-x+\frac{1}{2}} f(x, y) \mathrm{d} y \mathrm{~d} x, \\
\int_{\Omega_{1}} f(x, y) \mathrm{d} S & =\int_{\frac{1}{2}}^{1} \int_{0}^{-x+1} f(x, y) \mathrm{d} y \mathrm{~d} x \\
\int_{\Omega_{2}} f(x, y) \mathrm{d} S & =\int_{0}^{\frac{1}{2}} \int_{\frac{1}{2}}^{-x+1} f(x, y) \mathrm{d} y \mathrm{~d} x, \\
\int_{\Omega_{3}} f(x, y) \mathrm{d} S & =\int_{0}^{\frac{1}{2}} \int_{-x+\frac{1}{2}}^{\frac{1}{2}} f(x, y) \mathrm{d} y \mathrm{~d} x .
\end{aligned}
$$

(3.17) yields the following linear system

$$
\mathrm{Ac}=0,
$$

where,

$$
\mathbf{A}=\left[\begin{array}{cccccccccccc}
\frac{1}{6} & \frac{1}{6} & \frac{1}{6} & \frac{1}{24} & \frac{1}{24} & \frac{1}{24} & \frac{1}{24} & \frac{1}{24} & \frac{1}{24} & \frac{1}{24} & \frac{1}{24} & \frac{1}{24} \\
0 & \frac{1}{24} & \frac{1}{8} & 0 & \frac{1}{192} & \frac{5}{192} & \frac{7}{192} & 0 & \frac{1}{64} & \frac{1}{48} & \frac{1}{64} & \frac{1}{192} \\
\frac{1}{8} & 0 & \frac{1}{24} & \frac{1}{64} & 0 & 0 & \frac{1}{192} & \frac{7}{192} & \frac{5}{192} & \frac{1}{192} & \frac{1}{48} & \frac{1}{64}
\end{array}\right]
$$

and

$$
\mathbf{c}=\left[\begin{array}{llllllllllll}
c_{0} & c_{1} & c_{2} & c_{3} & c_{4} & c_{5} & c_{6} & c_{7} & c_{8} & c_{9} & c_{10} & c_{11}
\end{array}\right]^{T} .
$$

The null space of above linear system has dimension equal to 9 , and is spanned by the set $\left\{\mathbf{w}_{i}: i \in \mathbb{Z}_{9}\right\}$, where $\mathbf{w}_{i}$ corresponds to the $i$ th row vector of the following $9 \times 12$ matrix 
$\mathbf{C}:=\left[c_{j k}: j, k \in \mathbb{Z}_{12}\right]$

$$
\mathbf{C}=\left[\begin{array}{cccccccccccc}
-\frac{1}{8} & -\frac{1}{8} & 0 & 0 & 0 & 0 & 0 & 0 & 0 & 0 & 0 & 1 \\
-\frac{1}{8} & 0 & -\frac{1}{8} & 0 & 0 & 0 & 0 & 0 & 0 & 0 & 1 & 0 \\
0 & -\frac{1}{8} & -\frac{1}{8} & 0 & 0 & 0 & 0 & 0 & 0 & 1 & 0 & 0 \\
-\frac{9}{56} & \frac{3}{56} & -\frac{1}{7} & 0 & 0 & 0 & 0 & 0 & 1 & 0 & 0 & 0 \\
-\frac{2}{7} & \frac{3}{56} & -\frac{1}{56} & 0 & 0 & 0 & 0 & 1 & 0 & 0 & 0 & 0 \\
\frac{3}{56} & -\frac{1}{56} & -\frac{2}{7} & 0 & 0 & 0 & 1 & 0 & 0 & 0 & 0 & 0 \\
\frac{3}{56} & -\frac{1}{7} & -\frac{9}{56} & 0 & 0 & 1 & 0 & 0 & 0 & 0 & 0 & 0 \\
-\frac{1}{56} & -\frac{2}{7} & \frac{3}{56} & 0 & 1 & 0 & 0 & 0 & 0 & 0 & 0 & 0 \\
-\frac{1}{7} & -\frac{9}{56} & \frac{3}{56} & 1 & 0 & 0 & 0 & 0 & 0 & 0 & 0 & 0
\end{array}\right]
$$

Now we can use matrix form to express the nine wavelet basis functions in the space $\mathbb{W}_{1}$.

Let $\mathbf{W}$ be the vector of the nine wavelets, i.e.,

$$
\mathbf{W}=\left[w_{10}, w_{11}, w_{12}, w_{13}, w_{14}, w_{15}, w_{16}, w_{17}, w_{18}\right]^{T} .
$$

For any nonsingular matrix $\mathbf{D} \in M_{9,9}$,

$$
\mathbf{W}=\mathbf{D} \cdot \mathrm{C} \cdot \mathrm{S}
$$

is a suitable basis for $\mathbb{W}_{1}$ satisfying (3.17). viz,

$$
w_{1 i}=\sum_{j \in Z_{9}} d_{i j} \sum_{k \in \mathbb{Z}_{12}} c_{j k} \psi_{k} .
$$

We can take $\mathbf{D}=\operatorname{diag}(1,1,1,1,1,1,1,1,1)$ and obtain

$$
\begin{aligned}
& w_{10}(x, y)=\frac{1}{8}(-2+3 x+y)\left(\chi_{\Omega_{0}}+\chi_{\Omega_{1}}+\chi_{\Omega_{2}}\right)+\frac{1}{8}(22-45 x-15 y) \chi_{\Omega_{3}}, \\
& w_{11}(x, y)=\frac{1}{8}(1-2 x-3 y)\left(\chi_{\Omega_{0}}+\chi_{\Omega_{1}}+\chi_{\Omega_{2}}\right)+\frac{1}{8}(-23+30 x+45 y) \chi_{\Omega_{3}}, \\
& w_{12}(x, y)=\frac{1}{8}(-1-x+2 y)\left(\chi_{\Omega_{0}}+\chi_{\Omega_{1}}+\chi_{\Omega_{2}}\right)+\frac{1}{8}(7+15 x-30 y) \chi_{\Omega_{3}}, \\
& w_{13}(x, y)=\frac{1}{8}(2-3 x-5 y)\left(\chi_{\Omega_{0}}+\chi_{\Omega_{1}}+\chi_{\Omega_{3}}\right)+\frac{1}{8}(-14+45 x+11 y) \chi_{\Omega_{2}}, \\
& w_{14}(x, y)=\frac{1}{8}(1+x-6 y)\left(\chi_{\Omega_{0}}+\chi_{\Omega_{1}}+\chi_{\Omega_{3}}\right)+\frac{1}{8}(-15-15 x+26 y) \chi_{\Omega_{2}}, \\
& w_{15}(x, y)=\frac{1}{8}(2-7 x-y)\left(\chi_{\Omega_{0}}+\chi_{\Omega_{2}}+\chi_{\Omega_{3}}\right)+\frac{1}{8}(-30+41 x+15 y) \chi_{\Omega_{1}}, \\
& w_{16}(x, y)=\frac{1}{8}(-1-2 x+3 y)\left(\chi_{\Omega_{0}}+\chi_{\Omega_{2}}+\chi_{\Omega_{3}}\right)+\frac{1}{8}(31-34 x-45 y) \chi_{\Omega_{1}}, \\
& w_{17}(x, y)=\frac{1}{8}(-5+6 x+7 y)\left(\chi_{\Omega_{1}}+\chi_{\Omega_{2}}+\chi_{\Omega_{3}}\right)+\frac{1}{8}(11-26 x-41 y) \chi_{\Omega_{0}}, \\
& w_{18}(x, y)=\frac{1}{8}(-3+5 x+2 y)\left(\chi_{\Omega_{1}}+\chi_{\Omega_{2}}+\chi_{\Omega_{3}}\right)+\frac{1}{8}(-3-11 x+34 y) \chi_{\Omega_{0}}
\end{aligned}
$$

The pictures of $w_{0 j}, j \in \mathbb{Z}_{3}$ and $w_{1 k}, k \in \mathbb{Z}_{9}$ are shown in Fig. 3.2 through Fig. 3.13. 


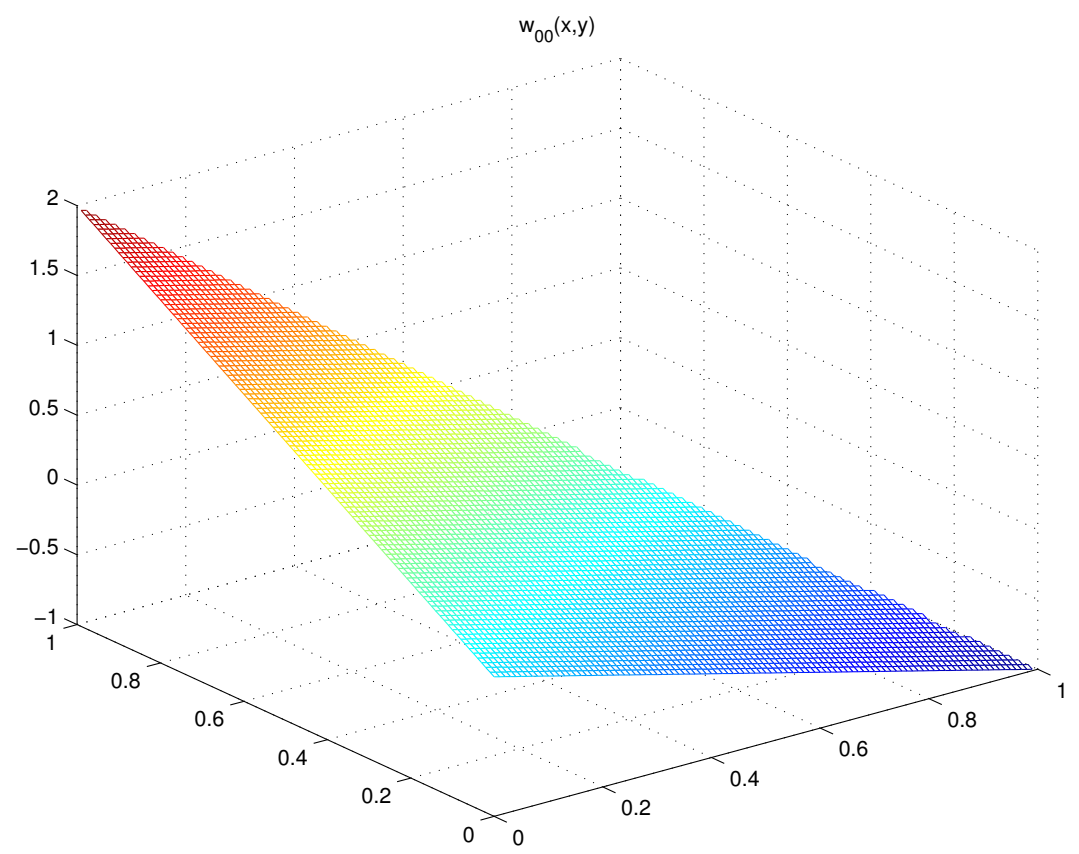

Figure 3.2: Scaling function $w_{00}$

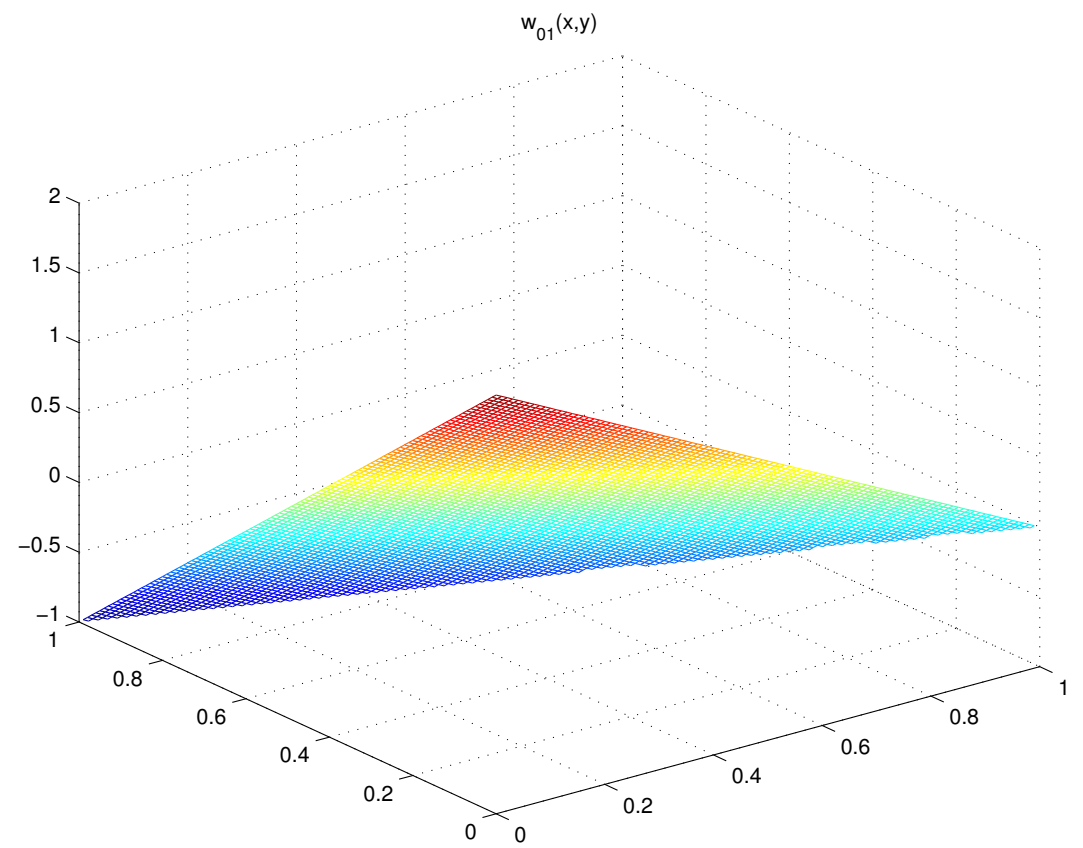

Figure 3.3: Scaling function $w_{01}$ 


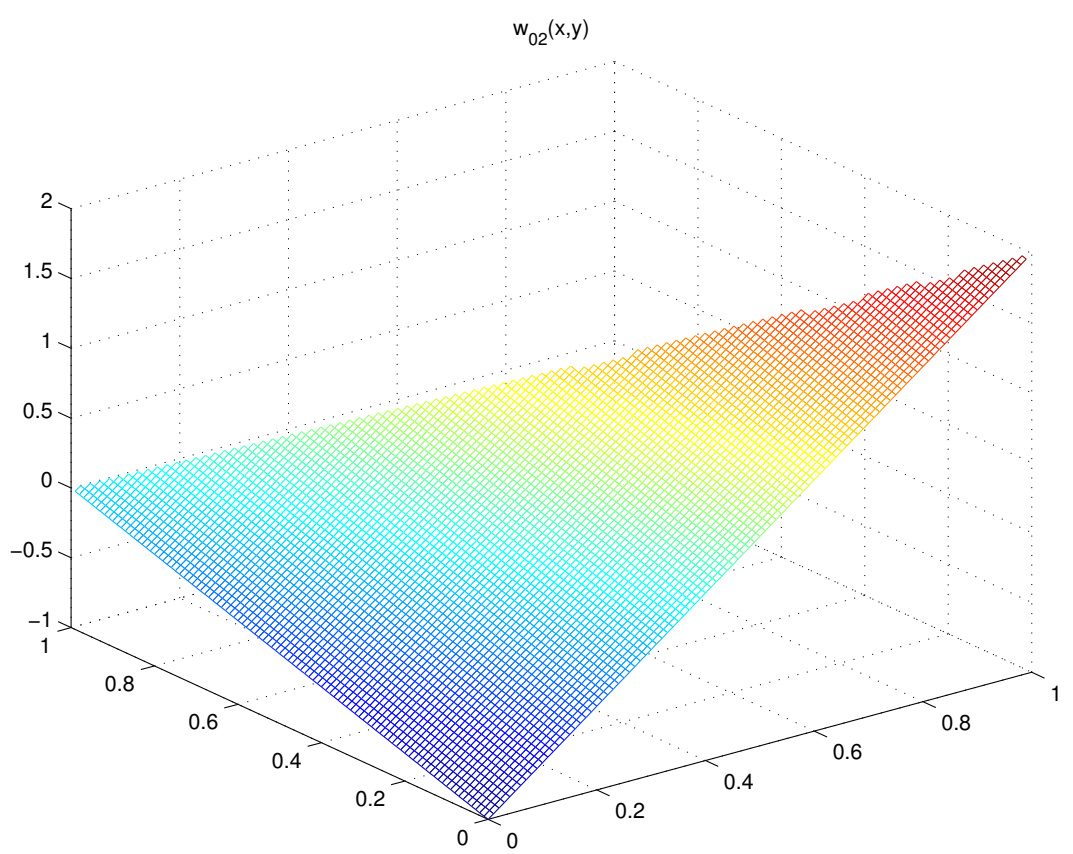

Figure 3.4: Scaling function $w_{02}$

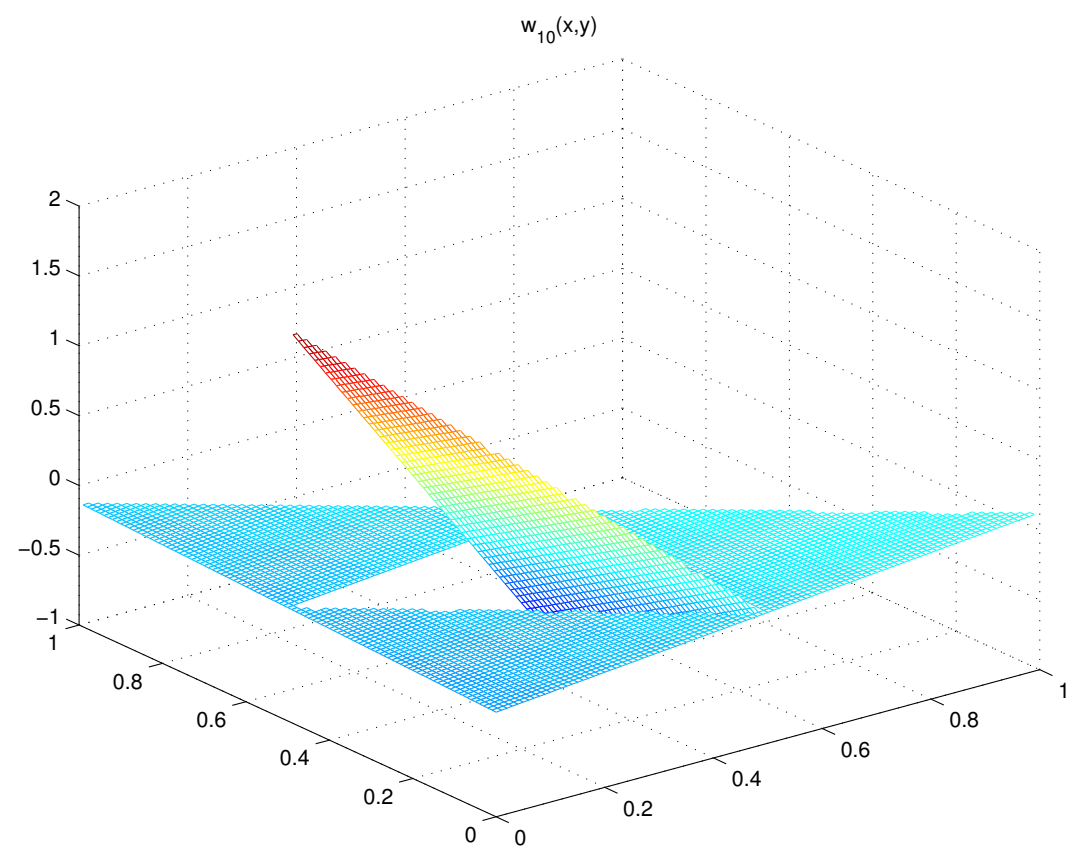

Figure 3.5: Wavelet function $w_{10}$ 


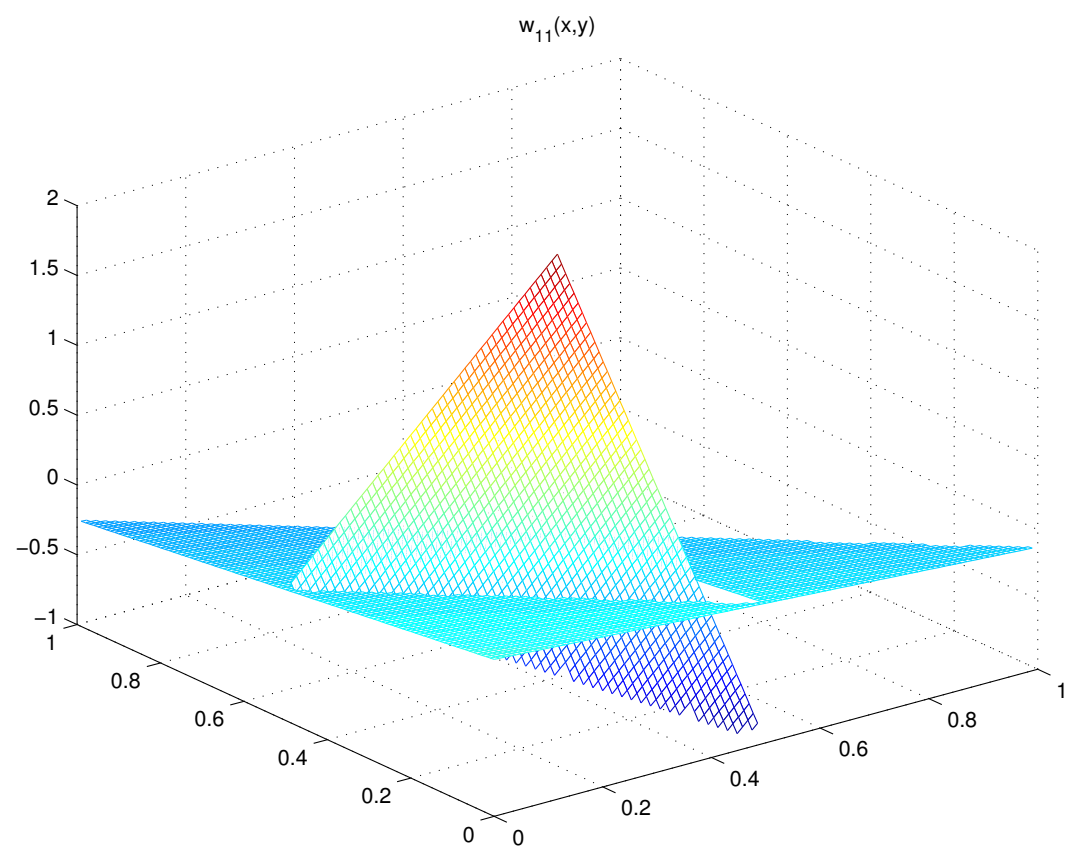

Figure 3.6: Wavelet function $w_{11}$

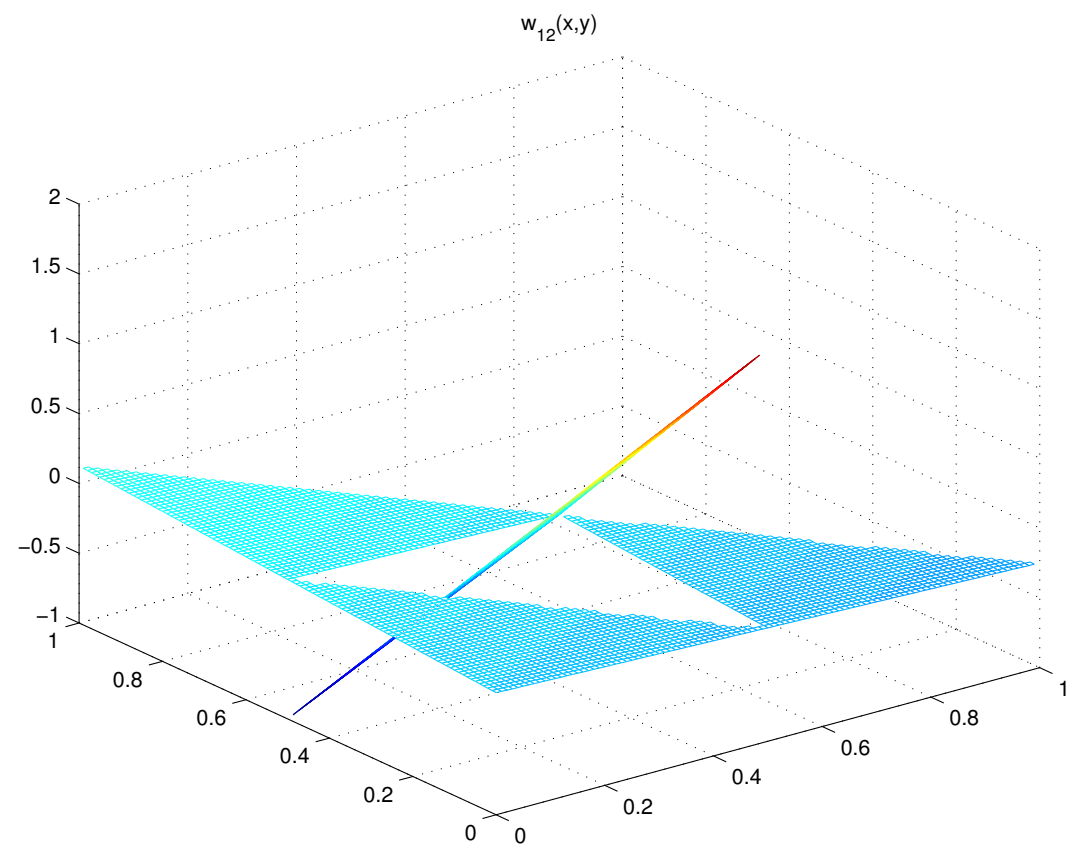

Figure 3.7: Wavelet function $w_{12}$ 


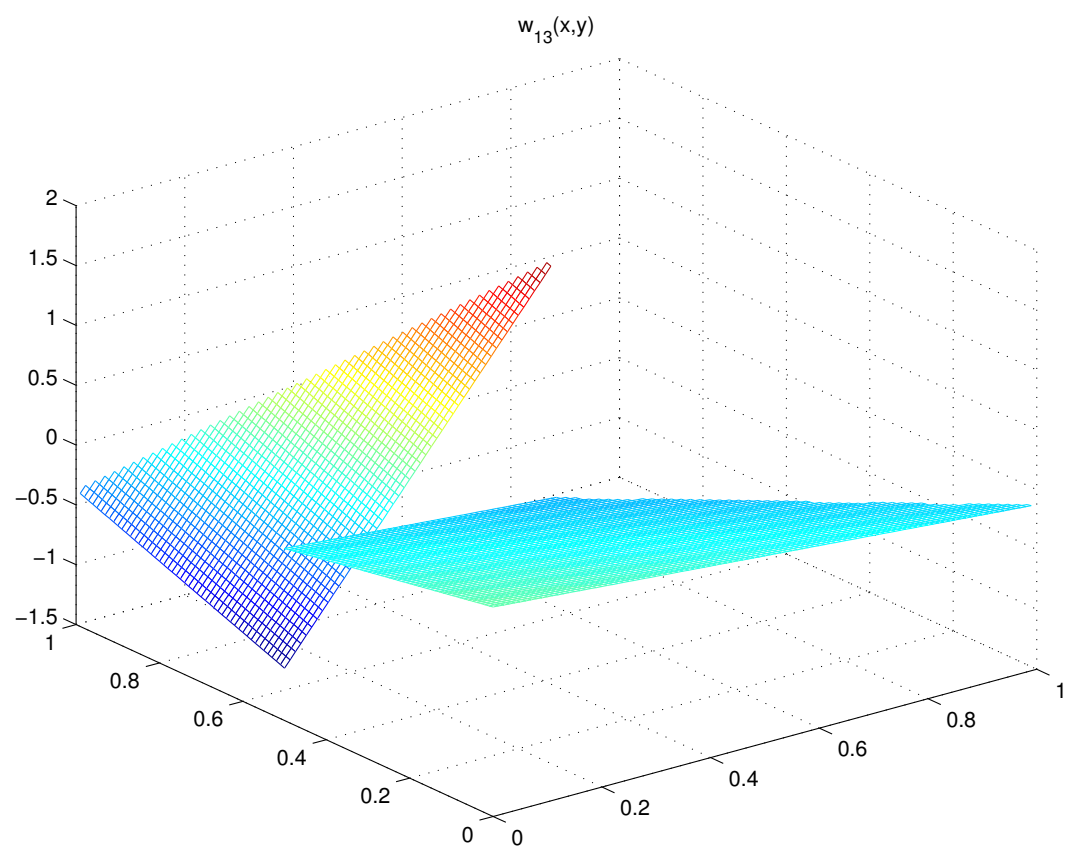

Figure 3.8: Wavelet function $w_{13}$

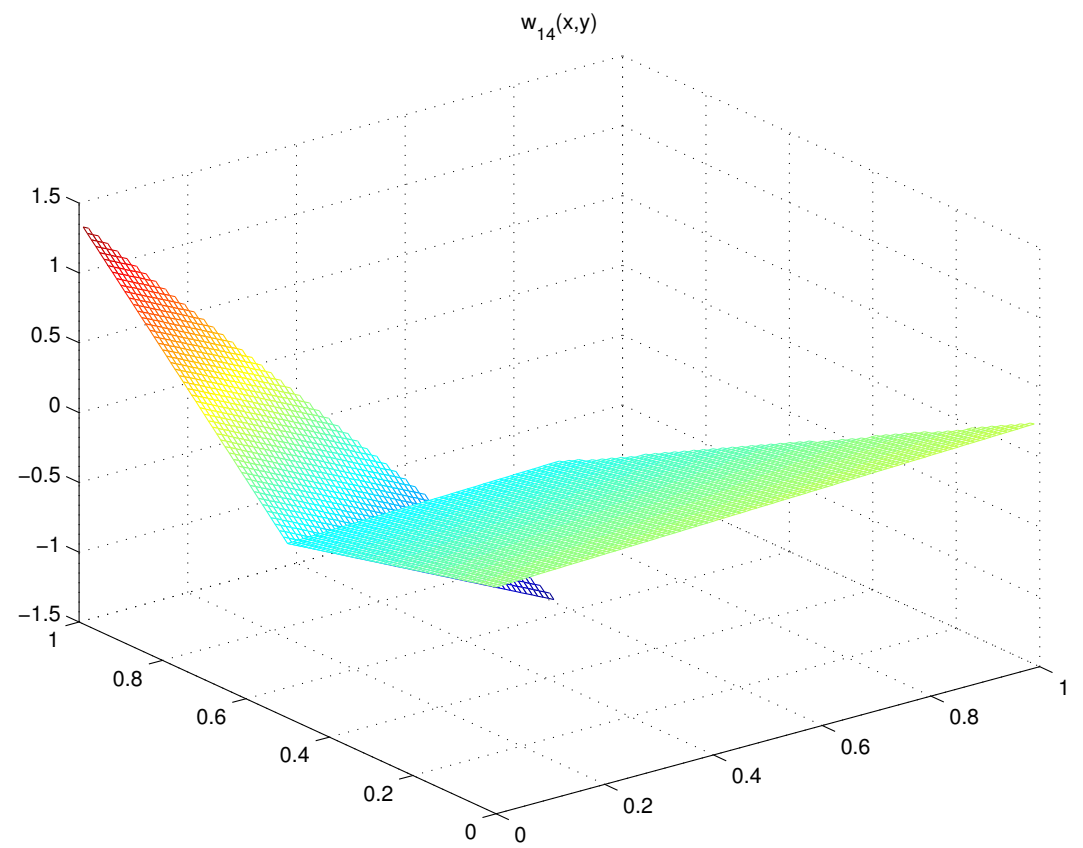

Figure 3.9: Wavelet function $w_{14}$ 


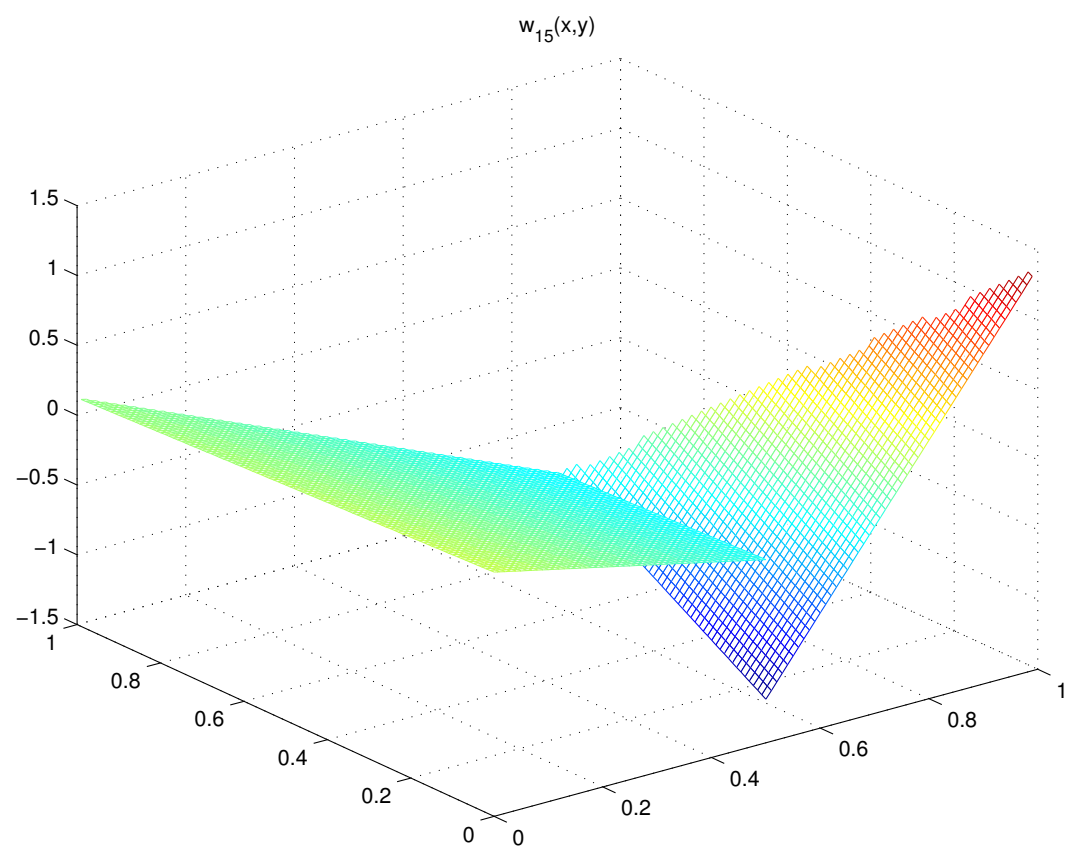

Figure 3.10: Wavelet function $w_{15}$

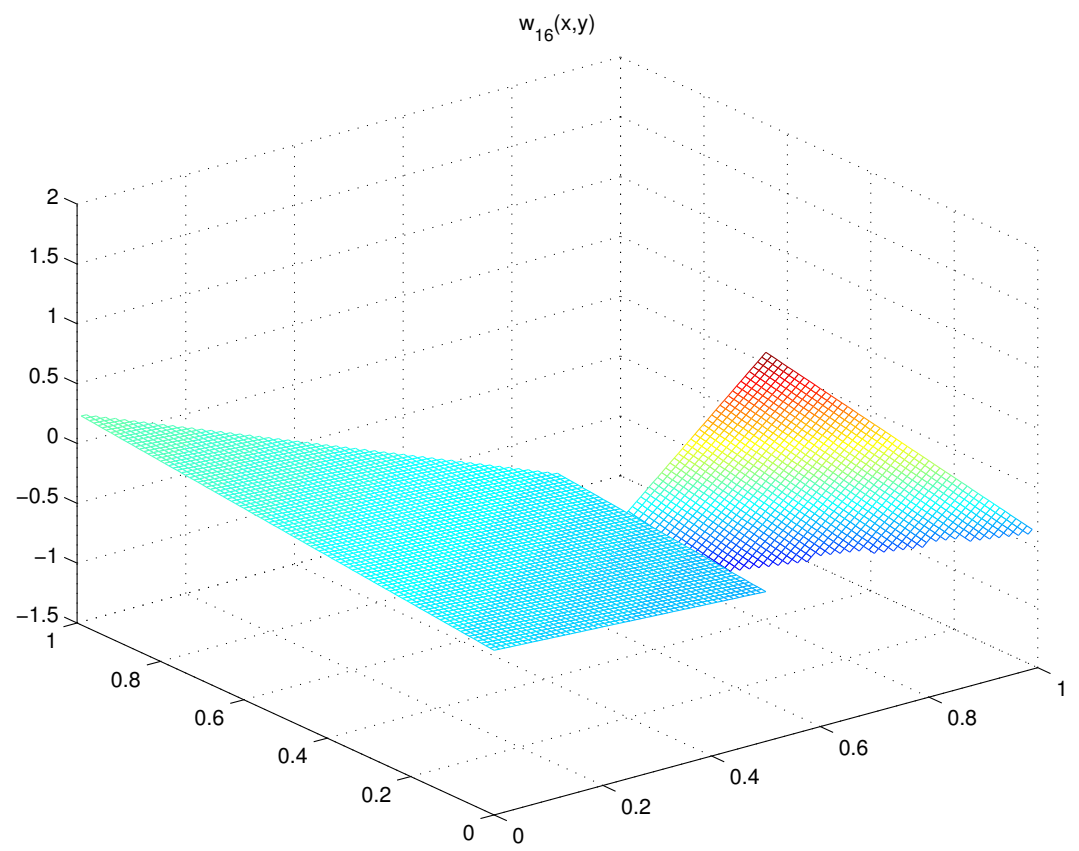

Figure 3.11: Wavelet function $w_{16}$ 


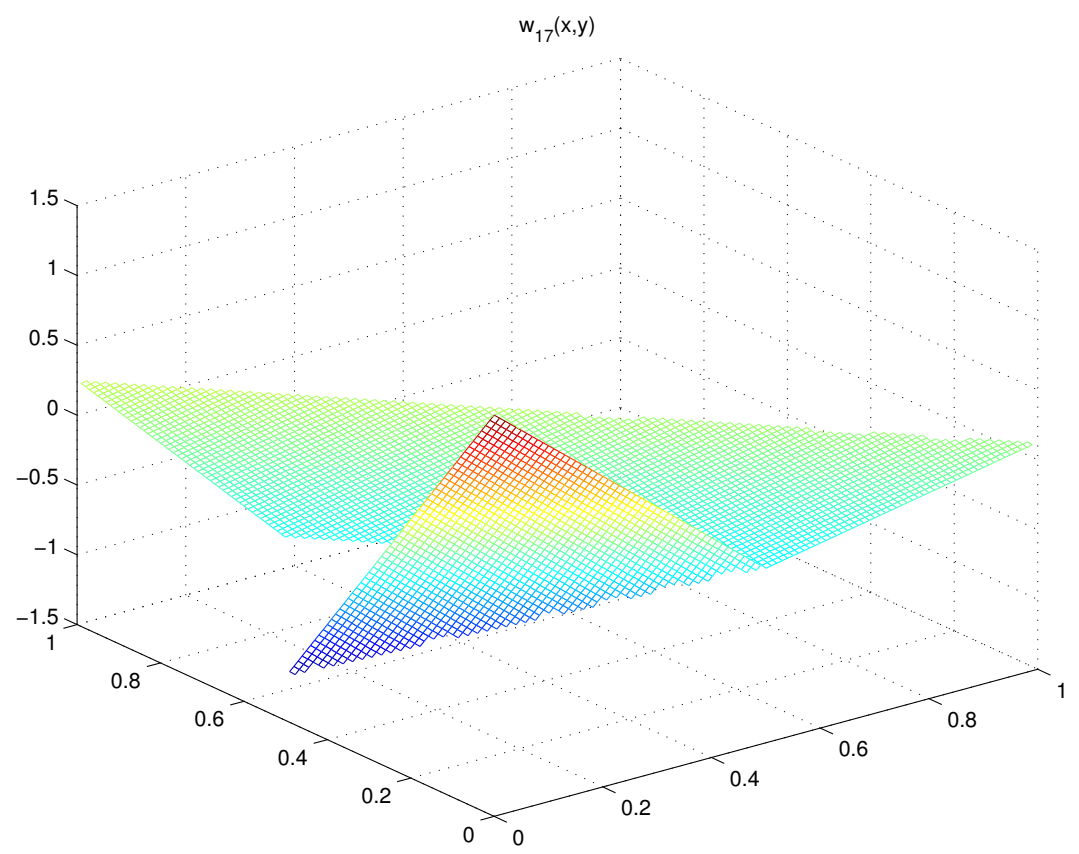

Figure 3.12: Wavelet function $w_{17}$

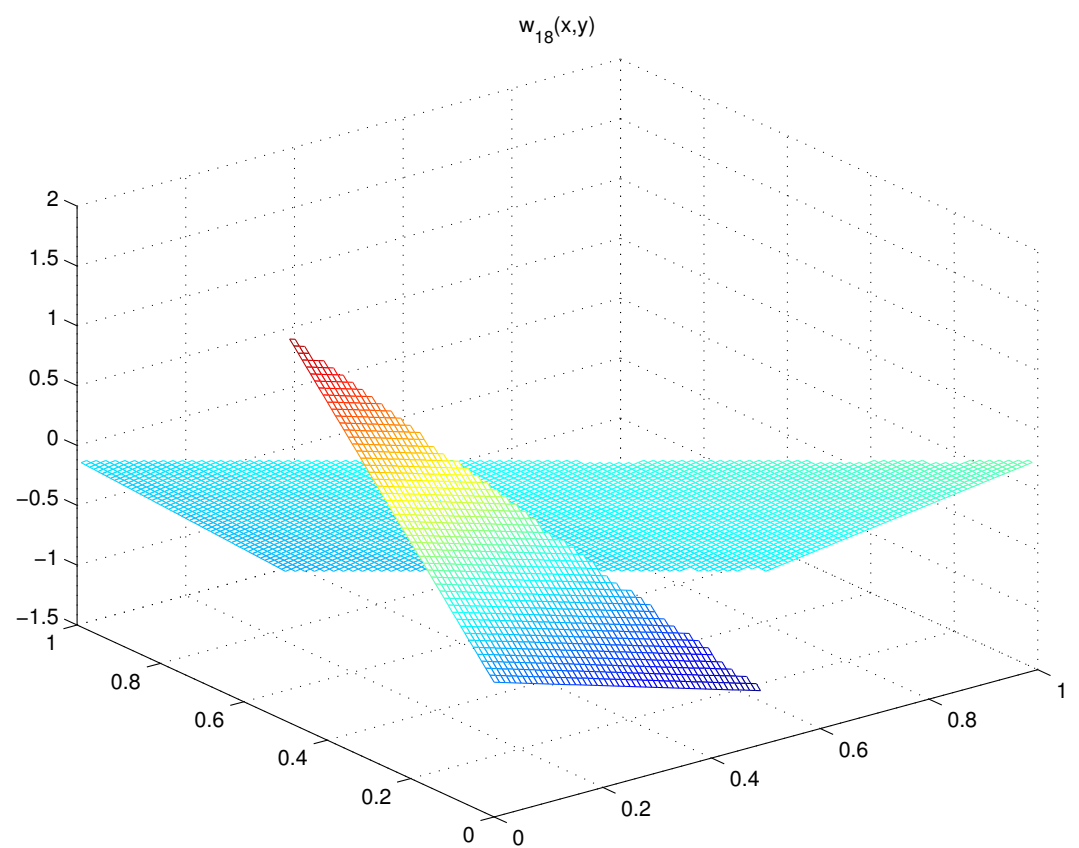

Figure 3.13: Wavelet function $w_{18}$ 


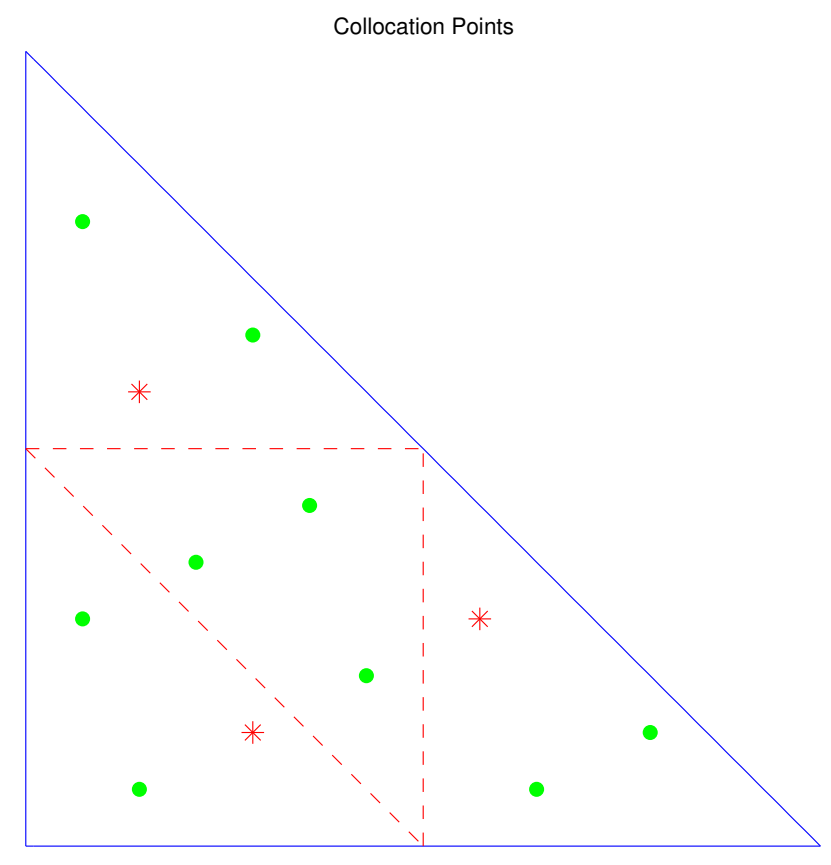

Figure 3.14: Collocation points of $L_{1}$

\subsection{Initial Collocation Functionals in $L_{1}$}

We define the set of initial collocation functionals at level 0 by

$$
L_{0}:=\left\{\ell_{0 j}=\delta_{t_{0 j}}, j \in \mathbb{Z}_{3}\right\}
$$

To find $L_{1}:=\left\{\ell_{1 j}: j \in \mathbb{Z}_{9}\right\}$, introduce a vector

$$
\mathbf{L}=\left[\ell_{10}, \ell_{11}, \ell_{12}, \ell_{13}, \ell_{14}, \ell_{15}, \ell_{16}, \ell_{17}, \ell_{18}\right]^{T},
$$

where, for $k \in \mathbb{Z}_{9}$,

$$
\ell_{1 k}=\sum_{m \in \mathbb{Z}_{12}} d_{k m} \delta_{t_{1 m}}, \quad t_{1 m}=\phi_{i} t_{0 j}, \quad m=3 i+j, \quad i \in \mathbb{Z}_{4}, j \in \mathbb{Z}_{3} .
$$

The 12 collocation points $t_{1 m}, m \in \mathbb{Z}_{12}$ are plotted in Fig. 3.14 (The three points marked by asterisk are the three initial collocation points $t_{0 j}, j \in \mathbb{Z}_{3}$ ). Let matrix $\mathbf{C}^{\prime} \in M_{9,12}$ with

$$
\mathbf{C}^{\prime}=\left[d_{k m}: k \in \mathbb{Z}_{9}, m \in \mathbb{Z}_{12}\right]
$$


Define

$$
\begin{aligned}
\delta_{\mathbf{t}}= & {\left[\delta_{t_{1,0}}, \delta_{t_{1,1}}, \delta_{t_{1,2}}, \delta_{t_{1,3}}, \delta_{t_{1,4}}, \delta_{t_{1,5}}, \delta_{t_{1,6}}, \delta_{t_{1,7}}, \delta_{t_{1,8}}, \delta_{t_{1,9}}, \delta_{t_{1,10}}, \delta_{t_{1,11}}\right]^{T} } \\
= & {\left[\delta_{\left(\frac{1}{14}, \frac{2}{7}\right)}, \delta_{\left(\frac{1}{7}, \frac{1}{14}\right)}, \delta_{\left(\frac{2}{7}, \frac{1}{7}\right)}, \delta_{\left(\frac{4}{7}, \frac{2}{7}\right)}, \delta_{\left(\frac{9}{14}, \frac{1}{14}\right)}, \delta_{\left(\frac{11}{14}, \frac{1}{7}\right)}, \delta_{\left(\frac{1}{14}, \frac{11}{14}\right)}, \delta_{\left(\frac{1}{7}, \frac{4}{7}\right)}, \delta_{\left(\frac{2}{7}, \frac{9}{14}\right)},\right.} \\
& \left.\delta_{\left(\frac{3}{7}, \frac{3}{14}\right)}, \delta_{\left(\frac{5}{14}, \frac{3}{7}\right)}, \delta_{\left(\frac{3}{14}, \frac{5}{14}\right)}\right]^{T} .
\end{aligned}
$$

Then we have

$$
\mathbf{L}=\mathbf{C}^{\prime} \delta_{\mathbf{t}}
$$

Again, to construct $\ell_{1 k}, k \in \mathbb{Z}_{9}$ having vanishing moment, require

$$
\begin{aligned}
& \left\langle\ell_{1 k}, 1\right\rangle=0 \\
& \left\langle\ell_{1 k}, x\right\rangle=0 \\
& \left\langle\ell_{1 k}, y\right\rangle=0 \\
& \left\langle\ell_{1 k}, w_{1 j}\right\rangle=\delta_{k j},
\end{aligned}
$$

where $k, j \in \mathbb{Z}_{9}$. (3.32) leads to nine $12 \times 12$ linear systems. After solving these nine $12 \times 12$ linear systems, we find that

$$
\mathbf{C}^{\prime}=\left[\begin{array}{cccccccccccc}
0 & 0 & -\frac{1}{2} & 0 & 0 & 0 & 0 & -\frac{1}{2} & 0 & 0 & 0 & 1 \\
0 & 0 & 0 & -\frac{1}{2} & 0 & 0 & 0 & -\frac{1}{2} & 0 & 0 & 1 & 0 \\
0 & 0 & -\frac{1}{2} & -\frac{1}{2} & 0 & 0 & 0 & 0 & 0 & 1 & 0 & 0 \\
0 & 0 & \frac{1}{2} & -\frac{1}{2} & 0 & 0 & 0 & -1 & 1 & 0 & 0 & 0 \\
0 & 0 & \frac{1}{2} & 0 & 0 & 0 & 1 & -\frac{3}{2} & 0 & 0 & 0 & 0 \\
0 & 0 & 0 & -\frac{3}{2} & 0 & 1 & 0 & \frac{1}{2} & 0 & 0 & 0 & 0 \\
0 & 0 & -\frac{1}{2} & -1 & 1 & 0 & 0 & \frac{1}{2} & 0 & 0 & 0 & 0 \\
0 & 1 & -\frac{3}{2} & \frac{1}{2} & 0 & 0 & 0 & 0 & 0 & 0 & 0 & 0 \\
1 & 0 & -1 & \frac{1}{2} & 0 & 0 & 0 & -\frac{1}{2} & 0 & 0 & 0 & 0
\end{array}\right] .
$$

To this point we have obtained desired $w_{1 j}$ and $\ell_{1 j}, j \in \mathbb{Z}_{9}$ having vanishing moment. To find the remaining multiscale wavelets $w_{i j}$ and functionals $\ell_{i j}$, for $i \geq 2, j \in w(i)$, simply apply (2.40) and (2.50). 


\subsection{Properties of the Wavelet Basis Functions and the Collocation Functionals}

We examine properties of the wavelet basis functions and the collocation functionals constructed above. These properties are crucial for developing fast wavelet collocation methods. Next several lemmas tells us these properties.

Lemma 3.1 The constructed basis function $w_{i j}, i \in \mathbb{N}_{0}, j \in \mathbb{Z}_{w(i)}$ are locally supported and their supports are shrinking as level $i$ increases.

Proof: for $i>1$, the support of $w_{i j}$ is contained in $S_{i j}=\phi_{\mathbf{e}}(E)=E_{i-1, \mu(\mathbf{e})}$, where $j=$ $9 \mu(\mathbf{e})+l, l \in \mathbb{Z}_{9}$. Note $\phi_{\mathbf{e}}$ is a contractive mapping by the contractivity of $\phi_{i}, i \in \mathbb{Z}_{4}$. Hence, $S_{i j}$ is shrinking as $i$ increases.

Lemma 3.2 For any $i, i^{\prime} \in \mathbb{N}_{0}$,

$$
\left\langle\ell_{i^{\prime} j^{\prime}}, w_{i j}\right\rangle=\delta_{i i^{\prime}} \delta_{j j^{\prime}}, \quad(i, j),\left(i^{\prime}, j^{\prime}\right) \in \mathbb{U}, i \leq i^{\prime}
$$

and

$$
\sum_{j \in \mathbb{Z}_{w(i)}}\left|\left\langle\ell_{i^{\prime} j^{\prime}}, w_{i j}\right\rangle\right| \leq \frac{81}{28}, \quad(i, j),\left(i^{\prime}, j^{\prime}\right) \in \mathbb{U}, i>i^{\prime}
$$

Further we have

$$
\gamma<\mu^{k / 2}-1
$$

where, $\gamma$ is defined in (3.37), and $k$ is the total degree of the pieced polynomial spaces $\mathbb{W}_{n}, n \in$ $\mathbb{N}_{0}$.

Proof: (3.34) is proved in Lemma 4.3. Let $\mathbf{C}_{\mathbf{1}}=\left[c_{i j}, i \in \mathbb{Z}_{9}, j \in \mathbb{Z}_{3}\right]$, where $c_{i j}$ are elements of $\mathbf{C}$ (see (3.22)). Note $\left\|\mathbf{C}_{\mathbf{1}}\right\|_{1}=\frac{27}{28}$, and $\left\|\mathbf{C}^{\prime}\right\|_{\infty}=3$. Set

$$
\gamma=\max \left\{\left\|\mathbf{C}_{\mathbf{1}}\right\|_{1},\left\|\mathbf{C}^{\prime}\right\|_{\infty}\left\|\mathbf{C}_{\mathbf{1}}\right\|_{1}\right\}=\frac{81}{28} .
$$

By Lemma 5.2 of [CMX3], which says $\sum_{j \in \mathbb{Z}_{w(i)}}\left|\left\langle\ell_{i^{\prime} j^{\prime}}, w_{i j}\right\rangle\right| \leq \gamma$, we have (3.35). Direct computation shows (3.36) with $k=2$. 
Yi Wang Chapter 3. Wavelets and collocation functionals on the Unit Triangle

Lemma 3.3 The constructed wavelets $w_{i j}$ and collocation functionals $\ell_{i j}$ for $i \in \mathbb{N}_{0}, j \in$ $\mathbb{Z}_{w(i)}$ have vanishing moment of degree 2.

Proof: From (3.32), we easily see

$$
\left\langle\ell_{i^{\prime} j^{\prime}}, w_{0 j}\right\rangle=\left\langle\ell_{1 l^{\prime}}, w_{0 j} \circ \phi_{\mathbf{e}^{\prime}}\right\rangle=0, \quad j \in \mathbb{Z}_{3}
$$

Now consider $\mathcal{T}_{\mathbf{e}}$ as an operator from $L^{2}(E)$ to $L^{2}(E)$ and let $\mathcal{T}_{\mathbf{e}}^{*}$ be the adjoint of $\mathcal{T}_{\mathbf{e}}$. It is easy to see that for $y \in L^{2}(E)$

$$
\mathcal{T}_{\mathbf{e}}^{*} y=J_{\phi_{\mathbf{e}}} y \circ \phi_{\mathbf{e}}
$$

where $J_{\phi_{\mathbf{e}}}$ is the Jacobi of mapping $\phi_{\mathbf{e}}$. Therefore,

$$
\left(w_{i j}, w_{0 j^{\prime}}\right)=\left(\mathcal{T}_{\mathbf{e}} w_{1 l}, w_{0 j^{\prime}}\right)=\left(w_{1 l}, \mathcal{T}_{\mathbf{e}}^{*} w_{0 j^{\prime}}\right)=0
$$

The last equality holds because $\mathcal{T}_{\mathbf{e}}^{*} w_{0 j^{\prime}}$ is a polynomial of total degree $\leq k-1=1$, with $k=2$ and $w_{1 l}$ satisfies (3.17).

Thus for any polynomials $p \in \pi_{2}$,

$$
\left\langle\ell_{i j}, p\right\rangle=0, \quad\left(w_{i j}, p\right)=0, \quad(i, j) \in \mathbb{U}, i \geq 1
$$

where $\pi_{k}$ is the space of polynomials total degree less than $k$.

Lemma 3.4 Both basis functions and collocation functionals are uniformly bounded.

Proof: For $(i, j) \in \mathbb{U}, i \geq 2, j=\mu(\mathbf{e}) r+l, l \in \mathbb{Z}_{r}$,

$$
\left|\left\langle\ell_{i j}, v\right\rangle\right|=\left|\left\langle\ell_{1 l}, v \circ \phi_{\mathbf{e}}\right\rangle\right| \leq\left\|\mathbf{C}^{\prime}\right\|_{\infty}\|v\|_{\infty}
$$

and

$$
\left\|w_{i j}\right\|_{\infty} \leq\left\|w_{1 l} \circ \phi_{\mathbf{e}}^{-1} \chi_{\phi_{\mathbf{e}}(E)}\right\|_{\infty} \leq\|\mathbf{C}\|_{\infty} \max _{j \in \mathbb{Z}_{12}}\left\|\psi_{j}\right\|_{\infty} .
$$

Lemma 3.5 The dimension of spaces $\mathbb{F}_{n}$ and $\mathbb{W}_{n}$ grows exponentially in $n$ and the diameters $d_{n}$ decay exponentially in $n$ too. where

$$
d_{n}:=\max \left\{d\left(S_{n m}\right): m \in \mathbb{Z}_{w(n)}\right\}, \quad n \in \mathbb{N}_{0} .
$$


Proof: We have $\operatorname{dim} \mathbb{W}_{0}=3, \operatorname{dim} \mathbb{F}_{n}=3.4^{n}, n \in \mathbb{N}_{0}$, and $\operatorname{dim} \mathbb{W}_{n}=9.4^{n-1}, n \geq 1, n \in \mathbb{Z}$. For the diameters we have $d_{0}=\sqrt{2}$ and $d_{n}=\sqrt{2} \frac{1}{2^{n-1}}=2^{3 / 2-n}$.

Lemma 3.6 There exists constant $\theta_{2}$ and $\theta_{3}$ such that for all $n \in \mathbb{N}_{0}$ and $v$ having form

$$
\begin{gathered}
v:=\sum_{(i, j) \in \mathbb{U}_{n}} v_{i j} w_{i j} \\
\theta_{2}\|\mathbf{v}\|_{\infty} \leq\|v\|_{\infty} \leq \theta_{3}(n+1)\left\|\mathbf{E}_{n} \mathbf{v}\right\|_{\infty}
\end{gathered}
$$

where $\mathbf{v}:=\left[v_{i j}:(i, j) \in \mathbb{U}_{n}\right]^{T}$.

Proof: The first inequality follows from Proposition 5.6 of [CMX3]. For the second inequality, we provide here a slight different proof. Define $\left\{\zeta_{i j}:(i, j) \in \mathbb{U}\right\}$ by:

$$
\zeta_{0 j}:=w_{0 j}, \quad j \in \mathbb{Z}_{3}
$$

For $j \in \mathbb{Z}_{9}$, find vector $\mathbf{c}_{j s}^{\prime \prime}:=\left[c_{j s}^{\prime \prime}: s \in \mathbb{Z}_{12}\right]$ such that

$$
\zeta_{1 j}:=\sum_{s \in \mathbb{Z}_{12}} c_{j s}^{\prime \prime} \psi_{s}
$$

satisfies

$$
\left\langle\ell_{0 j^{\prime}}, \zeta_{1 j}\right\rangle=0, \quad j^{\prime} \in \mathbb{Z}_{3}
$$

and

$$
\left\langle\ell_{1 j^{\prime}}, \zeta_{1 j}\right\rangle=\delta_{j j^{\prime}}, \quad j^{\prime} \in \mathbb{Z}_{9}
$$

Further define

$$
\zeta_{i j}:=\mathcal{T}_{\mathbf{e}} \zeta_{1 l}
$$

A direct computation shows that

$$
\left\langle\ell_{i^{\prime} j^{\prime}}, \zeta_{i j}\right\rangle=\delta_{i i^{\prime}} \delta_{j j^{\prime}}, \quad(i, j),\left(i^{\prime}, j^{\prime}\right) \in \mathbb{U} .
$$

[CMX3] proves in its Proposition 5.6 that there exists a constant $\theta_{3}$, for all $i \in \mathbb{N}_{0}$,

$$
\sup _{t \in \Omega} \sum_{j \in \mathbb{Z}_{w(i)}}\left|\zeta_{i j}(t)\right| \leq \theta_{3}
$$


For $(i, j) \in \mathbb{U}_{n},\left\{w_{i j}\right\}$ and $\left\{\zeta_{i j}\right\}$ are two different bases of $\mathbb{F}_{n}$. So we have

$$
v=\sum_{(i, j) \in \mathbb{U}_{n}} v_{i j} w_{i j}=\sum_{(i, j) \in \mathbb{U}_{n}} \tilde{v}_{i j} \zeta_{i j} .
$$

Observe that $\mathbf{E}_{n} \mathbf{v}=\tilde{\mathbf{v}}$, where $\tilde{\mathbf{v}}:=\left[\tilde{v}_{i j}:(i, j) \in \mathbb{U}_{n}\right]^{T}$. Now

$$
\|v\|_{\infty}=\left\|\sum_{(i, j) \in \mathbb{U}_{n}} \tilde{v}_{i j} \zeta_{i j}\right\|_{\infty} \leq\left\|\tilde{\mathbf{v}}_{\infty} \sum_{(i, j) \in \mathbb{U}_{n}} \zeta_{i j}\right\|_{\infty} \leq \tilde{\mathbf{v}}_{\infty}\left\|\sum_{i \in \mathbb{Z}_{n+1}} \sum_{j \in \mathbb{Z}_{w(i)}} \zeta_{i j}\right\|_{\infty} \leq \theta_{3}(n+1)\left\|\mathbf{E}_{n} \mathbf{v}\right\|_{\infty} .
$$

This completes the proof.

Lemma 3.7 Let $\mathcal{P}_{n}$ be the projection operator from $\mathbb{X}$ onto $\mathbb{F}_{n}$ defined by the requirement that

$$
\left\langle\ell_{i j}, \mathcal{P}_{n} x\right\rangle=\left\langle\ell_{i j}, x\right\rangle,(i, j) \in \mathbb{U}_{n}
$$

For $v \in L^{\infty}(E)$ we set

$$
\mathcal{P}_{n} v=\sum_{(i, j) \in \mathbb{U}_{n}} v_{i j} w_{i j}
$$

then

$$
\lim _{n \rightarrow \infty}\left\|\mathcal{P}_{n} x-x\right\|_{\infty}=0
$$

Proof: Since $\mathbb{F}_{n}$ is a space of piecewise polynomials, the operator $\mathcal{P}_{n}$ converges pointwise to the identity operator $\mathcal{I}$ in $L^{\infty}(E)$ as $n \rightarrow \infty$ by results from [AGS]. In other words, (3.53) holds.

Remark: For $x \in \mathbb{X}$, assume $\mathcal{P}_{n} x=\sum_{(i, j) \in \mathbb{U}_{n}} v_{i j} w_{i j}$. If we define a vector $\mathbf{L}_{n}:=\left\langle\ell_{i j}, x\right\rangle,(i, j) \in$ $\mathbb{U}_{n}$, then

$$
\mathbf{E}_{n} \mathbf{v}=\mathbf{L}_{n} \quad \text { or } \mathbf{v}=\mathbf{E}_{n}^{-1} \mathbf{L}_{n}
$$

Lemma 3.8 There exists a positive constant $c$ such that for $u \in W^{k, \infty}(E)$

$$
\operatorname{dist}\left(u, \mathbb{F}_{n}\right) \leq c \mu^{-k n / 2}\|u\|_{k, \infty}
$$

where for $\alpha:=\left[\alpha_{i} \in \mathbb{N}_{0}: i \in \mathbb{Z}_{2}\right],|\alpha|:=\sum_{i \in \mathbb{Z}_{2}} \alpha_{i}$,

$$
\|u\|_{k, \infty}:=\max \left\{\left\|D^{\alpha} u\right\|_{\infty}:|\alpha| \leq k\right\}
$$

and $\mu$ is the number of contractive mappings $\phi_{i}, i \in \mathbb{Z}_{\mu}$, as in (3.1), $\mu=4$. 
Proof: Since $\mathbb{F}_{n}$ are the spaces of piecewise polynomials of total degree $\leq k-1$ and our assumption that $E$ is a star-shaped set, then there exists a positive constant $c$ such that

$$
\operatorname{dist}\left(u, \pi_{k}\right) \leq c(d(E))^{k}\|u\|_{k, \infty}
$$

This together with (2.9) competes the proof.

\subsection{Reconstruction of Functions}

Let $E$ be the unit right triangle, $P_{0}=(0,0), P_{1}=(1,0)$. The vertices of the domain $\hat{E}:=\phi_{\mathbf{e}}(E)$ corresponding to $P_{0}, P_{1}$ are denoted by $\hat{P}_{i}, i \in \mathbb{Z}_{2}$, namely, $\hat{P}_{i}=\phi_{\mathbf{e}}\left(P_{i}\right), i \in \mathbb{Z}_{2}$. Further $\hat{P}_{i}=\left(\hat{x}_{i}, \hat{y}_{i}\right), i \in \mathbb{Z}_{2}$. Observe that

$$
\hat{E}:=\phi_{\mathbf{e}}(E)= \begin{cases}\left\{(s, t): \hat{x}_{0} \leq s \leq \hat{x}_{1}, \hat{y}_{0} \leq t \leq-s+\left(\hat{x}_{1}+\hat{y}_{1}\right)\right\} & \text { if } \hat{x}_{0} \leq \hat{x}_{1}, \\ \left\{(s, t): \hat{x}_{1} \leq s \leq \hat{x}_{0},-s+\left(\hat{x}_{1}+\hat{y}_{1}\right) \leq t \leq \hat{y}_{0}\right\} & \text { if } \hat{x}_{0} \geq \hat{x}_{1} .\end{cases}
$$

Let $u$ be the solution of $(2.2)$ in $\mathbb{X}, u_{n}$ be the approximation of $u$ sought by wavelet collocation scheme in $\mathbb{F}_{n}$. We have known from (2.16) that

$$
u_{n}(x):=\sum_{(i, j) \in \mathbb{U}_{n}} u_{i j} w_{i j}(x)
$$

where, $\mathbf{u}_{n}=\left[u_{i j}:(i, j) \in \mathbb{U}_{n}\right]$ is found by previously described wavelet collocation scheme.

Since each wavelet $w_{i j}$ has compact support, many terms in the summation of (3.58) are zeros. We need to determine if $w_{i j}(x)$ is vanished or not. If not, evaluate the value $w_{i j}(x)$. By (2.40), (2.39) and (2.34) we have

$$
w_{i j}(x)=\mathcal{T}_{\mathbf{e}} w_{1 l}(x)=w_{1 l}\left(\phi_{\mathbf{e}}^{-1}(x)\right) \chi_{\phi_{\mathbf{e}}(E)}(x),
$$

where $j=\mu(\mathbf{e}) r+l$, e $\in \mathbb{Z}_{\mu}^{i-1}, l \in \mathbb{Z}_{r}, r=9, \mu=4$.

We describe a fast algorithm in the following to compute $u_{n}(x)$. Assume $x$ is given and $\mathbf{u}_{n}=\left[u_{i j}:(i, j) \in \mathbb{U}_{n}\right]$ is found.

\section{Algorithm:}

1. Compute $u_{n}(x):=\sum_{(i, j) \in \mathbb{U}_{1}} u_{i j} w_{i j}(x)$; 
2. for $i=2 ; i \leq n$; step 1 , loop

$$
\begin{aligned}
& \text { for } j=0 ; j<\mu^{i-1} \text {; step } 1 \text {, loop } \\
& \quad \text { find } \mathbf{e} \in \mathbb{Z}_{\mu}^{i-1} \text {, such that } j=\mu(\mathbf{e}) ; \\
& \quad \text { if } x \in \phi_{\mathbf{e}}(E) \text {, compute the } r \text { evaluations } w_{1 l}\left(\phi_{\mathbf{e}}^{-1}(x)\right), l \in \mathbb{Z}_{r} \text {, and sum them up. }
\end{aligned}
$$$$
\text { Then jump out of the } j \text { loop, go to the next } i \text { loop; }
$$$$
\text { if } x \notin \phi_{\mathbf{e}}(E) \text {, continue next } j \text { loop; }
$$

end loop;

end loop;

Note in (3.58) for each level $i \geq 1$ and fixed $x \in E$, , only $r$ terms in the summation are nonzero, namely, only $r$ terms of $\chi_{\phi_{\mathbf{e}}(E)}(x)=1$. Also note to compute $w_{i j}(x)$, instead of directly using the expression of $w_{i j}$, we always go back to the first level $w_{1 l}$ by the recursive formula (3.59). 


\section{Chapter 4}

\section{Computation of Discrete Systems and Their Solutions}

We now turn our attention to compute matrices $\mathbf{E}_{n}$ and $\mathbf{K}_{n}$ of the discretized system (2.18). Their elements are expressed by (2.19) and (2.20) respectively. To compute these entries, we shall need to find $\ell_{i j}, w_{i j}$, for $i \geq 2, j \in \mathbb{Z}_{w(i)}$. Although (2.40) and (2.50) give expressions to find $w_{i j}, \ell_{i j}, i \geq 2, j \in \mathbb{Z}_{w(i)}$ respectively, they are not convenient for actual computing. More direct relations to wavelets and collocation functionals of level 1 are needed to easily compute $w_{i j}, \ell_{i j}$, for $i \geq 2, j \in \mathbb{Z}_{w(i)}$. Again we set $\mu=4$ and $r=9$. By (2.50) and (3.28) it is easy to see that

$$
\ell_{i j}=\sum_{m \in \mathbb{Z}_{12}} d_{l m} \delta_{t_{i m}}, \quad i \geq 2, j \in \mathbb{Z}_{w(i)}
$$

where, $l=j \bmod r ; d_{l m}, l \in \mathbb{Z}_{9}, m \in \mathbb{Z}_{12}$ are defined in (3.29), and

$$
t_{i m}=\phi_{\mathbf{e}}\left(t_{1 m}\right), \quad m \in \mathbb{Z}_{12}
$$

with $\mathbf{e} \in \mathbb{Z}_{\mu}^{i-1}$. For wavelets $w_{i j}, i \geq 2, j \in \mathbb{Z}_{w(i)}$, in view of (2.40) we have

$$
w_{i j}=w_{1 l} \circ \phi_{\mathbf{e}}^{-1}(t) \chi_{\phi_{\mathbf{e}}(E)}(t)
$$

where $j=\mu(\mathbf{e}) r+l$, e $\in \mathbb{Z}_{\mu}^{i-1}$, and $l \in \mathbb{Z}_{r}$. 
Yi Wang

\subsection{The Matrix $\mathrm{E}_{n}$}

Denote the element of $\mathbf{E}_{n}$ by $E_{i^{\prime} j^{\prime}, i j}$, where $(i, j),\left(i^{\prime}, j^{\prime}\right) \in \mathbb{U}_{n}$. By (2.20), (4.1) and (4.3), we obtain

$$
\begin{aligned}
E_{i^{\prime} j^{\prime}, i j} & =\left\langle\ell_{i^{\prime} j^{\prime}}, w_{i j}\right\rangle \\
& =\sum_{m \in \mathbb{Z}_{12}} d_{l^{\prime} m}\left\langle\delta_{t_{i^{\prime} m}}, w_{i j}\right\rangle \\
& =\sum_{m \in \mathbb{Z}_{12}} d_{l^{\prime} m}\left\langle\delta_{\phi_{\mathbf{e}^{\prime}}\left(t_{1 m}\right)}, w_{i j}\right\rangle \\
& =\sum_{m \in \mathbb{Z}_{12}} d_{l^{\prime} m} w_{1 l}\left(\phi_{\mathbf{e}}^{-1} \circ \phi_{\mathbf{e}^{\prime}}\left(t_{1 m}\right)\right) \chi_{\phi_{\mathbf{e}}(E)}\left(\phi_{\mathbf{e}^{\prime}}\left(t_{1 m}\right)\right) .
\end{aligned}
$$

where, $j=\mu(\mathbf{e}) r+l$, e $\in \mathbb{Z}_{\mu}^{i-1}, l \in \mathbb{Z}_{r}$ and $j^{\prime}=\mu\left(\mathbf{e}^{\prime}\right) r+l^{\prime}, \mathbf{e}^{\prime} \in \mathbb{Z}_{\mu}^{i^{\prime}-1}, l^{\prime} \in \mathbb{Z}_{r}$.

Define

$$
\mathbf{E}_{i^{\prime} i}:=\left[E_{i^{\prime} j^{\prime}, i j}: j^{\prime} \in \mathbb{Z}_{w\left(i^{\prime}\right)}, j \in \mathbb{Z}_{w(i)}\right]
$$

as the submatrix of $\mathbf{E}_{n}$ ( $\mathbf{K}_{i^{\prime} i}$ is similarly defined). We further partition $\mathbf{E}_{i^{\prime} i}$ ( also $\mathbf{K}_{i^{\prime} i}$ ) into blocks. Each block of $\mathbf{E}_{i^{\prime} i}$ corresponds to a pair of supports $\left(\hat{S}_{i^{\prime} j^{\prime}}, S_{i j}\right)$ which are supports of the pair $\left(\ell_{i^{\prime} j^{\prime}}, w_{i j}\right)$ respectively.

Definition 4.1 A set of elements in the submatrix $\mathbf{E}_{i^{\prime} i}$ (also in $\mathbf{K}_{i^{\prime} i}$ ) is called a block if they correspond to the same pair of supports $\left(\hat{S}_{i^{\prime} j^{\prime}}, S_{i j}\right)$ associated with the pair $\left(\ell_{i^{\prime} j^{\prime}}, w_{i j}\right)$.

Denote by the pair $\left(S\left(i^{\prime}\right), S(i)\right)$ the size of blocks in submatrix $\mathbf{E}_{i^{\prime} i}$ (also in $\mathbf{K}_{i^{\prime} i}$ ), with

$$
S(k)= \begin{cases}m, & k=0 \\ r, & \text { otherwise }\end{cases}
$$

where, $m=\operatorname{dim} \mathbb{F}_{0}$. In our case $m=3$. By Definition 4.1 , when $i^{\prime}=0$ and $i=0$, the block has size $m \times m$, and for $i^{\prime}, i>0$, each block has size $r \times r$. Therefore $\mathbf{E}_{i^{\prime} i}$ (also $\mathbf{K}_{i^{\prime} i}$ ) has $R\left(i^{\prime}\right)=\frac{w\left(i^{\prime}\right)}{S\left(i^{\prime}\right)}$ rows of blocks and $C(i)=\frac{w(i)}{S(i)}$ columns of blocks. For brevity, we write $\mathbf{E}_{i^{\prime} i}$ as

$$
\mathbf{E}_{i^{\prime} i}=\left[b_{j k}\right]_{j \in \mathbb{Z}_{R\left(i^{\prime}\right)}, k \in \mathbb{Z}_{C(i)}} .
$$

Following when describing the computation of matrix $\mathbf{E}_{i^{\prime} i}\left(\right.$ also $\left.\mathbf{K}_{i^{\prime} i}\right)$, we treat each $b_{j k}$ as a matrix element. 
Definition 4.2 For a matrix $\mathbf{A}=\left[a_{j k}\right]_{m \times n}$, where $m$ may not be equal to $n$ but one dividing the other. We call $\theta=\frac{\max \{m, n\}}{\min \{m, n\}}$ the shape index of $\mathbf{A}$. The main diagonal line of matrix $\mathbf{A}$ is defined as the set

$$
\left\{a_{j k}: j \theta \leq k<(j+1) \theta\right\} \quad \text { if } m \leq n,
$$

or

$$
\left\{a_{j k}: k \theta \leq j<(k+1) \theta\right\} \quad \text { if } m>n .
$$

For a given positive integer $c$ the $c$-th lower diagonal is defined as the set

$$
\left\{a_{j k}:(j-c) \theta \leq k<(j-c+1) \theta\right\} \quad \text { if } m \leq n
$$

or

$$
\left\{a_{j k}:(k+c) \theta \leq j<(k+c+1) \theta\right\} \quad \text { if } m>n
$$

The c-th upper diagonal is defined as the set

$$
\left\{a_{j k}:(j+c) \theta \leq k<(j+c+1) \theta\right\} \quad \text { if } m \leq n
$$

or

$$
\left\{a_{j k}:(k-c) \theta \leq j<(k-c+1) \theta\right\} \quad \text { if } m>n .
$$

Note, when $c=0$ we obtain the main diagonal in any case.

Next Lemma shows that $\mathbf{E}_{n}$ is upper triangular with diagonal blocks equal to identities of corresponding sizes. Moreover, the upper half of $\mathbf{E}_{n}$ is sparse already.

Lemma 4.3 For matrix $\mathbf{E}_{n}:=\left[E_{i^{\prime} j^{\prime}, i j}\right]$, where $i^{\prime}, i \in \mathbb{Z}_{n+1}, j \in \mathbb{Z}_{(i)}$, and $j^{\prime} \in \mathbb{Z}_{\left(i^{\prime}\right)}$.

1. $E_{i^{\prime} j^{\prime}, i j}=0$, for $i^{\prime}>i$;

2. $E_{i^{\prime} j^{\prime}, i j}=\delta_{i^{\prime} i} \delta_{j j^{\prime}}$, for $i^{\prime}=i$;

3. and, if $i^{\prime}<i$, in each submatrix $\mathbf{E}_{i^{\prime} i}$ with shape parameter $\theta_{i^{\prime} i}$, only elements on the main diagonal line are possibly nonzero. 
Proof: For $(i, j) \in \mathbb{U}_{n}$, with $i \geq 2$, there exists a unique pair of $\mathbf{e} \in \mathbb{Z}_{\mu}^{i-1}$ and $l \in \mathbb{Z}_{r}$ such that $j=\mu(\mathbf{e}) r+l$ and $w_{i j}=\mathcal{T}_{\mathbf{e}} w_{1 l}$. Likewise, for $\left(i^{\prime}, j^{\prime}\right) \in \mathbb{U}_{n}$, with $i^{\prime} \geq 2$, there exists a unique pair of $\mathbf{e}^{\prime} \in \mathbb{Z}_{\mu}^{i^{\prime}-1}$ and $l^{\prime} \in \mathbb{Z}_{r}$ such that $j^{\prime}=\mu\left(\mathbf{e}^{\prime}\right) r+l^{\prime}$ and $\ell_{i^{\prime} j^{\prime}}=\mathcal{L}_{\mathbf{e}^{\prime}} \ell_{1 l^{\prime}}$. When $i=i^{\prime}$, it follows from (2.49) and (2.45) that

$$
\left\langle\ell_{i^{\prime} j^{\prime}}, w_{i j}\right\rangle=\left\langle\mathcal{L}_{\mathbf{e}^{\prime}} \ell_{1 l^{\prime}}, \mathcal{T}_{\mathbf{e}} w_{1 l}\right\rangle=\left\langle\ell_{1 l^{\prime}}, w_{1 l}\right\rangle \delta_{\mathbf{e}^{\prime} \mathbf{e}}=\delta_{l^{\prime} l} \delta_{\mathbf{e e}^{\prime}}=\delta_{j^{\prime} j},
$$

since $\mathbf{e}=\mathbf{e}^{\prime}$. When $i<i^{\prime}$, let $\mathbf{e}_{1}^{\prime}=\left(e_{0}^{\prime}, \ldots, e_{i-2}^{\prime}\right), \mathbf{e}_{2}^{\prime}=\left(e_{i-1}^{\prime}, \ldots, e_{i^{\prime}-2}^{\prime}\right)$, then

$$
\left\langle\ell_{i^{\prime} j^{\prime}}, w_{i j}\right\rangle=\left\langle\mathcal{L}_{\mathbf{e}_{2}^{\prime}} \ell_{1 l^{\prime}}, w_{1 l}\right\rangle \delta_{\mathbf{e}_{1}^{\prime} \mathbf{e}}=\left\langle\ell_{1 l^{\prime}}, w_{1 l} \circ \phi_{\mathbf{e}_{2}^{\prime}}\right\rangle \delta_{\mathbf{e}_{1}^{\prime} \mathbf{e}} .
$$

Since $\phi_{\mathbf{e}_{2}^{\prime}}: E \rightarrow \phi_{\mathbf{e}_{2}^{\prime}}(E)$ is an affine mapping, $w_{1 l} \circ \phi_{\mathbf{e}_{2}^{\prime}}$ is a polynomial of total degree $\leq k-1$ in $\mathbb{F}_{0}$. By using (2.44), we have that

$$
\left\langle\ell_{i^{\prime} j^{\prime}}, w_{i j}\right\rangle=0, \quad(i, j),\left(i^{\prime}, j^{\prime}\right) \in \mathbb{U}_{n}, i<i^{\prime}
$$

when $i>i^{\prime}$, let $\mathbf{e}=\left(\mathbf{e}_{1}, \mathbf{e}_{2}\right)$, where $\mathbf{e}_{1}=\left(e_{0}, \ldots, e_{i^{\prime}-2}\right)$ and $\mathbf{e}_{2}=\left(e_{i^{\prime}-1}, \ldots, e_{i-2}\right)$, and

$$
\left\langle\ell_{i^{\prime} j^{\prime}}, w_{i j}\right\rangle=\left\langle\mathcal{L}_{\mathbf{e}^{\prime}} \ell_{1 l^{\prime}}, \mathcal{T}_{\mathbf{e}} w_{1 l}\right\rangle=\left\langle\ell_{1 l^{\prime}}, \mathcal{T}_{\mathbf{e}_{2}} w_{1 l}\right\rangle \delta_{\mathbf{e}^{\prime} \mathbf{e}_{1}}
$$

For the elements on the main diagonal line of submatrix $\mathbf{E}_{i^{\prime} i}, \mathbf{e}^{\prime}=\mathbf{e}_{1}$, and for those elements off the main diagonal line $\mathbf{e}^{\prime} \neq \mathbf{e}_{1}$. This completes the proof.

By (4.4) and (4.13), $\mathrm{t}$ for the elements on the main diagonal line of $\mathbf{E}_{i^{\prime} i}$,

$$
E_{i^{\prime} j^{\prime}, i j}=\sum_{m \in \mathbb{Z}_{12}} d_{l^{\prime} m} w_{1 l} \circ \phi_{\mathbf{e}_{2}}^{-1}\left(t_{1 m}\right) \chi_{\phi_{\mathbf{e}_{2}}(E)}\left(t_{1 m}\right) .
$$

(4.14) actually reduces the computation of the elements of $\mathbf{E}_{n}$ compared to (4.4). Fig. 4.1, Fig. 4.2 and Fig. 4.3 demonstrate computed full matrices $\mathbf{E}_{n}$ after discretizing the integral equation (6.1) with $n=2,3,4$ respectively. Clearly $\mathbf{E}_{n}$ is upper-triangular and sparse.

\subsection{The Matrix $\mathbf{K}_{n}$}

In view of (2.19), all the elements of $\mathbf{K}_{n}$ involve integrals of type $\int_{\Omega} K(x, y) w_{i j}(y) \mathrm{d} y$, where, $x \in \mathbb{R}^{2}$. Following theorem presents a perspective to compute this type of integrals. 


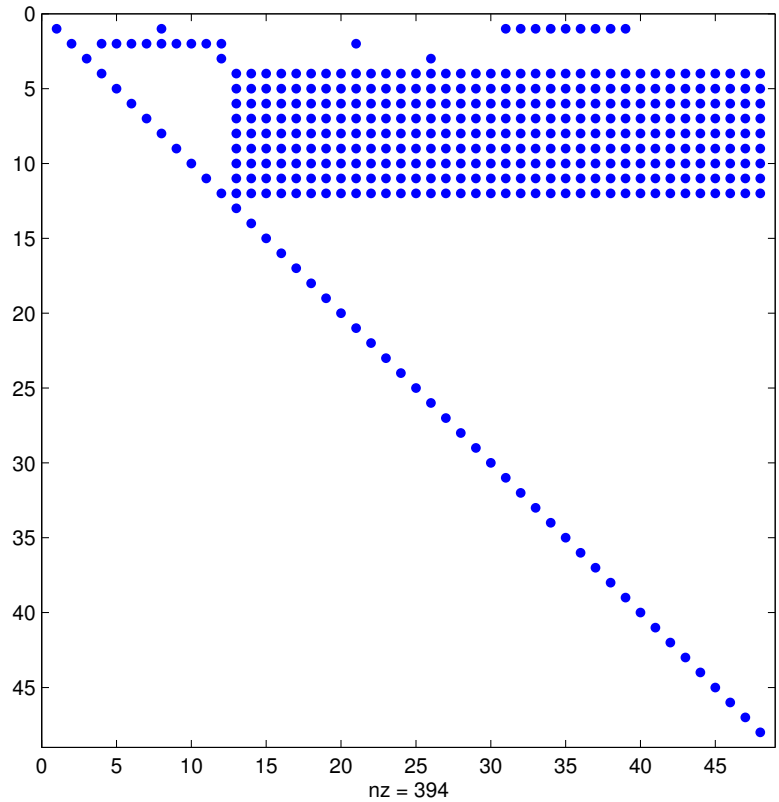

Figure 4.1: Matrix $\mathbf{E}_{2}$

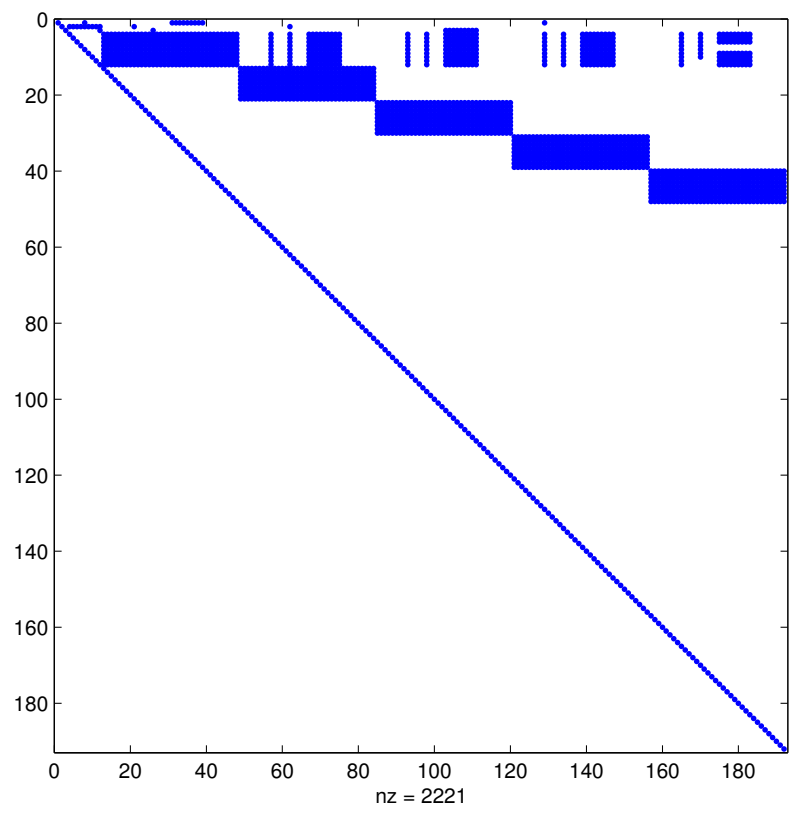

Figure 4.2: Matrix $\mathbf{E}_{3}$ 


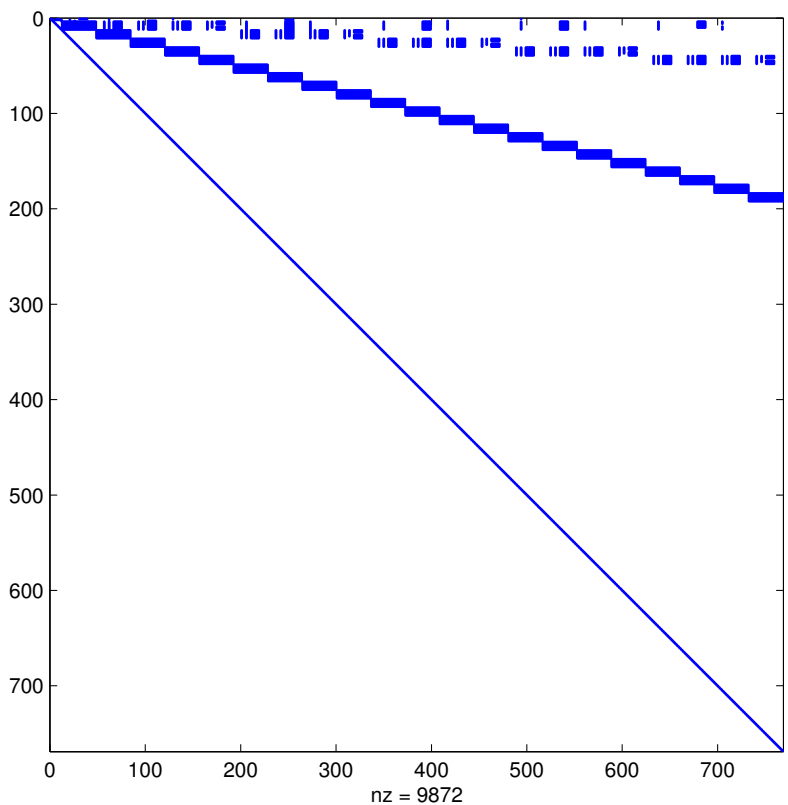

Figure 4.3: Matrix $\mathbf{E}_{4}$

\section{Theorem 4.4}

$$
\int_{\Omega} K(x, y) w_{i j}(y) d y=J_{\phi_{\mathbf{e}}} \int_{\Omega} K\left(x, \phi_{\mathbf{e}}(y)\right) w_{1 l}(y) \mathrm{d} y
$$

where $\mathbf{e} \in \mathbb{Z}_{\mu}^{i-1}, j=\mu(\mathbf{e})+l, l \in \mathbb{Z}_{r}, J_{\phi_{\mathbf{e}}}$ is the Jacobian of mapping $\phi_{\mathbf{e}}$. Namely,

$$
J_{\phi_{\mathbf{e}}}=J_{\phi_{e_{0}}} \cdot J_{\phi_{e_{1}}} \cdots J_{\phi_{e_{i-3}}} \cdot J_{\phi_{e_{i-2}}}
$$

and $w_{i j}$ is obtained by (2.40) for $i \geq 2, i \in \mathbb{Z}$.

Proof: We shall prove Theorem 4.4 by induction on $i$. Let $i=2, \mathbf{e} \in \mathbb{Z}_{\mu}^{1}$. Assume $\mathbf{e}=\left[e_{0}\right]$. Then

$$
\begin{aligned}
& \int_{\Omega} K(x, y) w_{2 j}(y) \mathrm{d} y \\
= & \int_{\Omega} K(x, y) \mathcal{T}_{\mathbf{e}} w_{1 l}(y) \mathrm{d} y \\
= & \int_{\Omega} K(x, y) \mathcal{T}_{e_{0}} w_{1 l}(y) \mathrm{d} y \\
= & \int_{\Omega} K(x, y) w_{1 l}\left(\phi_{e_{0}}^{-1}(y)\right) \mathrm{d} y \\
= & J_{\phi_{e_{0}}} \int_{\Omega} K\left(x, \phi_{e_{0}}(y)\right) w_{1 l}(y) \mathrm{d} y .
\end{aligned}
$$


The last equality is obtained by changing variable. Hence (4.15) holds for the case $i=2$. Assume (4.15) holds for $i<n$. for $i=n$, e $\in \mathbb{Z}_{\mu}^{n-1}$. Denote $\mathbf{e}=\left[e_{0} e_{1} \cdots e_{n-2}\right]$. We have

$$
\begin{aligned}
& \int_{\Omega} K(x, y) w_{n j}(y) \mathrm{d} y \\
= & \int_{\Omega} K(x, y) \mathcal{T}_{\mathbf{e}} w_{1 l}(y) \mathrm{d} y \\
= & \int_{\Omega} K(x, y) \mathcal{T}_{e_{0}, e_{1}, \ldots, e_{n-2}} w_{1 l}(y) \mathrm{d} y \\
= & \int_{\Omega} K(x, y) \mathcal{T}_{e_{0}} \mathcal{T}_{e_{1}, \ldots, e_{n-2}} w_{1 l}(y) \mathrm{d} y \\
= & J_{\phi_{e_{0}}} \int_{\Omega} K\left(x, \phi_{e_{0}}(y)\right) \mathcal{T}_{e_{1}, \ldots, e_{n-2}} w_{1 l}(y) \mathrm{d} y \\
= & J_{\phi_{\mathbf{e}}} \int_{\Omega} K\left(x, \phi_{\mathbf{e}}(y)\right) w_{1 l}(y) \mathrm{d} y .
\end{aligned}
$$

The last equality obtained by the induction assumption.

Theorem 4.4 facilitates us a convenient way to compute the integral on the left side of (4.15). Note $w_{i j}$ has compact support $\phi_{\mathbf{e}}(E)$. But by Theorem 4.4 we don't need to find the explicit expression for $w_{i j}$ and its support $\phi_{\mathbf{e}}(E)$.

We now look at how to compute elements of $\mathbf{K}_{n}$. Denote elements of $\mathbf{K}_{n}$ by $K_{i^{\prime} j^{\prime}, i j}$, where, $(i, j),\left(i^{\prime}, j^{\prime}\right) \in \mathbb{U}_{n}$. For $i, i \geq 2$, let $j=\mu(\mathbf{e}) r+l$, and $j^{\prime}=\mu\left(\mathbf{e}^{\prime}\right) r+l^{\prime}$, where, e $\in \mathbb{Z}_{\mu}^{i-1}$, $\mathbf{e}^{\prime} \in \mathbb{Z}_{\mu}^{i^{\prime}-1}$, and $l, l^{\prime} \in \mathbb{Z}_{r}$. By (2.19), (4.1), (4.2) and Theorem 4.4

$$
\begin{aligned}
K_{i^{\prime} j^{\prime}, i j} & =\left\langle\ell_{i^{\prime} j^{\prime}}, \mathcal{K} w_{i j}\right\rangle \\
& =\int_{\Omega}\left\langle\ell_{i^{\prime} j^{\prime}}, K(\cdot, y)\right\rangle w_{i j}(y) \mathrm{d} y \\
& =\sum_{m \in \mathbb{Z}_{12}} d_{l^{\prime} m} \int_{\Omega} K\left(\phi_{\mathbf{e}^{\prime}}\left(t_{1 m}\right), y\right) w_{i j}(y) \mathrm{d} y \\
& =\sum_{m \in \mathbb{Z}_{12}} d_{l^{\prime} m}\left|J_{\phi_{\mathbf{e}}}\right| \int_{\Omega} K\left(\phi_{\mathbf{e}^{\prime}}\left(t_{1 m}\right), \phi_{\mathbf{e}}(y)\right) w_{1 l}(y) \mathrm{d} y
\end{aligned}
$$

For cases of $i \leq 1$, or $i^{\prime} \leq 1$ or both, computation of $K_{i^{\prime} j^{\prime}, i j}$ is easier, and one can slightly modify (4.17) to obtain the desired formulae. Note, if $K(x, y)$ is a weakly singular kernel with discontinuity at $x=y$, in view of the integral in (4.17) the singular points of its integrand are at

$$
y_{s}=\phi_{\mathbf{e}}^{-1} \phi_{\mathbf{e}^{\prime}}\left(t_{1 m}\right), \quad m \in \mathbb{Z}_{12} .
$$

We point out that the singular point $y$ is not necessary in the domain $\Omega$. Following lemma addresses this issue. 
Lemma 4.5 For those elements $K_{i^{\prime} j^{\prime}, i j}$ on the main diagonal line (as defined in Definition 4.2) of the submatrix $\mathbf{K}_{i^{\prime} i}$, with $i, i^{\prime} \geq 2$, let $K(x, y)$ be a weakly singular kernel with singularity at $y=x$,

1. when $i^{\prime}=i$, the singular points are at $y_{s}=t_{1 m}$, where $m \in \mathbb{Z}_{12}$;

2. when $i>i^{\prime}$ the singular points are at $y_{s}=\phi_{\mathbf{e}_{2}}^{-1}\left(t_{1 m}\right)$, where $\mathbf{e}_{2} \in \mathbb{Z}_{i-i^{\prime}}$. Note, in this case $y$ is not necessarily in $\Omega$;

3. when $i<i^{\prime}$ the singular points are at $y_{s}=\phi_{\mathbf{e}_{2}^{\prime}}\left(t_{1 m}\right)$, where $\mathbf{e}_{2}^{\prime} \in \mathbb{Z}_{i^{\prime}-i}$. In this case $y$ is always in $\Omega$.

For all other elements off the main diagonal line in each block $\mathbf{K}_{i^{\prime} i}$, the integrals in (4.17) has no singular points.

Proof: We first prove the statements regarding elements on the main diagonal lines in each submatrix $\mathbf{K}_{i^{\prime} i}$ with $i^{\prime}, i \geq 2$. Again we assume $j=\mu(\mathbf{e}) r+l$, and $j^{\prime}=\mu\left(\mathbf{e}^{\prime}\right) r+l^{\prime}$, where, $\mathbf{e} \in \mathbb{Z}_{\mu}^{i-1}, \mathbf{e}^{\prime} \in \mathbb{Z}_{\mu}^{i^{\prime}-1}$, and $l, l^{\prime} \in \mathbb{Z}_{r}$

When $i^{\prime}=i$, e $=\mathbf{e}^{\prime}$, so $y_{s}=t_{1 m}$ by (4.18).

When $i>i^{\prime}$, let $\mathbf{e}=\left(\mathbf{e}_{1}, \mathbf{e}_{2}\right)$, where $\mathbf{e}_{1}=\left(e_{0}, \ldots, e_{i^{\prime}-2}\right)$ and $\mathbf{e}_{2}=\left(e_{i^{\prime}-1}, \ldots, e_{i-2}\right)$. For the elements on the main diagonal of $\mathbf{K}_{i^{\prime} i}, \mathbf{e}^{\prime}=\mathbf{e}_{1}$. Hence,

$$
y_{s}=\left(\phi_{\mathbf{e}_{1}} \phi_{\mathbf{e}_{2}}\right)^{-1} \phi_{\mathbf{e}^{\prime}}\left(t_{1 m}\right)=\phi_{\mathbf{e}_{2}}^{-1} \phi_{\mathbf{e}_{1}}^{-1} \phi_{\mathbf{e}^{\prime}}\left(t_{1 m}\right)=\phi_{\mathbf{e}_{2}}^{-1}\left(t_{1 m}\right)
$$

As $i>i^{\prime}$, the length of $\mathbf{e}_{2} \geq 1$. Since $\phi_{\mathbf{e}_{2}}$ is a contraction, so in this case $y_{s}$ may be outside of $\Omega$.

When $i<i^{\prime}$, let $\mathbf{e}^{\prime}=\left(\mathbf{e}_{1}^{\prime}, \mathbf{e}_{2}^{\prime}\right)$, where, $\mathbf{e}_{1}^{\prime}=\left(e_{0}^{\prime}, \ldots, e_{i-2}^{\prime}\right)$, and $\mathbf{e}_{2}^{\prime}=\left(e_{i-1}^{\prime}, \ldots, e_{i^{\prime}-2}^{\prime}\right)$. Then,

$$
y_{s}=\phi_{\mathbf{e}}^{-1} \phi_{\mathbf{e}_{1}^{\prime}} \phi_{\mathbf{e}_{2}^{\prime}}\left(t_{1 m}\right)=\phi_{\mathbf{e}_{2}^{\prime}}\left(t_{1 m}\right) .
$$

since $\mathbf{e}=\mathbf{e}_{1}^{\prime}$. Note in this case $y_{s}$ is always inside $\Omega$ since $\phi_{\mathbf{e}_{2}^{\prime}}$ is a contractive mapping.

For elements which are off the main diagonal lines of $\mathbf{K}_{i^{\prime} i}, \hat{S}_{i^{\prime} j^{\prime}}$ and $S_{i j}$ which correspond to the supports of the pair $\left(\ell_{i^{\prime} j^{\prime}}, w_{i j}\right)$ are disjoint except possible common boundary points. Recall that all the collocation points are interior points of $\hat{S}_{i^{\prime} j^{\prime}}$, therefore there are no singular points inside the domain of integration for integrals associated with those elements. 
Although we can compute elements of $\mathbf{K}_{n}$ by (4.17) and Theorem 4.4, when singular points are outside $\Omega$, it's not easy for us to pick up appropriate meshes to compute quadratures. Especially when singular points are very close to $\Omega$ but remaining outside of $\Omega$. In this case if we choose equally spaced meshes to subdivide domains of integration, it is obvious that it would be improper. If we intend to choose unequally spaced meshes, the question of how to uniformly deal with all four cases stated in Lemma 4.5 occurs. Next Theorem provides ways to uniformly treat all different types of integrals associated with elements of $\mathbf{K}_{n}$ with uniform accuracy.

Theorem 4.6 Let $P_{k}, k \in \mathbb{Z}_{3}$ be vertices of the original triangular domain $\Omega$ supported $w_{1 l}, l \in \mathbb{Z}_{r}$, where $P_{0}$ is the vertex of the right angle, and $P_{1}, P_{2}$ are indexed counterclockwisely (see Figure 4.4). Denote new vertices of the transformed domain $\phi_{\mathbf{e}}(\Omega)$ by $\hat{P}_{k}$, and $\hat{P}_{k}=$ $\phi_{\mathbf{e}}\left(P_{k}\right), k \in \mathbb{Z}_{3}$, where $\mathbf{e} \in \mathbb{Z}_{\mu}^{i-1}$ for $i \geq 2$. Denote coordinates of $\hat{P}_{k}$ by $\left(\hat{x}_{k}, \hat{y}_{k}\right)$. For $i, i^{\prime} \geq 2$, the elements $K_{i^{\prime} j^{\prime}, i j}$ of $\mathbf{K}_{n}$ can be computed by the following way:

$$
K_{i^{\prime} j^{\prime}, i j}=\sum_{m \in \mathbb{Z}_{12}} d_{l^{\prime} m} \int_{\hat{x}_{0}}^{\hat{x}_{1}} \int_{\hat{y}_{0}}^{-x+\left(\hat{x}_{1}+\hat{y}_{1}\right)} K\left(\phi_{\mathbf{e}^{\prime}}\left(t_{1 m}\right), \eta\right) w_{1 l}\left(\phi_{\mathbf{e}}^{-1}(\eta)\right) \mathrm{d} y \mathrm{~d} x
$$

where, $t_{1 m}, \eta, P_{k}, \hat{P}_{k} \in \mathbb{R}^{2}, k \in \mathbb{Z}_{3}$, and $\eta=(x, y)$.

Proof: Since $\phi_{\mathbf{e}}$ is a contractive affine mapping and also conformal, $\phi_{\mathbf{e}}(\Omega)$ is a right triangle too. The orientation of $\phi_{\mathbf{e}}(\Omega)$ is either as the lower left one in Fig. 4.4 or as the lower right one in Fig. 4.4.

Case 1: The new domain $\phi_{\mathbf{e}}(\Omega)$ is as the lower left one in Fig. 4.4. Note the hypotenuse $\hat{P}_{1} \hat{P}_{2}$ always has slope -1 and it's equation is

$$
y=-x+\left(\hat{x}_{1}+\hat{y}_{1}\right)
$$

Hence

$$
\begin{aligned}
K_{i^{\prime} j^{\prime}, i j} & =\sum_{m \in \mathbb{Z}_{12}} d_{l^{\prime} m} \int_{\phi_{\mathbf{e}}(\Omega)} K\left(\phi_{\mathbf{e}^{\prime}}\left(t_{1 m}\right), \eta\right) w_{i j}(\eta) \mathrm{d} \eta \\
& =\sum_{m \in \mathbb{Z}_{12}} d_{l^{\prime} m} \int_{\phi_{\mathbf{e}}(\Omega)} K\left(\phi_{\mathbf{e}^{\prime}}\left(t_{1 m}\right), \eta\right) w_{1 l}\left(\phi_{\mathbf{e}}^{-1}(\eta)\right) \mathrm{d} \eta \\
& =\sum_{m \in \mathbb{Z}_{12}} d_{l^{\prime} m} \int_{\hat{x}_{0}}^{\hat{x}_{1}} \int_{\hat{y}_{0}}^{-x+\left(\hat{x}_{1}+\hat{y}_{1}\right)} K\left(\phi_{\mathbf{e}^{\prime}}\left(t_{1 m}\right), \eta\right) w_{1 l}\left(\phi_{\mathbf{e}}^{-1}(\eta)\right) \mathrm{d} y \mathrm{~d} x
\end{aligned}
$$


The original domain $\Omega$

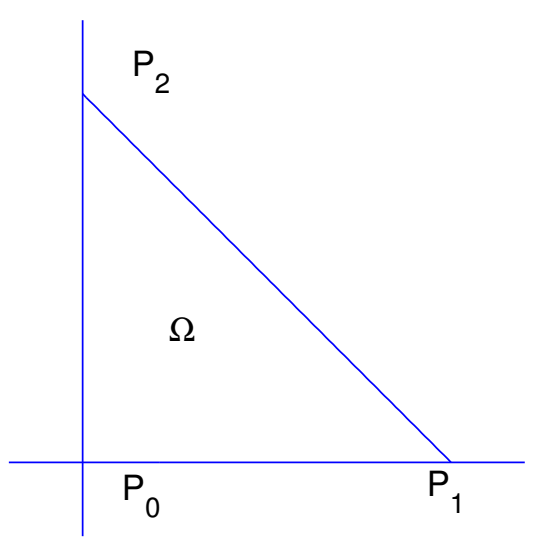

The new domain $\phi \mathrm{e}^{\Omega \text {, case } 1}$ The new domain $\phi \mathrm{e}^{\Omega \text {, case } 2}$
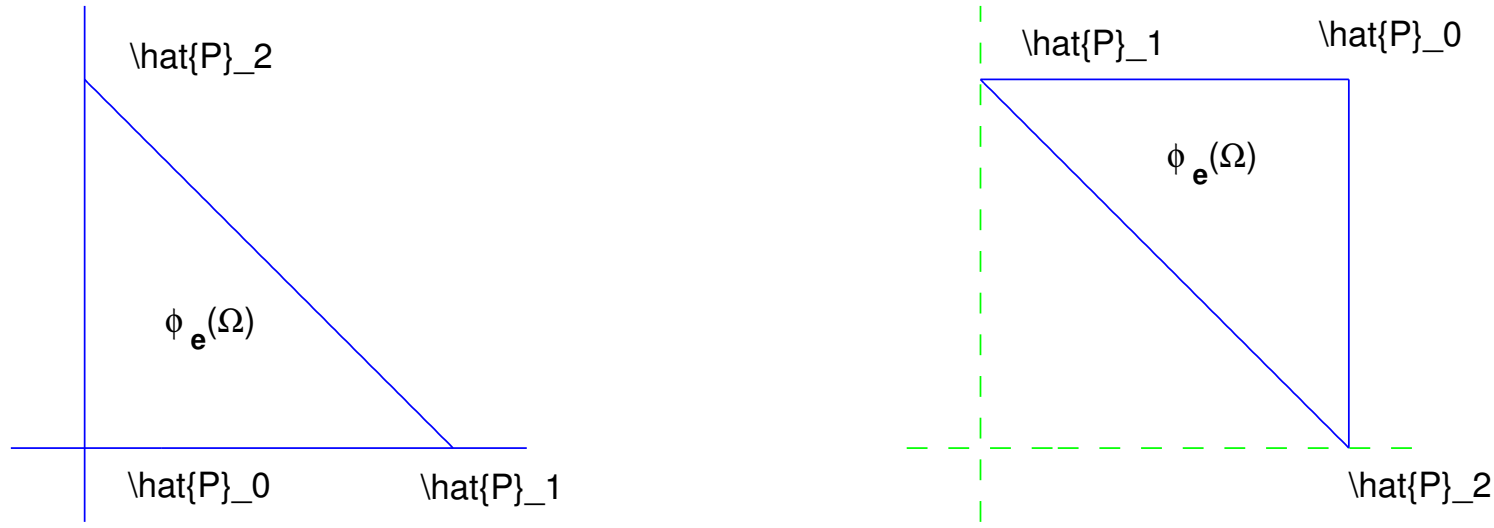

Figure 4.4: Domains of integration 
Case 2: The new domain $\phi_{\mathbf{e}}(\Omega)$ is as the lower right one in Fig. 4.4.

$$
\begin{aligned}
K_{i^{\prime} j^{\prime}, i j} & =\sum_{m \in \mathbb{Z}_{12}} d_{l^{\prime} m} \int_{\phi_{\mathbf{e}}(\Omega)} K\left(\phi_{\mathbf{e}^{\prime}}\left(t_{1 m}\right), \eta\right) w_{i j}(\eta) \mathrm{d} \eta \\
& =\sum_{m \in \mathbb{Z}_{12}} d_{l^{\prime} m} \int_{\phi_{\mathbf{e}}(\Omega)} K\left(\phi_{\mathbf{e}^{\prime}}\left(t_{1 m}\right), \eta\right) w_{1 l}\left(\phi_{\mathbf{e}}^{-1}(\eta)\right) \mathrm{d} \eta \\
& =\sum_{m \in \mathbb{Z}_{12}} d_{l^{\prime} m} \int_{\hat{x}_{1}}^{\hat{x}_{0}} \int_{-x+\left(\hat{x}_{1}+\hat{y}_{1}\right)}^{\hat{y}_{0}} K\left(\phi_{\mathbf{e}^{\prime}}\left(t_{1 m}\right), \eta\right) w_{1 l}\left(\phi_{\mathbf{e}}^{-1}(\eta)\right) \mathrm{d} y \mathrm{~d} x \\
& =\sum_{m \in \mathbb{Z}_{12}} d_{l^{\prime} m} \int_{\hat{x}_{0}}^{\hat{x}_{1}} \int_{\hat{y}_{0}}^{-x+\left(\hat{x}_{1}+\hat{y}_{1}\right)} K\left(\phi_{\mathbf{e}^{\prime}}\left(t_{1 m}\right), \eta\right) w_{1 l}\left(\phi_{\mathbf{e}}^{-1}(\eta)\right) \mathrm{d} y \mathrm{~d} x .
\end{aligned}
$$

This completes the proof.

Remark: when $i \leq 1$ or $i^{\prime} \leq 1$ or both, it is easier to compute $K_{i^{\prime} j^{\prime}, i j}$. Slightly modify (4.20) if necessary.

Since the point $\phi_{\mathbf{e}^{\prime}}\left(t_{1 m}\right), m \in \mathbb{Z}_{12}$ is always contained in $\Omega$, (4.20) allows an easy way to get an unequally spaced mesh on $\Omega$ with $\phi_{\mathbf{e}^{\prime}}\left(t_{1 m}\right)$ as the singular point. This will be detailly discussed in Section 4.3. Theorem 4.6 will be the actual method we adopt to compute elements of $\mathbf{K}_{n}$. Fig. 4.5 depicts the computed full matrix $\mathbf{K}_{3}$ from example in solving (6.1). For viewing effect, absolute values of matrix entries are drawn. After the wavelet transform, the matrix $\mathbf{K}_{n}$ is amazingly numerically sparse. Namely many entries are relatively very small, and the matrix appears as "finger" shape.

\subsection{An Adaptive Quadrature Rule}

All the elements of $\mathbf{K}_{n}$ need to compute

$$
\int_{a}^{b} \int_{c}^{d-y_{1}} K(x, y) w_{i j}(y) d y_{2} d y_{1}, \quad 0 \leq a, b, c, d \leq 1,
$$

where, $x, y \in \mathbb{R}^{2}$ with $x=\left(x_{1}, x_{2}\right), y=\left(y_{1}, y_{2}\right) . w_{i j}, i \in \mathbb{N}_{0}, j \in \mathbb{Z}_{w(i)}$ is a wavelet function. $K(x, y)$ is a weakly singular function on $\Omega \times \Omega$. Namely, for $x, y \in \Omega, x \neq y$, the kernel $K$ has continuous partial derivatives $D_{x}^{\alpha} D_{y}^{\beta} K(x, y)$ for $|\alpha| \leq k,|\beta| \leq k$. Moreover, there exists positive constants $\sigma$ and $\theta_{1}$ with $\sigma<2$ such that for $|\alpha|=|\beta|=k$ there holds

$$
\left|D_{x}^{\alpha} D_{y}^{\beta} K(x, y)\right| \leq \frac{\theta_{1}}{|x-y|^{\sigma+|\alpha|+|\beta|}} .
$$

While $K(x, y)$ is singular when $x=y$. Note $w_{i j}$ for $i \geq 1, j \in \mathbb{Z}_{w(i)}$ is a piecewise polynomial and has shrinking supports as $i$ increases. In general, there are two different cases: 


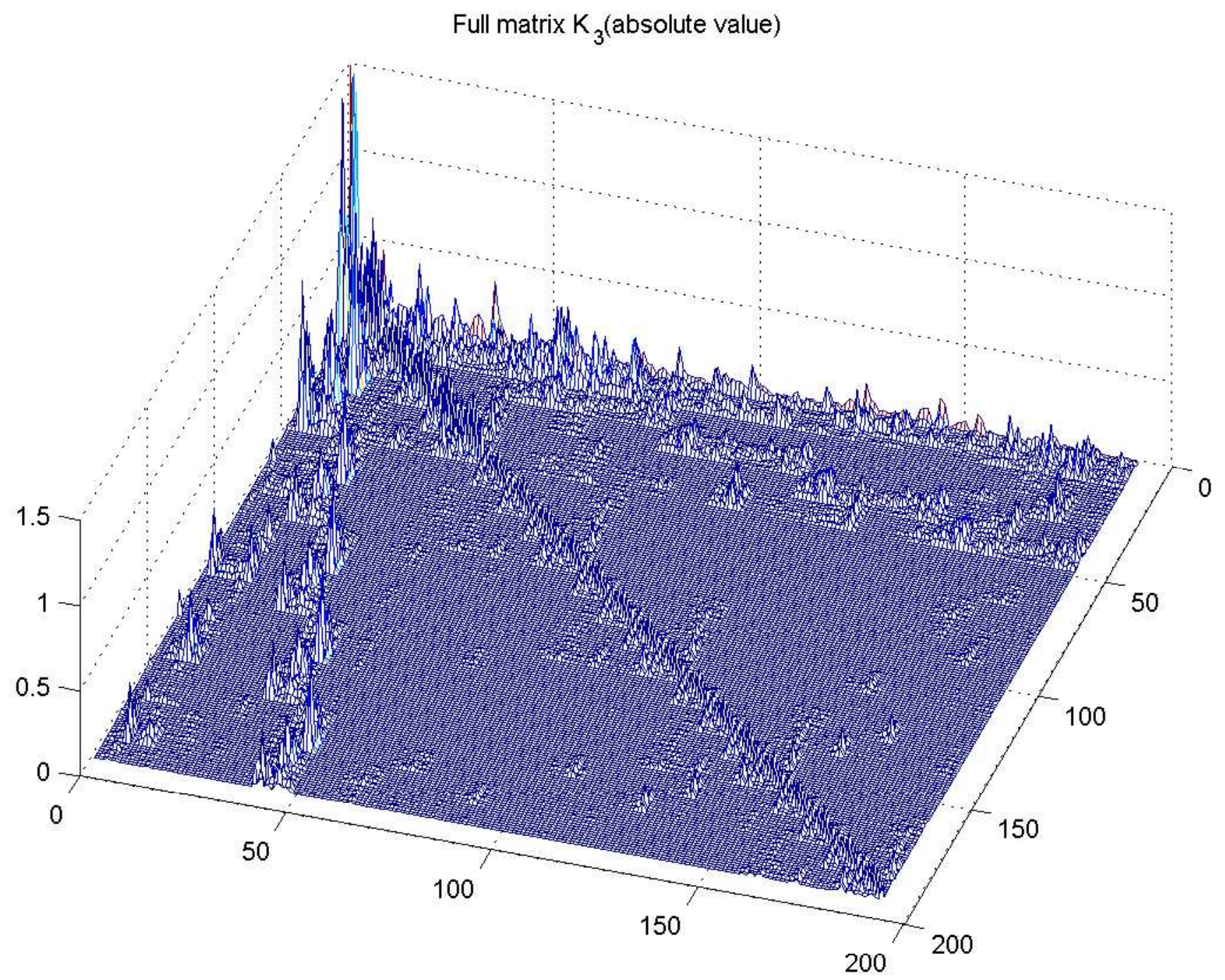

Figure 4.5: Full Matrix $\mathbf{K}_{3}$ 
1. the singular point $x$ is within the support of $w_{i j}$,

2. $x$ is not in the support of $w_{i j}$.

We want to have a uniform scheme to deal with these two cases and the shrinking supports of $w_{i j}$, nevertheless, uniform accuracies are desired for all cases. [KX] provides a good quadrature method for 1-D integrals with weakly singular kernels. we adapt the idea proposed therein to develop an efficient adaptive algorithm to compute double integrals equipped with weakly singular kernels. Rewrite (4.22) into

$$
I:=\int_{a}^{b} h\left(x ; y_{1}\right) \mathrm{d} y_{1},
$$

where, $h\left(x ; y_{1}\right):=\int_{c}^{d-y_{1}} K(x, y) w_{i j}(y) \mathrm{d} y_{2}$. Denote $\max \left\{x_{1}, 1-x_{1}\right\}$ by $c_{\max }$ and $\min \left\{x_{1}, 1-\right.$ $\left.x_{1}\right\}$ by $c_{\min }$. Note $1 \geq c_{\max } \geq \frac{1}{2}$ and $0 \leq c_{\min } \leq \frac{1}{2}$. We first obtain an uneven partition of $[a, b]$ by the following Algorithm:

1. Subdivide $\left[0, c_{\max }\right]$ into $n$ subintervals by the following way:

$$
\hat{t}_{j}=c_{\max }\left(\frac{j}{n}\right)^{q}, \quad j \in \mathbb{Z}_{n+1},
$$

where $n$ is determined by

$$
n=\left\lfloor m \frac{c_{\max }}{1 / 2}=2 m c_{\max }\right\rfloor .
$$

Note $\hat{t}_{0}=0$ and $\hat{t}_{n}=c_{\max } . m$ is a prescribed integer. $q=\frac{2 k+1}{1-\alpha}$ (see $[\mathrm{KX}]$ for detail). $k$ is the total degree of the interpolatory piecewise polynomial used in the compound quadrature. For a function $g, \alpha \in(0,1)$ is called the index of singularity. It's defined by

$$
\left|g^{(\beta)}(x)\right| \leq C|x|^{(-\alpha-\beta)}, \text { for } x \neq 0
$$

This notation was first introduced by Rice in [Ri].

2. Determine the number of subintervals $J$ in the shorter interval $\left[0, c_{\min }\right]$. Require that

$$
c_{\max } \cdot\left(\frac{J}{n}\right)^{q}=c_{\min }
$$

By (4.27), we can take

$$
J=\left\lceil\left(\frac{c_{\min }}{c_{\max }}\right)^{\frac{1}{q}} n\right\rceil .
$$


3. if $x_{1} \leq 1-x_{1}$

Set $t_{0}^{\prime}=0$. Let

$$
t_{J-j}^{\prime}=x_{1}-\hat{t}_{j}, \quad j \in \mathbb{Z}_{J},
$$

and

$$
t_{J+i}^{\prime}=x_{1}+\hat{t}_{i}, \quad i \in \mathbb{Z}_{n+1} .
$$

Note $t_{J}^{\prime}=x_{1}$, and $t_{J+n}^{\prime}=1$.

4. if $x_{1}>1-x_{1}$

$$
t_{n-j}^{\prime}=x_{1}-\hat{t}_{j}, \quad j \in \mathbb{Z}_{n+1},
$$

and

$$
t_{n+i}^{\prime}=x_{1}+\hat{t}_{i}, \quad i \in \mathbb{Z}_{J} .
$$

Note $t_{0}^{\prime}=0, t_{n}^{\prime}=x_{1}$ and we set $t_{n+J}^{\prime}=1$.

Define that $\triangle_{t^{\prime}}^{y_{1}}:=\left\{t_{i}^{\prime}: i \in \mathbb{Z}_{n+J+1}\right\}$. Let $\triangle\left(w_{i j}\right)$ be the set of discontinuous points of $w_{i j}$ along $y_{1}$-direction. Define

$$
\triangle_{a}^{b}:=\triangle_{t^{\prime}}^{y_{1}} \cup \triangle\left(w_{i j}\right) \cup\{a, b\} \cap[a, b]
$$

Reorder the points in $\triangle_{a}^{b}$ by an increasing order and rename them as

$$
a=t_{0}<t_{1}<\cdots<t_{m_{1}}=b .
$$

This is an desired partition of $[a, b]$. Now (4.24) becomes into

$$
I=\sum_{i=0}^{m_{1}-1} \int_{t_{i}}^{t_{i+1}} h(x ; \xi) \mathrm{d} \xi=\int_{-1}^{1} H(s) \mathrm{d} s
$$

where,

$$
H(s)=\sum_{i=0}^{m_{1}-1} \bar{t}_{i}^{-} h\left(x ; \bar{t}_{i}^{-} s+\bar{t}_{i}^{+}\right),
$$

with

$$
\bar{t}_{i}^{+}=\frac{t_{i+1}+t_{i}}{2}, \quad \bar{t}_{i}^{-}=\frac{t_{i+1}-t_{i}}{2} .
$$


Using Gaussian quadrature method with the interpolating polynomial of degree $k^{\prime}$, we obtain the quadrature value of $I$

$$
I_{m}=\sum_{j \in \mathbb{Z}_{k^{\prime}}} w_{j} H\left(u_{j}\right)
$$

where, $\left\{u_{i}\right\}, i \in \mathbb{Z}_{k^{\prime}}$ are the $k^{\prime}$ zeros of the Legendre polynomial of degree $k^{\prime}$ on $(-1,1)$, and

$$
w_{i}=\int_{-1}^{1} l_{i}(x) d x \quad \text { with } \quad l_{i}(x)=\prod_{j \neq i} \frac{x-u_{j}}{u_{i}-u_{j}}, \quad i \in \mathbb{Z}_{k^{\prime}} .
$$

Remark: We set $h(x ; \xi)=0$ if $\xi \in\left[t_{J-1}^{\prime}, t_{J+1}^{\prime}\right)$ when $x_{1} \leq 1-x_{1}$ or if $\xi \in\left[t_{n-1}^{\prime}, t_{n+1}^{\prime}\right)$ when $x_{1}>1-x_{1}$.

We need to evaluate $H\left(u_{e}\right), e \in \mathbb{Z}_{k^{\prime}}$. To this end, in turn we shall need to compute $h\left(x ; y_{i e}\right)$, where

$$
y_{i e}=\bar{t}_{i}^{-} u_{e}+\bar{t}_{i}^{+}
$$

Now

$$
\begin{aligned}
h\left(x ; y_{i e}\right) & =\int_{c}^{d-y_{i e}} K\left(x ;\left(y_{i e}, \eta\right)\right) f\left(y_{i e}, \eta\right) \mathrm{d} \eta \\
& :=\int_{c}^{d^{\prime}} g\left(x ;\left(y_{i e}, \eta\right)\right) \mathrm{d} \eta
\end{aligned}
$$

where

$$
g\left(x ;\left(y_{i e}, \eta\right)\right)=K\left(x ;\left(y_{i e}, \eta\right)\right) f\left(y_{i e}, \eta\right)
$$

We use exactly the same idea as to compute $I$ to compute $h\left(x ; y_{i e}\right)$. Denote the quadrature value of $h\left(x ; y_{i e}\right)$ by $h_{m}\left(x ; y_{i e}\right)$. Let $\triangle_{c}^{d^{\prime}}:=\left\{c=s_{1}<s_{2}<\cdots<s_{m_{2}}\right\}$ obtained similarly as we obtain $\triangle_{a}^{b}$, with $x_{2}$ as the shifting parameter. Then

$$
h\left(x ; y_{i e}\right):=\sum_{j=0}^{m_{2}-1} \int_{s_{j}}^{s_{j+1}} g\left(x ;\left(y_{i e}, \xi\right)\right) \mathrm{d} \xi=\int_{-1}^{1} G(\xi) \mathrm{d} \xi,
$$

with,

$$
G(\xi)=\sum_{j=0}^{m_{2}-1} \bar{s}_{j}^{-} g\left(x ;\left(y_{i e}, \bar{s}_{j}^{-} \xi+\bar{s}_{j}^{+}\right)\right)
$$

Again, using Gaussian quadrature method with the interpolating polynomial of degree $k^{\prime}$, we obtain

$$
h_{m}\left(x ; y_{i e}\right)=\sum_{j \in \mathbb{Z}_{k^{\prime}}} w_{j} G\left(u_{j}\right)
$$

where, $G\left(u_{e}\right), e \in \mathbb{Z}_{k^{\prime}}$ are computed by (4.40).

Remark: We set $g\left(x ;\left(y_{i e}, \xi\right)\right)=0$ if $\xi \in\left[s_{J-1}^{\prime}, s_{J+1}^{\prime}\right)$ when $x_{2} \leq 1-x_{2}$ or if $\xi \in\left[s_{n-1}^{\prime}, s_{n+1}^{\prime}\right)$ 
when $x_{2}>1-x_{2}$, where, $\triangle_{s^{\prime}}^{y_{2}}:=\left\{s_{j}^{\prime}: j \in \mathbb{Z}_{n+J+1}\right\}$ is similarly obtained as $\triangle_{t^{\prime}}^{y_{1}}$.

The numerical quadrature rule proposed here is extremely efficient, in the sense that not only it can automatically adjust the degree of fineness for the mesh regarding different domains of integration, but also use less and less grid points to compute the quadrature value when the domain is getting smaller and smaller. Nevertheless, in all cases it gives exactly same accuracies. We next show that the numerical integral $I_{m}$ computed by (4.36) for the weakly singular double integral $I$ in (4.22) is of order $2 k$, i.e.,

$$
\left|I_{m}-I\right|=\mathrm{O}\left(\delta_{m}{ }^{2 k}\right)
$$

where

$$
\delta_{m}:=\min \left(\frac{1}{2 m}, \max (b-a, d-c)\right) .
$$

\subsection{Error Analysis of the Adaptive Quadrature Rule}

Let us first recall for $F \in C^{2 k}(0,1)$,

- The $k$-point Gauss-Legendre rule:

$$
\int_{-1}^{1} F(x) d x=\sum_{i \in \mathbb{Z}_{k}} w_{i} F\left(u_{i}\right)+C_{k} F^{(2 k)}\left(\epsilon_{c}\right),
$$

where $\epsilon_{c}$ is some number in $(-1,1), C_{k}$ is a constant independent of $F,\left\{u_{i}\right\}, i \in \mathbb{Z}_{k}$ are $k$ zeros of the Legendre polynomial of degree $k$ on $(-1,1)$, and

$$
w_{i}=\int_{-1}^{1} l_{i}(x) d x \quad \text { with } \quad l_{i}(x)=\prod_{j \neq i} \frac{x-u_{j}}{u_{i}-u_{j}}, \quad i \in \mathbb{Z}_{k} .
$$

Next we establish a composite $k$-point Gauss-Legendre rule for singular integrals with $m$ non-uniform subdivisions (see $[\mathrm{KX}]$ for more related results).

Lemma 4.7 Suppose that $f$ defined on $(0,1)$ with

$$
\left|f^{(2 k)}(x)\right| \leq C x^{-\alpha-2 k}, \quad \text { for all } x \in(0,1) \text { and some } \alpha \in(0,1) \text {. }
$$


Yi Wang

Then, for any given integer $m>0$, there holds

$$
\int_{0}^{1} f(x) d x=\sum_{i \in \mathbb{Z}_{k}} w_{i}\left(\sum_{j=1}^{m-1} \bar{x}_{j}^{-} f\left(\bar{x}_{j}^{-} u_{i}+\bar{x}_{j}^{+}\right)\right)+O\left(\frac{1}{m^{2 k}}\right),
$$

where

$$
x_{j}=\left(\frac{j}{m}\right)^{q}, \quad q=\frac{2 k+1}{1-\alpha},
$$

for $j \in \mathbb{Z}_{m+1}$.

Proof: It follows from (4.44) that $|f(x)| \leq C x^{-\alpha}$ for $x \in(0,1)$, and thus we have

$$
\left|\int_{0}^{x_{1}} f(x) d x\right| \leq C x_{1}^{1-\alpha}=C \frac{1}{m^{2 k+1}} .
$$

Since

$$
\int_{0}^{1} f(x) d x=\int_{0}^{x_{1}} f(x) d x+\int_{x_{1}}^{1} f(x) d x
$$

and

$$
\int_{x_{1}}^{1} f(x)=\sum_{j=1}^{m-1} \int_{x_{j}}^{x_{j+1}} f(x) d x=\int_{-1}^{1} F(x) d x
$$

where

$$
F(x)=\sum_{j=1}^{m-1} \bar{x}_{j}^{-} \cdot f\left(\bar{x}_{j}^{-} x+\bar{x}_{j}^{+}\right),
$$

Hence, applying the $k$-point rule (4.43) to (4.48), yields

$$
\int_{x_{1}}^{1} f(x) d x=\int_{-1}^{1} F(x) d x=\sum_{i \in \mathbb{Z}_{k}} w_{i} F\left(u_{i}\right)+C_{k} F^{(2 k)}\left(\epsilon_{c}\right) .
$$

Note the first term on the right side of (4.50) is exactly the first term on the right side of (4.45), therefore it suffices to verify that the second term on the right side of (4.50) is of $O\left(\frac{1}{m^{2 k}}\right)$. From $(4.49)$,

$$
F^{(2 k)}(x)=\sum_{j=1}^{m-1}\left(\bar{x}_{j}^{-}\right)^{2 k+1} f^{(2 k)}\left(\bar{x}_{j}^{-} x+\bar{x}_{j}^{+}\right)
$$


and thus, by condition (4.44) on $f^{(2 k)}$,

$$
\begin{aligned}
\left|F^{(2 k)}\left(\epsilon_{c}\right)\right| & \leq \sum_{j=1}^{m-1}\left(\bar{x}_{j}^{-}\right)^{2 k+1}\left|f^{(2 k)}\left(\bar{x}_{j}^{-} \epsilon_{c}+\bar{x}_{j}^{+}\right)\right| \\
& \leq C \sum_{j=1}^{m-1}\left(2 \bar{x}_{j}^{-}\right)^{2 k+1}\left(x_{j}\right)^{-\alpha-2 k} \\
& =\frac{C}{m^{q(1-\alpha)}} \sum_{j=1}^{m-1}\left((j+1)^{q}-(j)^{q}\right)^{2 k+1}(j)^{-q(\alpha+2 k)} \\
& \leq \frac{C}{m^{q(1-\alpha)}} \sum_{j=1}^{m-1} q^{2 k+1} 2^{(q-1)(2 k+1)}(j)^{(q-1)(2 k+1)}(j)^{-q(\alpha+2 k)} \\
& =\frac{C}{m^{2 k+1}} \sum_{j=1}^{m-1} 1 \leq \frac{C}{m^{2 k}},
\end{aligned}
$$

where we have used the inequality

$$
(j+1)^{q}-(j)^{q}=q \xi^{q-1} \leq q(j+1)^{q-1} \leq q 2^{q-1}(j)^{q-1}
$$

with some $\xi \in(j, j+1)$ and $q \geq 1$. Combining (4.47), (4.46), (4.50) and (4.51), we obtain (4.45).

Theorem 4.8 Suppose that $f$ defined on $(0,1) \times(0,1)$ satisfies

$$
\left|\frac{\partial^{2 k} f}{\partial x^{2 k}}(x, y)\right| \leq C x^{-\alpha-2 k}, \quad\left|\frac{\partial^{2 k} f}{\partial y^{2 k}}(x, y)\right| \leq C y^{-\alpha-2 k}
$$

for all $(x, y) \in(0,1) \times(0,1)$ and some $\alpha \in(0,1)$. Let $\beta \in C^{2 k}[0,1]$ satisfying

$$
0 \leq \beta(x) \leq 1 \quad \text { on }(0,1)
$$

Then, for any given integer $m>0$, there holds

$$
\begin{aligned}
\int_{0}^{1} \int_{0}^{\beta(x)} f(x, y) d y d x= & \sum_{i \in \mathbb{Z}_{k}} w_{i}\left(\sum_{j=1}^{m-1} \bar{x}_{j}^{-} F_{i j}\right)+O\left(\frac{1}{m^{2 k}}\right), \\
& \text { with } F_{i j}=\sum_{\mu \in \mathbb{Z}_{k}} w_{\mu}\left(\sum_{\nu=1}^{m_{i j}-1} \bar{y}_{\nu}^{-} f\left(v_{i j}, \bar{y}_{\nu}^{-} u_{\mu}+\bar{y}_{\nu}^{+}\right)\right),
\end{aligned}
$$

where

$$
x_{j}=\left(\frac{j}{m}\right)^{q}, \quad j \in \mathbb{Z}_{m+1}, \quad q=\frac{2 k+1}{1-\alpha},
$$




$$
\begin{gathered}
v_{i j}=\bar{x}_{j}^{-} u_{i}+\bar{x}_{j}^{+}, \quad m_{i j}=\left\lceil\left(\beta\left(v_{i j}\right)\right)^{\frac{1}{q}} m\right\rceil, \\
y_{\nu}=\left(\frac{\nu}{m}\right)^{q}, \quad \nu \in \mathbb{Z}_{m_{i j}} \quad \text { and } \quad y_{m_{i j}}=\beta\left(v_{i j}\right) .
\end{gathered}
$$

Moreover the computational complexity $\mathcal{N}\left(I_{m}\right)$ is

$$
\mathcal{N}\left(I_{m}\right) \leq C m^{2}
$$

where, $\mathcal{N}\left(I_{m}\right)$ is the number of the point evaluations of the integrand $f$, and $I_{m}$ is the first term in (4.55), namely the numerical approximation of the integral.

Proof: Let

$$
F(x)=\int_{0}^{\beta(x)} f(x, y) d y
$$

Note

$F^{(2 k)}(x)=\int_{0}^{\beta(x)} \frac{\partial^{2 k} f}{\partial x^{2 k}}(x, y) d y+$ combination of lower partial derivatives of $f$ with respect to $x$.

By (4.53)-(4.54), we have that

$$
\left|F^{(2 k)}(x)\right| \leq C x^{-\alpha-2 k} \quad \text { for } x \in(0,1) .
$$

From Lemma 4.7,

$$
\int_{0}^{1} \int_{0}^{\beta(x)} f(x, y) d y d x=\int_{0}^{1} F(x) d x=\sum_{i \in \mathbb{Z}_{k}} w_{i}\left(\sum_{j=1}^{m-1} \bar{x}_{j}^{-} F\left(v_{i j}\right)\right)+O\left(\frac{1}{m^{2 k}}\right) .
$$

Each evaluation of $F\left(v_{i j}\right)$ requires another quadrature. Using Lemma 4.7 (4.45) again yields:

$$
F\left(v_{i j}\right)=\int_{0}^{\beta\left(v_{i j}\right)} f\left(v_{i j}, y\right) d y=F_{i j}+O\left(\frac{1}{m^{2 k}}\right)
$$

It remains to estimate the sum

$$
E_{i j}=\sum_{j=1}^{m-1} \bar{x}_{j}^{-} \frac{1}{m^{2 k}}
$$

While

$$
\begin{aligned}
\left|E_{i j}\right| & \leq 2 \sum_{j=1}^{m-1} 2 \bar{x}_{j}^{-} \frac{1}{m^{2 k}}=\frac{2}{m^{2 k}} \sum_{j=1}^{m-1} \frac{(j+1)^{q}-j^{q}}{m^{q}} \\
& \leq \frac{C}{m^{2 k}} \sum_{j=1}^{m-1} \frac{j^{q}}{m^{q}} \leq \frac{C}{m^{2 k}}
\end{aligned}
$$




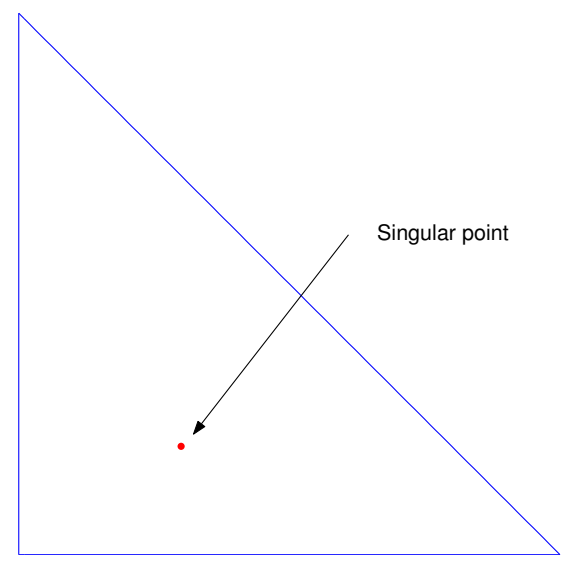

Figure 4.6: Domain of integration for Example 4.9

where we have used the inequality (4.52) again. Therefore, (4.55) follows from (4.57)-(4.59). In view of (4.55), it is clear that (4.94) holds.

We now present several numerical examples using above scheme. We shall consider $K(x, y)=$ $\frac{1}{x-y}$. The Gauss quadrature with interpolating polynomial of order $k=2$ is employed. Consequently, we choose $q=5$ for the non-uniform partition. The theoretical order of accuracy $(2 k=4)$ by this quadrature rule is verified in Example 4.9 and Example 4.10. The convergent rate is computed by $\log _{2} \frac{\left|I_{m}-I_{2 m}\right|}{\left|I_{2 m}-I_{4 m}\right|}$ in these two examples.

Example 4.9 Consider following integral

$$
I=\int_{0}^{1} \int_{0}^{1-x} \frac{x+y+1}{\sqrt{(x-0.3)^{2}+(y-0.2)^{2}}} \mathrm{~d} y \mathrm{~d} x
$$

In this example the singular point $(0.3,0.2)$ is inside the domain of integration-the unit triangle $\Omega$ (see Fig. 4.6). We tabulate our computation results in Table 4.1. It is clearly shown that the quadrature rule gives accurate results, and its order of convergence is also verified.

Example 4.10 Consider following integral

$$
I=\int_{0.5}^{1} \int_{0}^{1-x} \frac{x+y+1}{\sqrt{(x-0.3)^{2}+(y-0.2)^{2}}} \mathrm{~d} y \mathrm{~d} x .
$$




\begin{tabular}{c|c|c|c}
\hline $\mathrm{m}$ & Numerical $I_{m}$ & $\left|I_{m}-I_{2 m}\right|$ & Convergent rate \\
\hline 5 & 3.714414251744 & & \\
10 & 3.721811162863 & $7.3969 \mathrm{e}-03$ & \\
20 & 3.722373749843 & $5.6259 \mathrm{e}-04$ & 3.7168 \\
40 & 3.722412249476 & $3.8500 \mathrm{e}-05$ & 3.8692 \\
80 & 3.722414764040 & $2.5146 \mathrm{e}-06$ & 3.9365 \\
160 & 3.722414924162 & $1.6012 \mathrm{e}-07$ & 3.9731 \\
320 & 3.722414934272 & $1.0110 \mathrm{e}-08$ & 3.9853 \\
640 & 3.722414934906 & $6.3451 \mathrm{e}-10$ & 3.9940 \\
1280 & 3.722414934946 & $3.9740 \mathrm{e}-11$ & 3.9970 \\
2560 & 3.722414934948 & $2.4864 \mathrm{e}-12$ & 3.9985 \\
\hline
\end{tabular}

Table 4.1: Quadrature computing results for Example 4.9

Note, in this example, the singular point $(0.3,0.2)$ is outside the domain of integration (see Fig. 4.7). We tabulate the computing results in Table 4.2.

Example 4.11 In this example, we test if our quadrature rule is good enough in solving the actual integral equation (6.1), the linear system of equations are solved by standard Gaussian elimination method. No compression is assumed. The results are tabulated in Table 4.3. Order of convergence is computed by $\log _{2} \frac{\left\|u-u_{n}\right\|_{\infty}}{\left\|u-u_{n+1}\right\|_{\infty}}$, where $n$ stands for level number of the resolution, $u$ is the exact solution and $u_{n}$ is the approximation to $u$ in space $\mathbb{F}_{n}$; The theoretical convergent rate is 2 . We can see from the results that results by using our quadrature rule are very close to those obtained without quadrature rule.

Remark: The adaptive strategy of the proposed quadrature rule can be applied to any similar case.

Following theorem tells us that the adaptive strategy dramatically reduces the computational complexity for integration. 


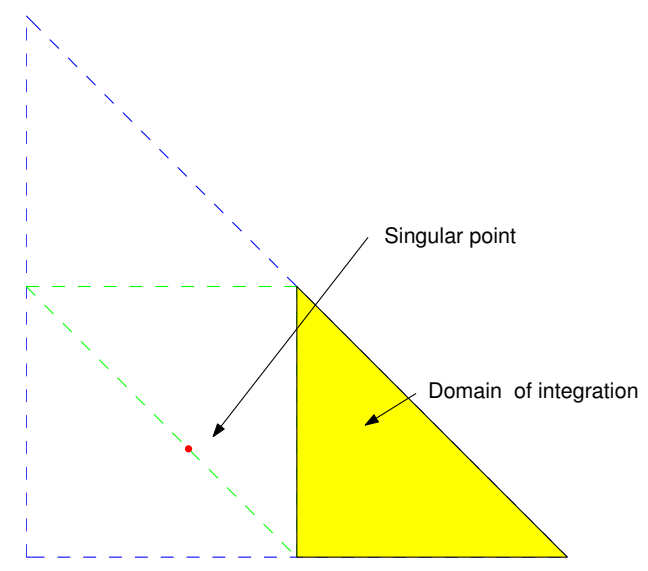

Figure 4.7: Domain of integration for Example 4.10

\begin{tabular}{c|c|c|c}
\hline $\mathrm{m}$ & Numerical $I_{m}$ & $\left|I_{m}-I_{2 m}\right|$ & Convergent rate \\
\hline 5 & 0.6337498001541 & & \\
10 & 0.6344202251264 & $6.7042 \mathrm{e}-04$ & \\
20 & 0.6344713062075 & $5.1081 \mathrm{e}-05$ & 3.7142 \\
40 & 0.6344745338648 & $3.2277 \mathrm{e}-06$ & 3.9842 \\
80 & 0.6344747360404 & $2.0218 \mathrm{e}-07$ & 3.9968 \\
160 & 0.6344747488410 & $1.2801 \mathrm{e}-08$ & 3.9813 \\
320 & 0.6344747496463 & $8.0531 \mathrm{e}-10$ & 3.9905 \\
640 & 0.6344747496970 & $5.0714 \mathrm{e}-11$ & 3.9891 \\
1280 & 0.6344747497002 & $3.1701 \mathrm{e}-12$ & 3.9998 \\
2560 & 0.6344747497004 & $1.9855 \mathrm{e}-13$ & 3.9970 \\
\hline
\end{tabular}

Table 4.2: Quadrature computing results for example 4.10 


\begin{tabular}{|c|c|c|c|c|c|}
\hline & \multicolumn{2}{|c|}{ without quadrature } & \multicolumn{3}{|c|}{ with quadrature } \\
$n$ & $L^{\infty}$-error & order & $\mathrm{m}$ & $L^{\infty}$-error & order \\
\hline 1 & $1.513187 \mathrm{e}-1$ & & 40 & $1.513184 \mathrm{e}-1$ & \\
2 & $3.599605 \mathrm{e}-2$ & 2.0717 & 40 & $3.599379 \mathrm{e}-2$ & 2.0718 \\
3 & $7.499082 \mathrm{e}-3$ & 2.2631 & 40 & $7.496564 \mathrm{e}-3$ & 2.2634 \\
4 & $1.849162 \mathrm{e}-3$ & 2.0198 & 40 & $1.850269 \mathrm{e}-3$ & 2.0185 \\
5 & $4.650610 \mathrm{e}-4$ & 1.9914 & 40 & $4.657219 \mathrm{e}-4$ & 1.9902 \\
\hline
\end{tabular}

Table 4.3: Comparison of uncompressed solutions by the wavelet collocation method, with and without numerical quadratures for matrix entries.

Theorem 4.12 In solving (2.2), if the basis functions of the approximation space are chosen as constructed in Chapter 3, and if the adaptive quadrature rule proposed in this section is employed, the complexity to compute all matrix entries in the discrete linear system is:

$$
\mathcal{N}\left(I_{m}\right) \leq C f(n) \log f(n) m^{2},
$$

where, $\mathcal{N}\left(I_{m}\right)$ is the number of function evaluations of integrands, $f(n)$ is the dimension of the approximation space, and $n$ is a resolution level of the approximation space.

Proof: The proof is done by direct counting. For any row $i^{\prime} j^{\prime},\left(i^{\prime}, j^{\prime}\right) \in \mathbb{U}_{n}$, in view of $(2.19)$, each entry in this row is computed through a $w_{i j}$, for $(i, j) \in \mathbb{U}_{n}$. We divide the elements in row $i^{\prime} j^{\prime}$ into $(n+1)$ subgroups according to their $i$ value. Namely, the elements of row $i^{\prime} j^{\prime}$ is divided into subgroups

$$
\begin{array}{ll}
K_{i^{\prime} j^{\prime}, 0 j}, & j \in \mathbb{Z}_{w(0)}, \\
K_{i^{\prime} j^{\prime}, 1 j}, & j \in \mathbb{Z}_{w(1)}, \\
\vdots & \vdots \\
K_{i^{\prime} j^{\prime}, n j}, & j \in \mathbb{Z}_{w(n)} .
\end{array}
$$

When $i=0, w_{0 j}, j \in \mathbb{Z}_{w(0)}$ are all supported on $\Omega$. There are $w(0)$ elements to be evaluated through integration, and each integration needs $\mathrm{Cm}^{2}$ function evaluations by Theorem 4.8. Hence the number of function evaluations is

$$
\mathcal{N}_{0}\left(I_{m}\right)=C w(0) m^{2}
$$


When $i=1, w_{1 j}, j \in \mathbb{Z}_{w(1)}$ are all supported on $\Omega$. The number of function evaluations is $($ note $w(1)=r)$

$$
\mathcal{N}_{1}\left(I_{m}\right)=\text { C.w }(1) m^{2}=\text { C.rm } m^{2}
$$

For $1<i \leq n$, since the supports of basis functions of $\mathbb{W}_{n}$ safisfies (2.7) and (2.8), then the number of function evaluations of the integrand is

$$
\mathcal{N}_{i}\left(I_{m}\right)=\text { C.rm }{ }^{2}
$$

Thus,

$$
\begin{aligned}
\mathcal{N}\left(I_{m}\right) & =f(n)\left(\mathcal{N}_{0}\left(I_{m}\right)+\mathcal{N}_{1}\left(I_{m}\right)+\cdots+\mathcal{N}_{n}\left(I_{m}\right)\right) \\
& =f(n)\left(C w(0) m^{2}+C r m^{2}+C r m^{2}+\ldots+C r m^{2}\right) \\
& =f(n)\left(C w(0) m^{2}+n \cdot C r m^{2}\right) \\
& \leq C f(n) \log f(n) m^{2} .
\end{aligned}
$$

\subsection{Compression Schemes-Distance Computing}

The matrix $\mathbf{K}_{\mathbf{n}}$ in general is non-symmetric and numerical sparse. [CMX3] gives following theoretical truncation criterion.

Let $\tilde{\mathbf{K}}_{n}:=\left[\tilde{K}_{i^{\prime} j^{\prime}, i j}:\left(i^{\prime}, j^{\prime}\right),(i, j) \in \mathbb{U}_{n}\right]$ be the truncation matrix whose entries are defined in terms of a matrix truncation parameter $\epsilon_{i^{\prime} i}$ by

$$
\tilde{K}_{i^{\prime} j^{\prime}, i j}:= \begin{cases}K_{i^{\prime} j^{\prime}, i j} & \text { if } \operatorname{dist}\left(\hat{S}_{i^{\prime} j^{\prime}}, S_{i j}\right) \leq \epsilon_{i^{\prime} i} \\ 0 & \text { otherwise }\end{cases}
$$

where $\operatorname{dist}\left(S_{i^{\prime} j^{\prime}}, S_{i, j}\right)$ denotes the geometric distance between the support $\hat{S}_{i^{\prime} j^{\prime}}$ of a collocation functional $\ell_{i^{\prime} j^{\prime}}$ and the support $S_{i j}$ of a wavelet function $w_{i j}$. The truncation parameter $\epsilon_{i^{\prime} i}$ is chosen such that for some positive constants $a$ and $\nu>1$,

$$
\epsilon_{i^{\prime} i}=\max \left\{a \mu^{\left[-i+b^{\prime}\left(n-i^{\prime}\right)\right] / 2}, \nu\left(d_{i}+d_{i^{\prime}}\right)\right\} \quad i, i^{\prime} \in \mathbb{Z}_{n+1},
$$

where,

$$
\frac{k-\sigma^{\prime}}{2 k-\sigma^{\prime}}<b^{\prime} \leq 1, \quad \text { with } 0<\sigma^{\prime}<2-\sigma .
$$




\begin{tabular}{|c|c|c|c|c|c|c|c|c|}
\hline strategies & \multicolumn{7}{|c|}{ Level $n$} \\
\hline & 2 & 3 & 4 & 5 & 6 & 7 & 8 & 9 \\
\hline strategy $1(\nu=1)$ & 1.0000 & 0.9780 & 0.6766 & 0.3172 & 0.1198 & 0.0408 & 0.0135 & 0.0045 \\
\hline strategy $2(\nu=1)$ & 1.0000 & 0.8945 & 0.5202 & 0.2276 & 0.0857 & 0.0302 & 0.0105 & 0.0037 \\
\hline strategy $3(\nu=1)$ & 1.0000 & 0.8857 & 0.4488 & 0.1699 & 0.0572 & 0.0189 & 0.0066 & 0.0025 \\
\hline $\begin{array}{c}\text { block truncation } \\
\left(p_{i^{\prime} i}=\sqrt{3}\right)\end{array}$ & 1.0000 & 0.7583 & 0.4166 & 0.1810 & 0.0677 & 0.0230 & 0.0073 & 0.0022 \\
\hline
\end{tabular}

Table 4.4: Comparison of compression rates of different strategies

$d_{i}:=O\left(\mu^{-(i / 2)}\right)$ is the diameter of $S_{i j}, j \in \mathbb{Z}_{w(i)} . n$ is the level number of the approximation space. Note the truncating parameter $\epsilon_{i^{\prime} i}$ (4.64) changes as $i, i^{\prime}$ change. We present here several different practical strategies. Three strategies are investigated in this section. For strategy 1 we choose

$$
\epsilon_{i^{\prime} i}=\nu\left(d_{i^{\prime}}+d_{i}\right)
$$

For strategy 2 we choose

$$
\epsilon_{i^{\prime} i}=\nu \max \left\{d_{i^{\prime}}, d_{i}\right\}
$$

For strategy 3 we choose

$$
\epsilon_{i^{\prime} i}=\nu \min \left\{d_{i^{\prime}}, d_{i}\right\}
$$

A comparison of compression rates of different strategies is depicted in Fig. 4.8, their actual numbers are also listed in Table 4.4 (We take $\nu=1$ for strategy 1,2,3). The compression rate is computed as the ratio of the number of nonzero entries in the compressed matrix $\tilde{\mathbf{K}}_{n}$ over the total number of entries of $\mathbf{K}_{n}$. Observe that the compression rate of strategy 2 is in between of those of strategy 1 and strategy 3 .

Moreover, we test these different strategies solving the integral equation (6.1) with $\nu=$ 1. In order to exclude other affecting factors, we use standard Gaussian elimination to solve the resulting system. All the matrix entries are computed exactly. The results are tabulated in Table 4.5, Table 4.6 and Table 4.7. Order of convergence is computed by $\log _{2} \frac{\left\|u-u_{n}\right\|_{\infty}}{\left\|u-u_{n+1}\right\|_{\infty}}$, where $n$ stands for level number of the resolution, $u$ is the exact solution and $u_{n}$ is an approximation to $u$ in space $\mathbb{F}_{n}$; The theoretical convergent rate is 2 . The 


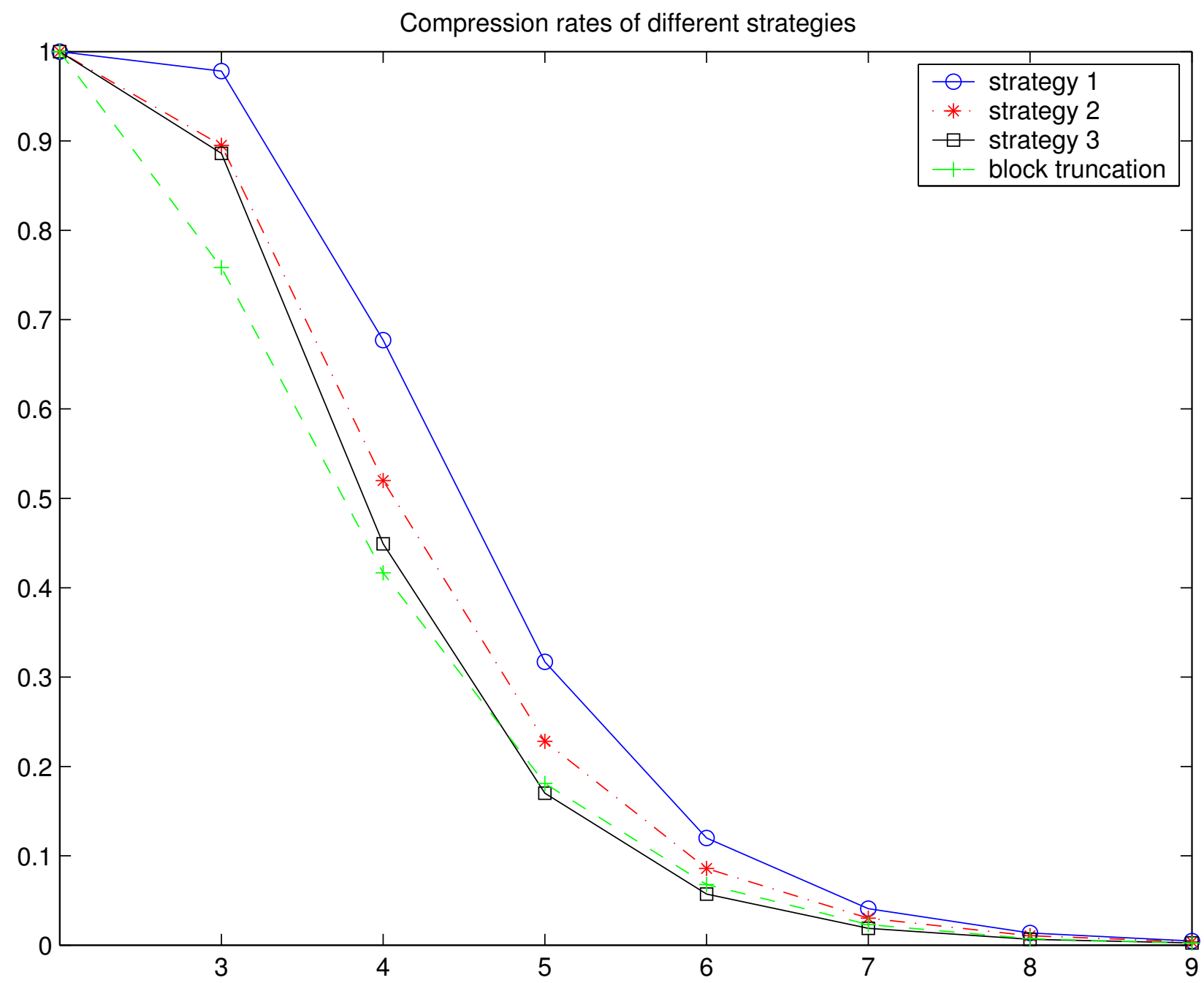

Figure 4.8: Compression rates of different strategies 


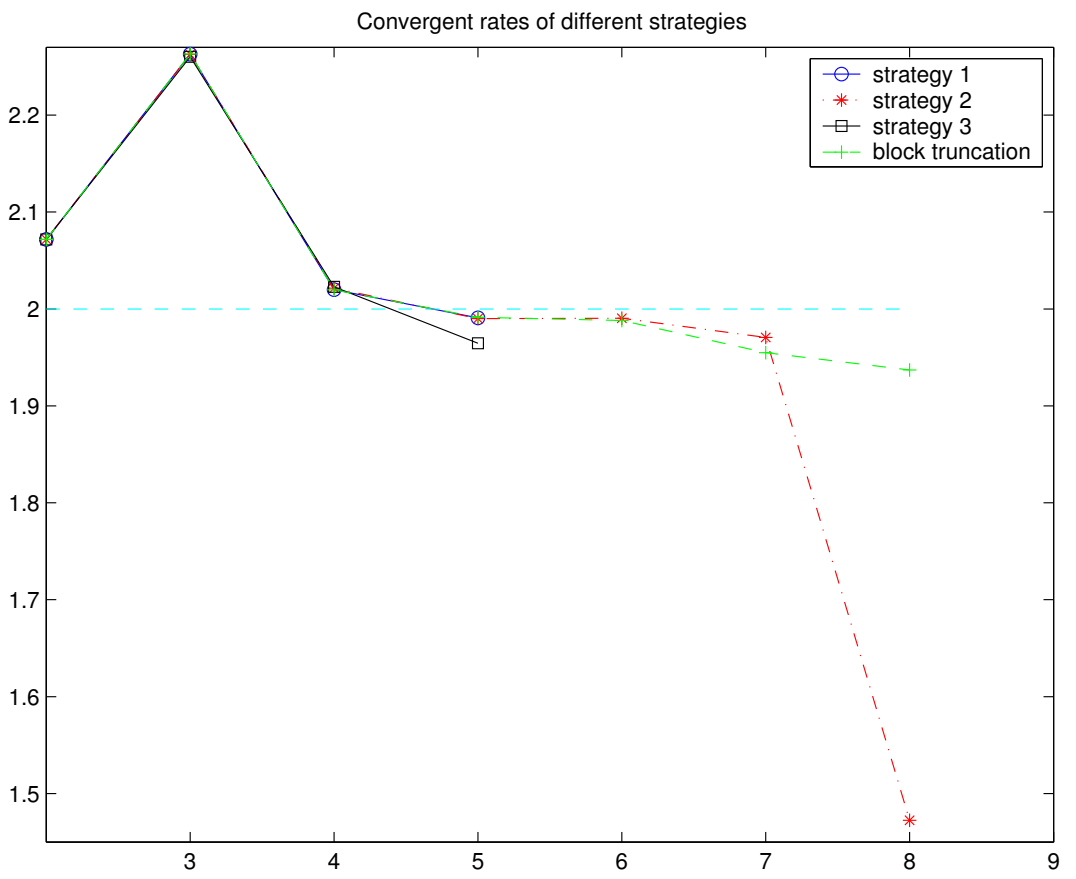

Figure 4.9: Convergent rates of different strategies

comparison of their convergent rates for different strategies is plotted in Figure 4.9. By comparing with the theoretical convergent rate 2, from Fig. 4.9 we can tell that strategy 1 is relatively conservative and strategy 3 is somewhat insufficient. While strategy 2 gives relatively satisfied results.

We further investigate computing results by choosing different parameter $\nu$. The results are tabulated in Table 4.8, Table 4.9 and Table 4.10. The convergent rates of strategy 3 with different $\nu$ are depicted in Figure 4.10, and the convergent rates of strategy 2 with different $\nu$ are depicted in Figure 4.11. From those tabulated results and the two figures Fig. 4.10 and Fig. 4.11, we see that for the adopted strategy $2, \nu=1$ gives satisfied results. While smaller $\nu$ doesn't give satisfied results.

The matrix $\mathbf{K}_{n}$ after compression is quite sparse when $n$ is relatively large (See Fig. 4.12, Fig. 4.13 and Fig. 4.14. These sparse matrices are computed for integral equation (6.1). Special sparse matrix storage is used when doing the actual computing. Not only this speeds up computing, but also this dramatically saves computer memory. In fact when $n$ 
Strategy 1: $\epsilon_{i^{\prime} i}=\nu\left(d_{i^{\prime}}+d_{i}\right)$

\begin{tabular}{|c|c|c|c|c|c|}
\hline & \multicolumn{2}{|c|}{ full collocation solution $u_{n}$} & \multicolumn{3}{|c|}{ compressed solution $\tilde{u}_{n}$} \\
$n$ & $L^{\infty}$-error & order & $L^{\infty}$-error & order & compression rate \\
\hline 1 & $1.513187 \mathrm{e}-1$ & & $1.513187 \mathrm{e}-1$ & & 1.000000 \\
2 & $3.599605 \mathrm{e}-2$ & 2.0717 & $3.599605 \mathrm{e}-2$ & 2.0717 & 1.000000 \\
3 & $7.499082 \mathrm{e}-3$ & 2.2631 & $7.499309 \mathrm{e}-3$ & 2.2630 & 0.978027 \\
4 & $1.849162 \mathrm{e}-3$ & 2.0198 & $1.849060 \mathrm{e}-3$ & 2.0200 & 0.676590 \\
5 & $4.650610 \mathrm{e}-4$ & 1.9914 & $4.652252 \mathrm{e}-4$ & 1.9908 & 0.317277 \\
\hline
\end{tabular}

Table 4.5: Comparison of solutions obtained by the full collocation method and by our compressed collocation method (strategy 1 with $\nu=1$ ).

Strategy 2: $\epsilon_{i^{\prime} i}=\nu \max \left\{d_{i^{\prime}}, d_{i}\right\}$

\begin{tabular}{|c|c|c|c|c|c|}
\hline & \multicolumn{2}{|c|}{ full collocation solution $u_{n}$} & \multicolumn{3}{|c|}{ compressed solution $\tilde{u}_{n}$} \\
$n$ & $L^{\infty}$-error & order & $L^{\infty}$-error & order & compression rate \\
\hline 1 & $1.513187 \mathrm{e}-1$ & & $1.513187 \mathrm{e}-1$ & & 1.000000 \\
2 & $3.599605 \mathrm{e}-2$ & 2.0717 & $3.599605 \mathrm{e}-2$ & 2.0717 & 1.000000 \\
3 & $7.499082 \mathrm{e}-3$ & 2.2631 & $7.501118 \mathrm{e}-3$ & 2.2627 & 0.894531 \\
4 & $1.849162 \mathrm{e}-3$ & 2.0198 & $1.847625 \mathrm{e}-3$ & 2.0214 & 0.520172 \\
5 & $4.650610 \mathrm{e}-4$ & 1.9914 & $4.650882 \mathrm{e}-4$ & 1.9901 & 0.227601 \\
\hline
\end{tabular}

Table 4.6: Comparison of solutions obtained by the full collocation method and by our compressed collocation method (strategy 2 with $\nu=1$ ). 
Strategy 3: $\epsilon_{i^{\prime} i}=\nu \min \left\{d_{i^{\prime}}, d_{i}\right\}$

\begin{tabular}{|c|c|c|c|c|c|}
\hline \multicolumn{3}{|c|}{ full collocation solution $u_{n}$} & \multicolumn{3}{|c|}{ compressed solution $\tilde{u}_{n}$} \\
$n$ & $L^{\infty}$-error & order & $L^{\infty}$-error & order & compression rate \\
\hline 1 & $1.513187 \mathrm{e}-1$ & & $1.513187 \mathrm{e}-1$ & & 1.000000 \\
2 & $3.599605 \mathrm{e}-2$ & 2.0717 & $3.599605 \mathrm{e}-2$ & 2.0717 & 1.000000 \\
3 & $7.499082 \mathrm{e}-3$ & 2.2631 & $7.514074 \mathrm{e}-3$ & 2.2602 & 0.885742 \\
4 & $1.849162 \mathrm{e}-3$ & 2.0198 & $1.848971 \mathrm{e}-3$ & 2.0229 & 0.448761 \\
5 & $4.650610 \mathrm{e}-4$ & 1.9914 & $4.737033 \mathrm{e}-4$ & 1.9647 & 0.169880 \\
\hline
\end{tabular}

Table 4.7: Comparison of solutions obtained by the full collocation method and by our compressed collocation method (strategy 3 with $\nu=1$ ).

Strategy 3: $\epsilon_{i^{\prime} i}=\nu \min \left\{d_{i^{\prime}}, d_{i}\right\}$

\begin{tabular}{|c|c|c|c|c|c|c|}
\hline \multirow[b]{2}{*}{$\mathrm{n}$} & \multicolumn{3}{|c|}{$\nu=1$} & \multicolumn{3}{|c|}{$\nu=0.8$} \\
\hline & $L_{\infty}$ error & order & comp. rate & $L_{\infty}$ error & order & comp. rate \\
\hline 1 & $1.513187 \mathrm{e}-1$ & & 1.0000 & $1.513187 \mathrm{e}-1$ & & 1.0000 \\
\hline 2 & $3.599605 \mathrm{e}-2$ & 2.0717 & 1.0000 & $3.599605 \mathrm{e}-2$ & 2.0717 & 1.0000 \\
\hline 3 & $7.514074 \mathrm{e}-3$ & 2.2602 & 0.885742 & 7.406683e-3 & 2.2809 & 0.674805 \\
\hline 4 & $1.848971 \mathrm{e}-3$ & 2.0229 & 0.448761 & $1.879626 \mathrm{e}-3$ & 1.9784 & 0.301544 \\
\hline 5 & $4.737033 \mathrm{e}-4$ & 1.9647 & 0.169880 & $6.657693 \mathrm{e}-4$ & 1.4974 & 0.111790 \\
\hline
\end{tabular}

Table 4.8: Comparison of solutions obtained from strategy 3 with different $\nu$

Strategy 2: $\epsilon_{i^{\prime} i}=\nu \max \left\{d_{i^{\prime}}, d_{i}\right\}$

\begin{tabular}{|c|c|c|c|c|c|c|}
\hline \multirow[b]{2}{*}{$\mathrm{n}$} & \multicolumn{3}{|c|}{$\nu=1$} & \multicolumn{3}{|c|}{$\nu=0.8$} \\
\hline & $L_{\infty}$ error & order & comp. rate & $L_{\infty}$ error & order & comp. rate \\
\hline 1 & $1.513187 \mathrm{e}-1$ & & 1.000000 & $1.513187 \mathrm{e}-1$ & & 1.000000 \\
\hline 2 & $3.599605 \mathrm{e}-2$ & 2.0717 & 1.000000 & $3.599605 \mathrm{e}-2$ & 2.0717 & 1.000000 \\
\hline 3 & 7.501118e-3 & 2.2627 & 0.894531 & 7.494318e-3 & 2.2640 & 0.753906 \\
\hline 4 & $1.847625 \mathrm{e}-3$ & 2.0214 & 0.520172 & $1.850194 \mathrm{e}-3$ & 2.0181 & 0.407013 \\
\hline 5 & $4.650882 \mathrm{e}-4$ & 1.9901 & 0.227601 & $4.689467 \mathrm{e}-4$ & 1.9802 & 0.174738 \\
\hline
\end{tabular}

Table 4.9: Comparison of solutions obtained from strategy 2 with different $\nu$ 
Strategy 2: $\epsilon_{i^{\prime} i}=\nu \max \left\{d_{i^{\prime}}, d_{i}\right\}$

\begin{tabular}{|c|c|c|c|c|c|c|}
\hline & \multicolumn{3}{|c|}{$\nu=0.6$} \\
\hline $\mathrm{n}$ & $L_{\infty}$ error & order & comp. rate & $L_{\infty}$ error & order & comp. rate \\
\hline 1 & $1.513187 \mathrm{e}-1$ & & 1.0000 & $1.513187 \mathrm{e}-1$ & & 1.0000 \\
2 & $3.599605 \mathrm{e}-2$ & 2.0717 & 1.0000 & $3.599605 \mathrm{e}-2$ & 2.0717 & 1.0000 \\
3 & $7.510530 \mathrm{e}-3$ & 2.2609 & 0.712158 & $7.409970 \mathrm{e}-3$ & 2.2803 & 0.641846 \\
4 & $1.855561 \mathrm{e}-3$ & 2.0171 & 0.360596 & $1.887554 \mathrm{e}-3$ & 1.9729 & 0.297562 \\
5 & $4.784063 \mathrm{e}-4$ & 1.9555 & 0.148113 & $5.564827 \mathrm{e}-4$ & 1.7621 & 0.118184 \\
\hline
\end{tabular}

Table 4.10: Comparison of solutions obtained from strategy 2 with different $\nu$

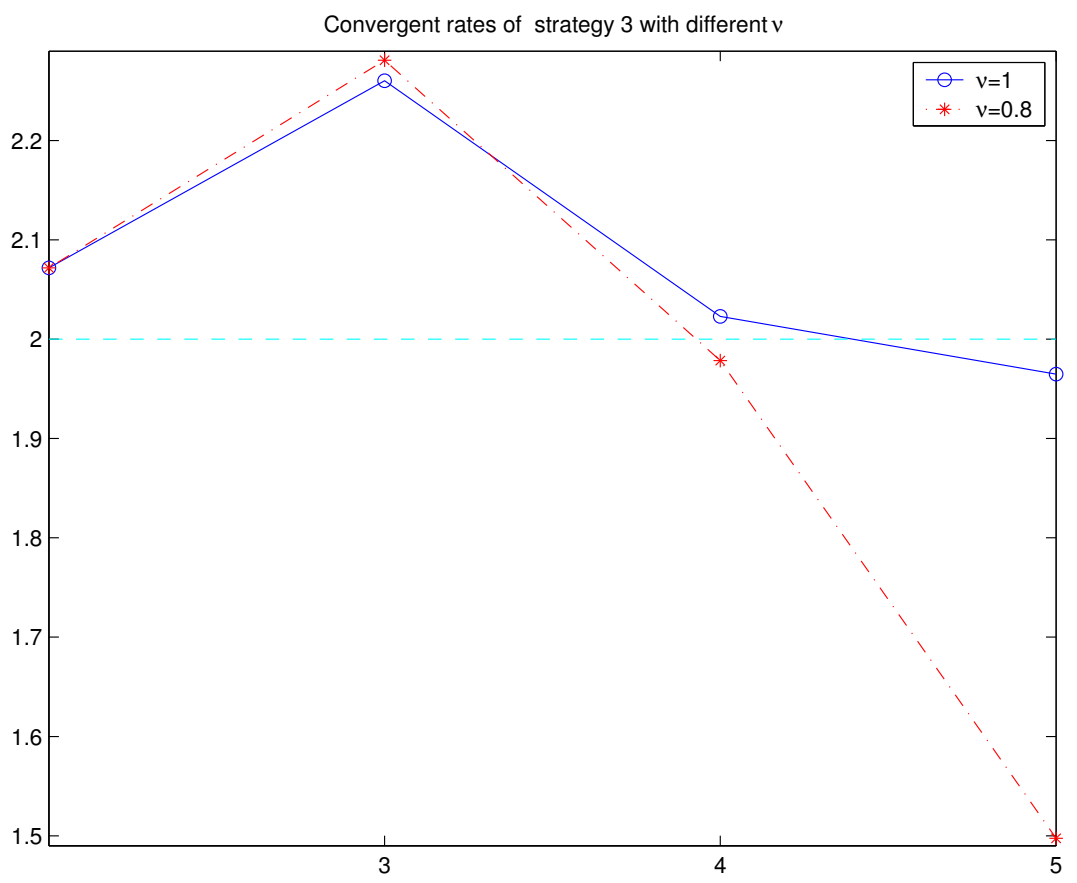

Figure 4.10: Convergent rates of strategy 3 with different $\nu$ 


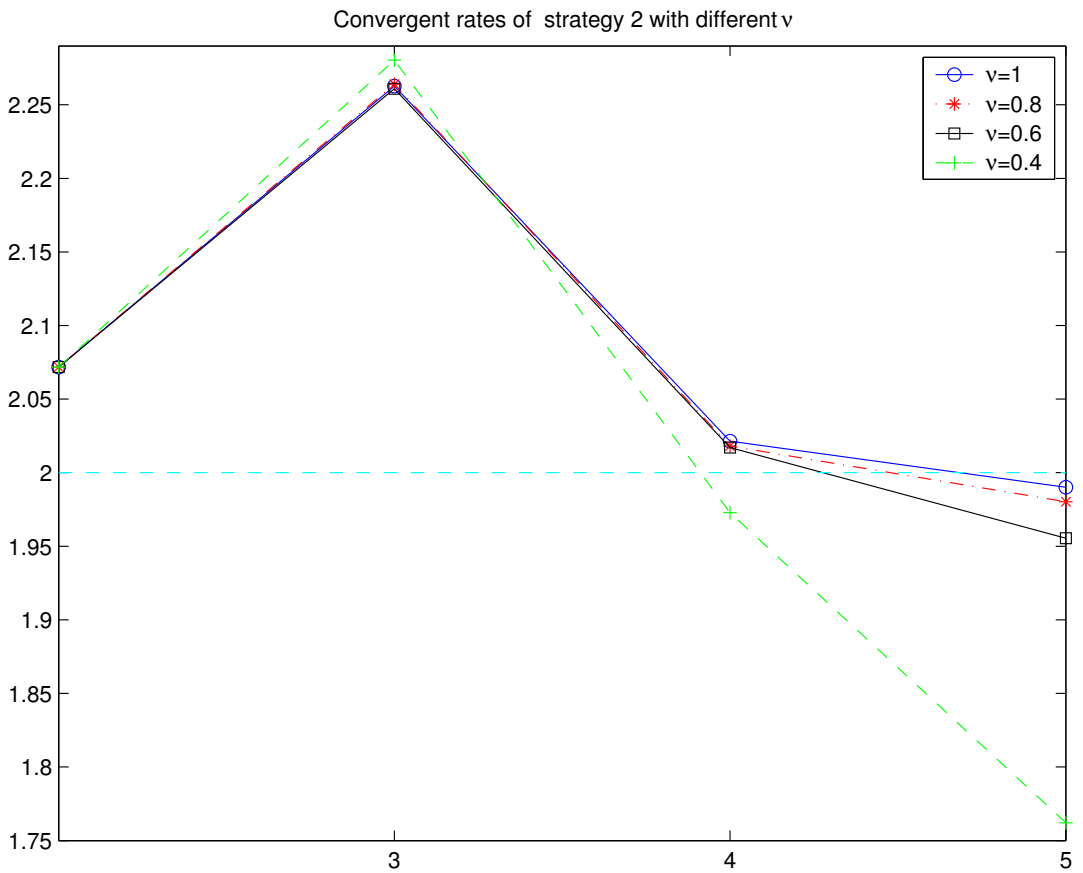

Figure 4.11: Convergent rates of strategy 2 with different $\nu$

is relatively large, we are forced to use the sparse storage scheme to store the matrix (On our computer cluster.math.wvu.edu, with AMD Athlon(tm) MP Processor 1600+ and 1 Gigabyte of RAM, if serial codes are used, we have to use sparse storage when $n>5$ ).

For comparison of the compressed matrix $\tilde{\mathbf{K}}_{n}$ with the full matrix $\mathbf{K}_{n}$, in Fig. 4.15, we plot both $\tilde{\mathbf{K}}_{3}$ and $\mathbf{K}_{3}$. Note in Fig. 4.15, for viewing effect, we use the absolute values of entries of the matrix $\tilde{\mathbf{K}}_{3}$ and $\mathbf{K}_{3}$.

Next several theorems claim that if the constructed wavelets and collocation functionals on the unit triangle are employed to solve (2.2) on $\Omega$, then similar results as in [CMX3] continue to hold.

Theorem 4.13 If the constructed wavelets and collocation functionals on the unit triangle in Chapter 3 are employed to solve (2.2) on the unit triangle $\Omega$, and if the truncation parameters $\epsilon_{i^{\prime} i}^{n}, i, i^{\prime} \in \mathbb{Z}_{n+1}$ are chosen as (4.64) then there exists a positive constant $c$ and a positive integer $M$ such that when $n \geq M$ and for $x \in \mathbb{F}_{n}$,

$$
\left\|\left(\mathcal{I}-\tilde{\mathcal{K}}_{n}\right) x\right\|_{\infty} \geq c\|x\|_{\infty}
$$




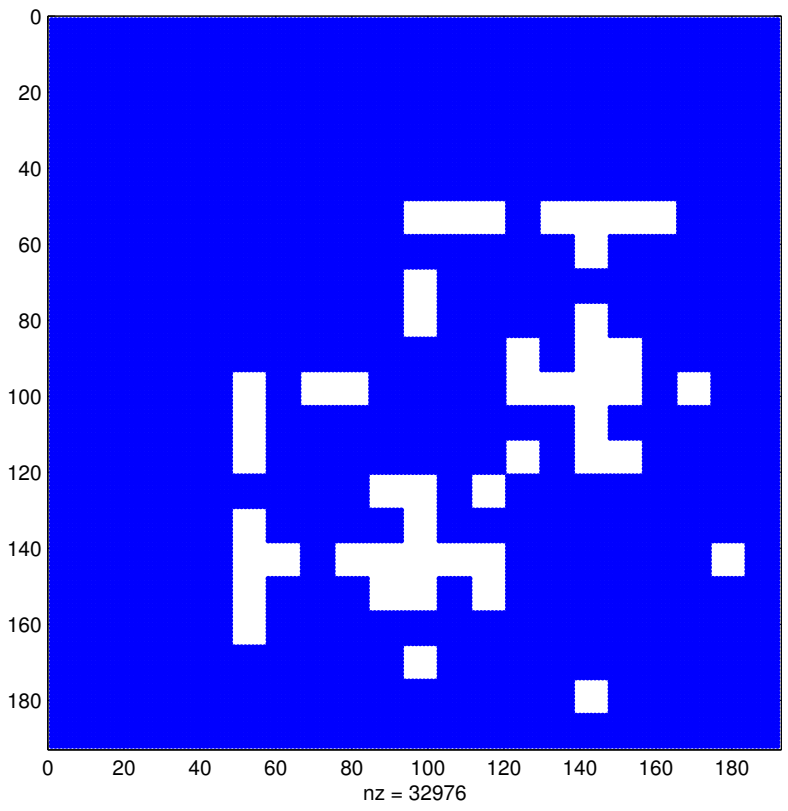

Figure 4.12: The sparse matrix $\mathbf{K}_{3}$ after compression

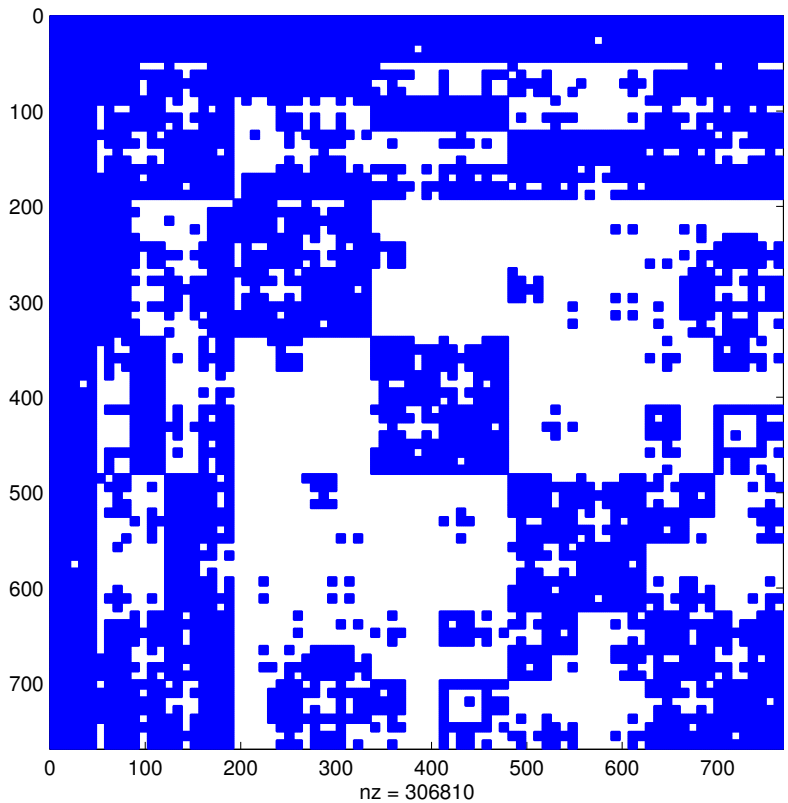

Figure 4.13: The sparse matrix $\mathbf{K}_{4}$ after compression 


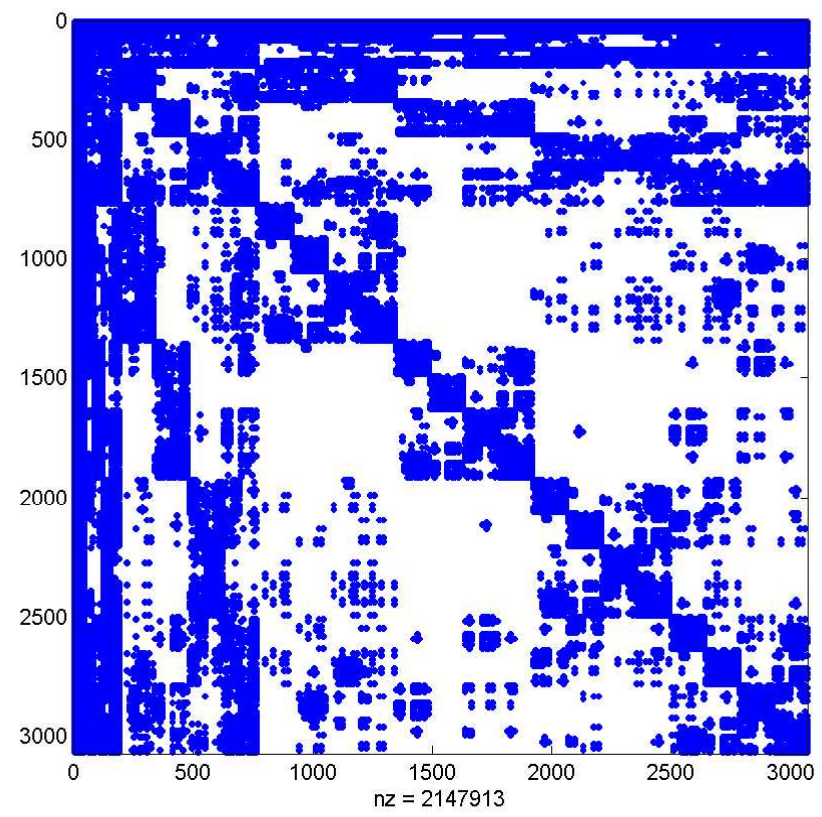

Figure 4.14: The sparse matrix $\mathbf{K}_{5}$ after compression

i.e., the compressed scheme (4.64) is stable, where, $\tilde{\mathcal{K}}_{n}: \mathbb{F}_{n} \mapsto \mathbb{F}_{n}$ is a linear operator relative to the basis $\left\{w_{i j}:(i, j) \in \mathbb{U}_{n}\right\}$ with $\mathbf{E}_{n}^{-1} \tilde{\mathbf{K}}_{n}$ as its matrix representation.

Proof: Since the constructed wavelet basis and collocation functionals have properties Lemma 3.1 through Lemma 3.7 and the kernel considered satisfying (4.23), the theorem follows from THEOREM 4.3 of [CMX3].

In Particular, this theorem allows for $n \geq M$,

$$
\left(\mathcal{I}-\tilde{\mathcal{K}}_{n}\right) \tilde{u}_{n}=\mathcal{P}_{n} f
$$

has a unique solution given by $\tilde{u}_{n}=\sum_{(i, j) \in \mathbb{U}_{n}} \tilde{u}_{i j} w_{i j}$.

Theorem 4.14 Use the constructed wavelets and collocation functionals on the unit triangle in Chapter 3 to solve (2.2) on $\Omega$. Let $\epsilon_{i^{\prime} i}^{n}, i, i^{\prime} \in \mathbb{Z}_{n+1}$, be chosen as in (4.64). Then there exists a positive constant $c$ and a positive integer $M$ such that for all $n \geq M$,

$$
\left\|u-\tilde{u}_{n}\right\|_{\infty} \leq c f(n)^{-k / 2}(\log f(n))\|u\|_{k, \infty} .
$$



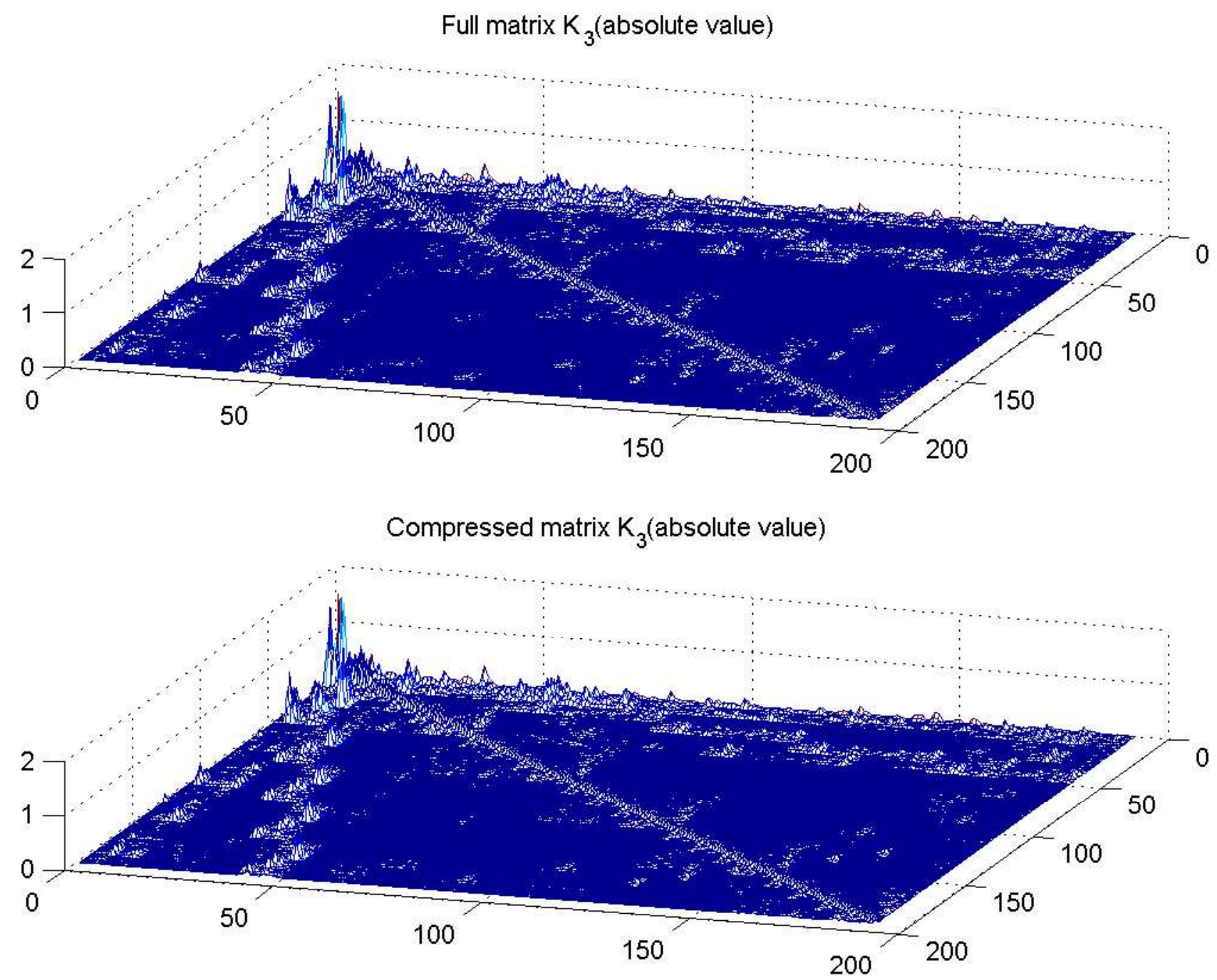

Figure 4.15: The Full matrix $\mathbf{K}_{3}$ and the compressed matrix $\tilde{\mathbf{K}}_{3}$ 
Proof: Since the constructed wavelet basis and collocation functionals have properties Lemma 3.1 through Lemma 3.8 and the kernel considered satisfying (4.23), the theorem follows from THEOREM 4.4 of [CMX3].

Theorem 4.15 If the conditions in THEOREM 4.13 hold, then there exists a positive constant $c$ such that the condition number of the matrix $\tilde{\mathbf{A}}_{n}:=\mathbf{E}_{n}-\tilde{\mathbf{K}}_{n}$ satisfies

$$
\operatorname{cond}_{\infty}\left(\tilde{\mathbf{A}}_{n}\right) \leq c \log ^{2}(f(n))
$$

where $\operatorname{cond}_{\infty}(\mathbf{A})$ denotes the condition number of a matrix $\mathbf{A}$ in $\ell^{\infty}$ matrix norm.

Proof: Since the constructed wavelet basis and collocation functionals have properties Lemma 3.1 through Lemma 3.7 and the kernel considered satisfying (4.23), the theorem follows from THEOREM 4.5 of [CMX3].

Theorem 4.16 Use the constructed wavelets and collocation functionals on the unit triangle in Chapter 3 to solve (2.2) on $\Omega$. Let the truncation parameters $\epsilon_{i^{\prime} i}^{n}, i^{\prime}, i \in \mathbb{Z}_{n+1}$, be chosen as in (4.64). Then

$$
\mathcal{N}\left(\tilde{\mathbf{A}}_{n}\right)=\mathcal{O}(f(n) \log f(n))
$$

Proof: Since the constructed wavelet basis and collocation functionals have property Lemma 3.1 and property Lemma 3.4, the theorem follows from THEOREM 4.6 of [CMX3].

\subsection{Block Truncation Schemes}

We describe in this section a block truncation scheme. This idea is also studied by [CWX1] in their study of 1-D wavelet collocation methods. By this scheme, the actual geometric distance $\operatorname{dist}\left(\hat{S}_{i^{\prime} j^{\prime}}, S_{i j}\right)$ is not needed to determine if an element $K_{i^{\prime} j^{\prime}, i j},\left(i^{\prime}, j^{\prime}\right),(i, j) \in \mathbb{U}_{n}$ needs to be truncated. We first note

$$
S_{i j}=\phi_{\mathbf{e}}(\Omega)
$$


and

$$
\hat{S}_{i^{\prime} j^{\prime}}=\phi_{\mathbf{e}^{\prime}}(\Omega)
$$

$\hat{S}_{i^{\prime} j^{\prime}}$ and $S_{i j}$ are both right triangles transformed from the original unit triangle $\Omega$. For $S_{i j}$, denote its vertex of right angle by $P_{0}\left(x_{0}, y_{0}\right)$, and counterclockwisely, denote the other two vertices by $P_{1}\left(x_{1}, y_{1}\right)$ and $P_{2}\left(x_{2}, y_{2}\right)$ (see Fig. 4.4). It is not difficult to find the centroid of the $S_{i j}$ is at

$$
C=\left(x_{0}+\frac{x_{1}-x_{0}}{3}, y_{0}+\frac{x_{1}-x_{0}}{3}\right):=\left(C_{x}, C_{y}\right) .
$$

We assign a multi-index $\mathbf{q}:=\left(q_{1}, q_{2}\right)$ to $S_{i j}$, and $\mathbf{q}$ is determined by

$$
q_{1}=\left\lfloor\frac{C_{x}}{\mu^{(i-1) / 2}}\right\rfloor \quad \text { and } \quad q_{2}=\left\lfloor\frac{C_{y}}{\mu^{(i-1) / 2}}\right\rfloor .
$$

This can be written as

$$
\mathbf{q}=\gamma(\mathbf{e})
$$

where $\gamma$ is a function defined by $\gamma: \mathbb{Z}_{\mu}^{i-1} \mapsto \mathbb{Z}_{\mu^{(i-1) / 2}}^{2}$.

If $i \geq i^{\prime}$, let $\mathbf{e}=\left(e_{0} e_{1} \ldots e_{i-2}\right)$ and $\mathbf{e}^{\prime}=\left(e_{0}^{\prime} e_{1}^{\prime} \ldots e_{i^{\prime}-2}^{\prime}\right)$. Denote $\mathbf{e}_{c}=\left(e_{0} e_{1} \ldots e_{i^{\prime}-2}\right)$. We then have

$$
\mathbf{q}^{\prime}=\left(q_{1}^{\prime}, q_{2}^{\prime}\right)=\gamma\left(\mathbf{e}^{\prime}\right)
$$

and

$$
\mathbf{q}=\left(q_{1}, q_{2}\right)=\gamma\left(\mathbf{e}_{c}\right)
$$

If $i<i^{\prime}$, denote $\mathbf{e}_{c}^{\prime}=\left(e_{0}^{\prime} e_{1}^{\prime} \ldots e_{i-2}^{\prime}\right)$. We have

$$
\mathbf{q}^{\prime}=\left(q_{1}^{\prime}, q_{2}^{\prime}\right)=\gamma\left(\mathbf{e}_{c}^{\prime}\right)
$$

and

$$
\mathbf{q}=\left(q_{1}, q_{2}\right)=\gamma(\mathbf{e})
$$

Now define a block $\mathbf{K}_{\mathbf{q}^{\prime} \mathbf{q}}^{i^{\prime} i}$ by

$$
\mathbf{K}_{\mathbf{q}^{\prime} \mathbf{q}}^{i^{\prime} i}:=\left\{K_{i^{\prime} j^{\prime}, i j}: \mathbf{q}^{\prime}, \mathbf{q} \text { are determined by }(4.74)-(4.77)\right\}
$$

We have, in both cases, that

$$
\mathbf{K}_{i^{\prime} i}=\left[\mathbf{K}_{\mathbf{q}^{\prime} \mathbf{q}}^{i^{\prime}}: \mathbf{q}^{\prime}, \mathbf{q} \in \mathbb{Z}_{\mu^{\left(i_{0}-1\right) / 2}}^{2}\right]
$$




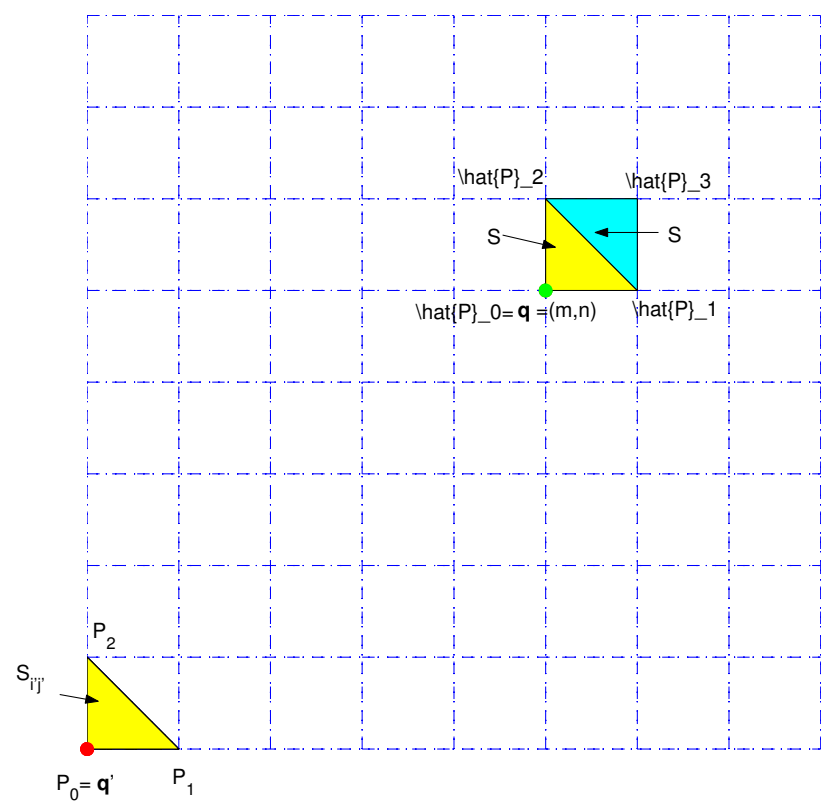

Figure 4.16: Distance of two triangles $S_{i^{\prime} j^{\prime}}$ and $S$

where $i_{0}:=\min \left\{i^{\prime}, i\right\}$. Figure 4.16 shows the idea of the block truncation scheme. If the support $\hat{S}_{i^{\prime} j^{\prime}}$ of the collocation functional $\ell_{i^{\prime} j^{\prime}}$ is in a finer level comparing with the support $S_{i j}$ of the wavelet function $w_{i j}$, then the support of the same level as that of the wavelet containing $\hat{S}_{i^{\prime} j^{\prime}}$ is considered when $\mathbf{q}^{\prime}$ is assigned. Similar idea is applied if the support $S_{i j}$ of the wavelet $w_{i j}$ is in a finer level. Denote $S=\phi_{\mathbf{e}_{c}}(\Omega)$ if $i \geq i^{\prime}$ and $S=\phi_{\mathbf{e}_{c}^{\prime}}(\Omega)$ if $i<i^{\prime}$. We have following lemma:

Lemma 4.17 Assume that $K_{i^{\prime} j^{\prime}, i j}$ is an entry of $\mathbf{K}_{\mathbf{q}^{\prime} \mathbf{q}}^{i^{\prime} i}$. Then, for $i \geq i^{\prime}$,

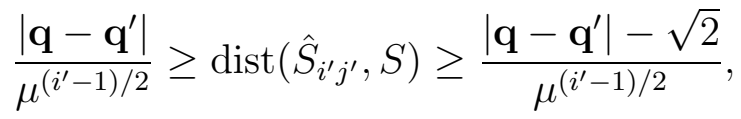

and for $i<i^{\prime}$,

$$
\frac{\left|\mathbf{q}-\mathbf{q}^{\prime}\right|}{\mu^{(i-1) / 2}} \geq \operatorname{dist}\left(S_{i j}, S\right) \geq \frac{\left|\mathbf{q}-\mathbf{q}^{\prime}\right|-\sqrt{2}}{\mu^{(i-1) / 2}} .
$$

Proof: We present a proof for the case $i \geq i^{\prime}$. The proof for the case $i<i^{\prime}$ is similar. Without loss of generality, assume vertices of $\hat{S}_{i^{\prime} j^{\prime}}$ are $P_{i}, i \in \mathbb{Z}_{3}$ with $P_{0}=(0,0)$ (see Figure 4.16). Note $\hat{S}_{i^{\prime} j^{\prime}}$ is a right triangle with two equal sides. Each side is of length 1 unit, where the unit is $\frac{1}{\mu^{\left(i^{\prime}-1\right) / 2}}$. Following we only use the number of units to describe the length. With 
this assumption, we have $\mathbf{q}^{\prime}=(0,0)$. Assume $\mathbf{q}=(m, n)$, where $m, n$ are integers. Define $\hat{P}_{0}=\mathbf{q}, \hat{P}_{1}=(m+1, n), \hat{P}_{2}=(m, n+1)$, and $\hat{P}_{3}=(m+1, n+1)$. Note $S$ is either triangle $\hat{P}_{0} \hat{P}_{1} \hat{P}_{2}$ or triangle $\hat{P}_{3} \hat{P}_{2} \hat{P}_{1}$. We use $\left|P_{i}-P_{j}\right|$ to denote the distance between points $P_{i}$ and $P_{j}$. Observed that

$$
\operatorname{dist}\left(S, S_{i^{\prime} j^{\prime}}\right) \leq \min \left\{\left|P_{1}-\hat{P}_{1}\right|,\left|P_{2}-\hat{P}_{1}\right|,\left|P_{1}-\hat{P}_{2}\right|,\left|P_{2}-\hat{P}_{2}\right|\right\}
$$

Now, $\left|\mathbf{q}-\mathbf{q}^{\prime}\right|=\sqrt{m^{2}+n^{2}}$,

$\left|P_{1}-\hat{P}_{1}\right|=\sqrt{(m)^{2}+(n)^{2}}=\left|P_{2}-\hat{P}_{2}\right|$,

$\left|P_{1}-\hat{P}_{2}\right|=\sqrt{(m-1)^{2}+(n+1)^{2}} ; \quad\left|P_{2}-\hat{P}_{1}\right|=\sqrt{(m+1)^{2}+(n-1)^{2}}$.

Combining with (4.82), the first inequality follows. We now turn to prove the second inequality. If $\hat{S}_{i^{\prime} j^{\prime}}$ and $S$ are not in the same row or column (see Figure 4.16), we have

$$
\operatorname{dist}\left(S_{i^{\prime} j^{\prime}}, S\right) \geq \sqrt{(m-1)^{2}+(n-1)^{2}} \geq \sqrt{m^{2}+n^{2}}-\sqrt{2}
$$

On the other hand, when $\hat{S}_{i^{\prime} j^{\prime}}$ and $S$ are in the same row or column, by the triangle inequality, we have

$$
\operatorname{dist}\left(\hat{S}_{i^{\prime} j^{\prime}}, S\right)+1 \geq\left|\mathbf{q}-\mathbf{q}^{\prime}\right|
$$

i.e.,

$$
\operatorname{dist}\left(\hat{S}_{i^{\prime} j^{\prime}}, S\right) \geq\left|\mathbf{q}-\mathbf{q}^{\prime}\right|-1 \geq\left|\mathbf{q}-\mathbf{q}^{\prime}\right|-\sqrt{2}
$$

This completes the proof.

Lemma 4.18 Assume that $K_{i^{\prime} j^{\prime}, i j}$ is an entry of $\mathbf{K}_{\mathbf{q}^{\prime} \mathbf{q}}^{i^{\prime} i}$. Then

$$
\frac{\left|\mathbf{q}-\mathbf{q}^{\prime}\right|-\sqrt{2}}{\mu^{\left(i_{0}-1\right) / 2}} \leq \operatorname{dist}\left(\hat{S}_{i^{\prime} j^{\prime}}, S_{i j}\right)<\frac{\left|\mathbf{q}-\mathbf{q}^{\prime}\right|+\sqrt{2}}{\mu^{\left(i_{0}-1\right) / 2}}
$$

Proof: We present a proof for the case $i \geq i^{\prime}$. The proof for the case $i<i^{\prime}$ is similar. First note that

$$
S_{i^{\prime} j^{\prime}}=\phi_{\mathbf{e}^{\prime}}(\Omega) \quad \text { and } \quad S_{i j}=\phi_{\mathbf{e}}(\Omega)
$$

by (4.75) we have

$$
\mathbf{q}=\gamma\left(\mathbf{e}_{c}\right), \quad \text { with } \mathbf{e}_{c}=\left(e_{0}, \ldots, e_{i^{\prime}-2}\right)
$$




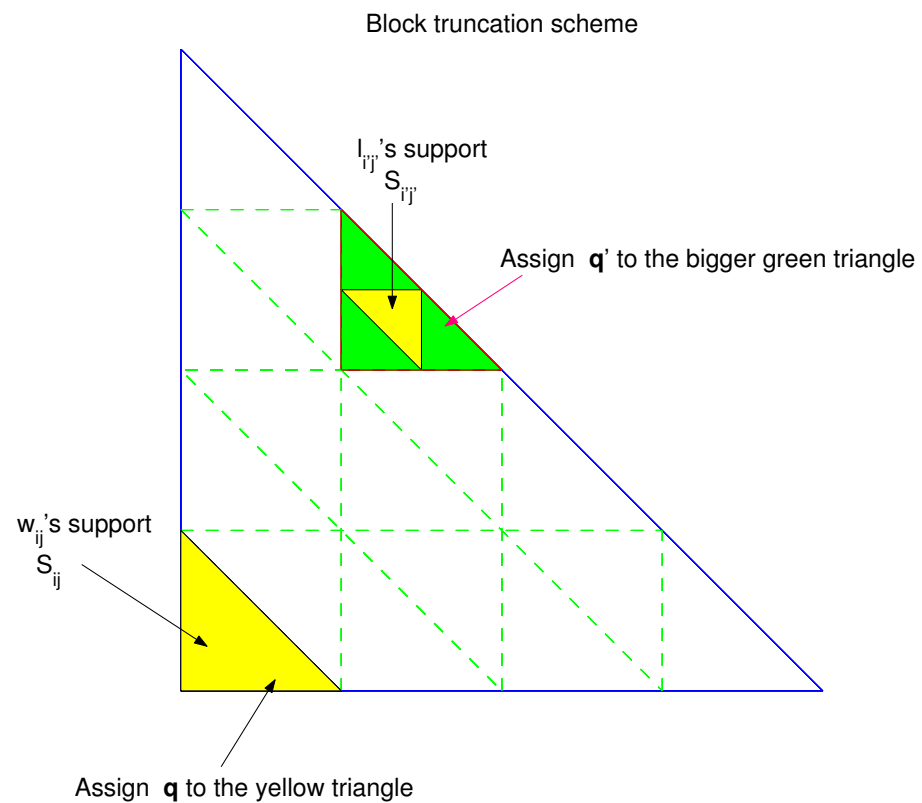

Figure 4.17: Block truncation scheme

Observe that

$$
S_{i j} \subset S:=\phi_{\mathbf{e}_{c}}(\Omega)
$$

For $\mathbf{q} \neq \mathbf{q}^{\prime}$, by Lemma 4.17

$$
\frac{\left|\mathbf{q}-\mathbf{q}^{\prime}\right|}{\mu^{\left(i^{\prime}-1\right) / 2}} \geq \operatorname{dist}\left(\hat{S}_{i^{\prime} j^{\prime}}, S\right) \geq \frac{\left|\mathbf{q}-\mathbf{q}^{\prime}\right|-\sqrt{2}}{\mu^{\left(i^{\prime}-1\right) / 2}}
$$

This yields

$$
\operatorname{dist}\left(\hat{S}_{i^{\prime} j^{\prime}}, S\right) \leq \operatorname{dist}\left(\hat{S}_{i^{\prime} j^{\prime}}, S_{i j}\right)<\operatorname{dist}\left(\hat{S}_{i^{\prime} j^{\prime}}, S\right)+\frac{\sqrt{2}}{\mu^{\left(i^{\prime}-1\right) / 2}}
$$

We now are ready to present the block truncation scheme. Define $\mathbf{p}:=\left[p_{i^{\prime} i}: p_{i^{\prime} i}>0, i^{\prime}, i \in\right.$ $\left.\mathbb{Z}_{n+1}\right]$ and let

$$
\tilde{\mathbf{K}}_{i^{\prime} i}=\left[\mathbf{K}_{\mathbf{q}^{\prime} \mathbf{q}}^{i^{\prime}}(\mathbf{p}): \mathbf{q}^{\prime}, \mathbf{q} \in \mathbb{Z}_{\mu^{\left(i_{0}-1\right) / 2}}^{2}\right]
$$

with

$$
\mathbf{K}_{\mathbf{q}^{\prime} \mathbf{q}}^{i^{\prime} i}(\mathbf{p})= \begin{cases}\mathbf{K}_{\mathbf{q}^{\prime} \mathbf{q}}^{i^{\prime} i}, & \left|\mathbf{q}-\mathbf{q}^{\prime}\right| \leq p_{i^{\prime} i} \\ 0, & \text { otherwise }\end{cases}
$$


Next theorem shows that solutions of (4.68) by this block truncation scheme has the same order of convergence and computational complexity as those by adopting the theoretical truncation scheme (4.64), provided that the truncation parameters $\mathbf{p}$ is properly chosen.

Theorem 4.19 Assume that $u \in W^{k, \infty}(\Omega)$. For some $a>0$ and $\nu^{\prime}>0$, let

$$
p_{i^{\prime} i}:=\max \left\{a \mu^{\left[b^{\prime}\left(n-i^{\prime}\right)+\left(i^{\prime}-i-1\right)\right] / 2}, \nu^{\prime}\left(\mu^{\left(-i+i^{\prime}\right) / 2}+1\right)\right\}-\sqrt{2}, \quad \text { for } i^{\prime}<i
$$

of

$$
p_{i^{\prime} i}:=\max \left\{a \mu^{\left[b^{\prime}\left(n-i^{\prime}\right)-1\right] / 2}, \nu^{\prime}\left(\mu^{\left(i-i^{\prime}\right) / 2}+1\right)\right\}-\sqrt{2}, \quad \text { for } i^{\prime} \geq i .
$$

Assume scheme (4.89), then there exists a positive constant c such that

$$
\left\|u-\tilde{u}_{n}\right\|_{\infty} \leq c f(n)^{-k / 2} \log f(n)\|u\|_{k, \infty}
$$

and

$$
\mathcal{N}\left(\tilde{\mathbf{A}}_{n}\right)=\mathcal{O}(f(n) \log f(n))
$$

where $\mathcal{N}\left(\tilde{\mathbf{A}}_{n}\right)$ denotes the number of nonzero entries in matrix $\tilde{\mathbf{A}}_{n}$.

Proof: As proved in [CMX3] if the truncation parameter $\epsilon_{i^{\prime} i}$ is chosen by (4.64), and scheme (4.63) is applied, then estimate (4.93) holds. It suffices to show that if the block truncation scheme (4.89) is assumed, scheme (4.63) is preserved. Namely, we need to show for appropriate $\mathbf{p}$, if $\left|\mathbf{q}-\mathbf{q}^{\prime}\right| \leq p_{i^{\prime} i}$, then

$$
\operatorname{dist}\left(\hat{S}_{i^{\prime} j^{\prime}}, S_{i j}\right) \leq \epsilon_{i^{\prime} i}
$$

From (4.78) we have for each pair $\left(i^{\prime}, j^{\prime}\right),(i, j) \in \mathbb{U}_{n}$, there exists $\mathbf{q}^{\prime}, \mathbf{q} \in \mathbb{Z}_{\mu^{\left(i_{0}-1\right) / 2}}^{2}$ such that $K_{i^{\prime} j^{\prime}, i j}$ is an entry of the block $\mathbf{K}_{\mathbf{q q}^{\prime}}^{i^{\prime}}$. If

$$
\left|\mathbf{q}-\mathbf{q}^{\prime}\right|<p_{i^{\prime} i}
$$

in view of (4.91), (4.92) and (4.64), we have

$$
\frac{\left|\mathbf{q}-\mathbf{q}^{\prime}\right|+\sqrt{2}}{\mu^{\left(i_{0}-1\right) / 2}} \leq \epsilon_{i^{\prime} i} .
$$


It follows from Lemma 4.18 that

$$
\operatorname{dist}\left(\hat{S}_{i^{\prime} j^{\prime}}, S_{i j}\right) \leq \epsilon_{i^{\prime} i} .
$$

According to the block truncation strategy (4.89), we have that

$$
\tilde{\mathbf{K}}^{i^{\prime} i}=\left[\mathbf{K}_{\mathbf{q}^{\prime} \mathbf{q}}^{i^{\prime} i}(\mathbf{p}):\left|\mathbf{q}-\mathbf{q}^{\prime}\right| \leq p_{i^{\prime} i}\right]
$$

which guarantees the scheme (4.63) and hence (4.93) follows. It remains to prove (4.94). To this end we set

$$
\begin{gathered}
\Lambda_{1}:=\left\{\left(i^{\prime}, j^{\prime}\right),(i, j) \in \mathbb{U}_{n}:\left|\mathbf{q}^{\prime}-\mathbf{q}\right| \leq p_{i^{\prime} i}\right\}, \\
\Lambda_{2}:=\left\{\left(i^{\prime}, j^{\prime}\right),(i, j) \in \mathbb{U}_{n}: \operatorname{dist}\left(\hat{S}_{i^{\prime} j^{\prime}}, S_{i j}\right) \leq \epsilon_{i^{\prime} i}\right\}, \\
\Lambda_{3}:=\left\{\left(i^{\prime}, j^{\prime}\right),(i, j) \in \mathbb{U}_{n}: \epsilon_{i^{\prime} i}<\operatorname{dist}\left(\hat{S}_{i^{\prime} j^{\prime}}, S_{i j}\right) \text { and }\left|\mathbf{q}^{\prime}-\mathbf{q}\right| \leq p_{i^{\prime} i}\right\} .
\end{gathered}
$$

We estimate the cardinality $\operatorname{card}\left(\Omega_{1}\right)$. we have seen inequality $\left|\mathbf{q}^{\prime}-\mathbf{q}\right| \leq p_{i^{\prime} i}$ implies that $\operatorname{dist}\left(S_{i^{\prime} j^{\prime}}, S_{i j}\right) \leq \epsilon_{i^{\prime} i}$. Thus, we have that

$$
\operatorname{card}\left(\Lambda_{1}\right)=\operatorname{card}\left(\Lambda_{2}\right)+\operatorname{card}\left(\Lambda_{3}\right)
$$

It is proved in [CMX3] that

$$
\operatorname{card}\left(\Lambda_{2}\right)=\mathcal{O}(f(n) \log f(n))
$$

Now for any fixed $i^{\prime}, i \in \mathbb{Z}_{n+1}$, there are at most $c \mu^{i_{0}-1}$ sub-blocks $\mathbf{K}_{\mathbf{q q}^{\prime}}^{i^{\prime} i}$ in $\mathbf{K}_{i^{\prime} i}$ for some positive constant $c$, satisfying that both $\epsilon_{i^{\prime} i}<\operatorname{dist}\left(\hat{S}_{i^{\prime} j^{\prime}}, S_{i j}\right)$ and $\left|\mathbf{q}^{\prime}-\mathbf{q}\right| \leq p_{i^{\prime} i}$. While each sub-block $\mathbf{K}_{\mathbf{q}^{\prime} \mathbf{q}}^{i^{\prime}}$ has at most $2 r^{2} \mu^{\left|i-i^{\prime}\right|}$ entries. Therefore

$$
\operatorname{card}\left(\Lambda_{3}\right) \leq 4 c r^{2} \sum_{i \in \mathbb{Z}_{n+1}} \sum_{i^{\prime} \in \mathbb{Z}_{i+1}} \mu^{\left|i-i^{\prime}\right|+i_{0}-1}=\mathcal{O}(f(n) \log f(n)) .
$$

This with (4.101) establishes (4.94).

For any given triangle $\hat{S}_{i^{\prime} j^{\prime}}$ associated with index $\mathbf{q}^{\prime}$, the block truncation scheme is expected to catch any triangle $S$ associated with index q surrounding $\hat{S}_{i^{\prime} j^{\prime}}$. On the other hand, we don't want too many triangles are caught by the scheme. A simple analysis shows that for triangles which are surrounding $\hat{S}_{i^{\prime} j^{\prime}},\left|\mathbf{q}^{\prime}-\mathbf{q}\right| \leq \sqrt{2}$, hence $\sqrt{2}<p_{i^{\prime} i} \leq \sqrt{3}, i^{\prime}, i \in \mathbb{Z}_{n+1}$ 
would be a very good choice. Following examples demonstrates this.

For the compression rates of the block truncation scheme with $p_{i^{\prime} i}=\sqrt{3}$ please see Table 4.4. For comparing compression rates with those of other schemes, please see Figure 4.8. Note the block truncation scheme is not contained in any of the schemes discussed in the previous section. While for distance computing scheme: strategy 1 contains strategy 2 and in turn strategy 2 contains strategy 3 . When level $n<5$, the block truncation scheme has maximum compression rate, yet, the convergence rate is very satisfactory. When $n>5$, the compression rate of block truncation scheme is between that of strategy 2 and that of strategy 3, nevertheless, block truncation scheme remains to give good solution. This suggests that, even compared to strategy 2, block truncation scheme can give more or less the same good solution but with less computational effort. In view of Figure 4.9 and the computing results in Table 6.1 and Table 6.2 in solving the integral equations (6.1), when $n=8$, the block truncation scheme can give amazing satisfactory results. Therefore, we conclude, the block truncation scheme is the best one among all the practical compression strategies we discussed so far.

Example 4.20 In this example, we solve the integral equation (6.1). To test if the proposed block truncation strategy is good enough, all the elements are computed by analytical method and the linear system of equations are solved by standard Gaussian elimination method. The results are tabulated in Table 4.11. We can see that the compressed solutions by block truncation scheme follow very closely the uncompressed solution. The block truncation parameter $p_{i^{\prime} i}$ is set as $\sqrt{3}$. The convergent rates for different levels are plotted in Figure 4.9.

\subsection{Quadrature Error Control}

After discretizing the integral equation (2.2) with compression applied, we obtain a linear system

$$
\mathbf{E}_{n}-\tilde{\mathbf{K}}_{n}=\mathbf{f}_{n} .
$$

Since the nonzero entries of the coefficient matrix $\tilde{\mathbf{K}}_{n}$ (obtained after compression) are usually obtained by quadrature, we actually solve a perturbed version of the linear system (4.102), 
Block truncation scheme with $p_{i^{\prime} i}=\sqrt{3}$

\begin{tabular}{|c|c|c|c|c|c|}
\hline & \multicolumn{2}{|c|}{ full collocation solution $u_{n}$} & \multicolumn{3}{|c|}{ compressed solution $\tilde{u}_{n}$} \\
$n$ & $L^{\infty}$-error & order & $L^{\infty}$-error & order & compression rate \\
\hline 1 & $1.513187 \mathrm{e}-1$ & & $1.513187 \mathrm{e}-1$ & & 1.000000 \\
2 & $3.599605 \mathrm{e}-2$ & 2.0717 & $3.599605 \mathrm{e}-2$ & 2.0717 & 1.000000 \\
3 & $7.499082 \mathrm{e}-3$ & 2.2631 & $7.493692 \mathrm{e}-3$ & 2.2641 & 0.758301 \\
4 & $1.849162 \mathrm{e}-3$ & 2.0198 & $1.851165 \mathrm{e}-3$ & 2.0172 & 0.416626 \\
5 & $4.650610 \mathrm{e}-4$ & 1.9914 & $4.683404 \mathrm{e}-4$ & 1.9828 & 0.181021 \\
\hline
\end{tabular}

Table 4.11: Comparison of solutions obtained by the full collocation method and by the compressed method (with block truncation scheme).

say,

$$
\left(\mathbf{E}_{\mathbf{n}}-\tilde{\tilde{\mathbf{K}}}_{n}\right) \tilde{\tilde{\mathbf{u}}}_{n}=\mathbf{f}_{n}
$$

where

$$
\tilde{\tilde{\mathbf{K}}}_{n}=\left(\tilde{\tilde{K}}_{i^{\prime} j^{\prime}, i j}\right) \text { with } \quad \tilde{\tilde{K}}_{i^{\prime} j^{\prime}, i j}=\tilde{K}_{i^{\prime} j^{\prime}, i j}+\delta_{i^{\prime} j^{\prime}, i j}
$$

and $\delta_{i^{\prime} j^{\prime}, i j}$ denotes the quadrature error for computing the integral $K_{i^{\prime} j^{\prime}, i j}$ (or equivalently, $\left.\tilde{K}_{i^{\prime} j^{\prime}, i j}\right)$. We wish to control quadrature errors, such that they will not affect the convergent rate of the approximation solutions. In the following we establish a rule for the quadrature accuracy so that the solution $\tilde{\tilde{u}}_{n}$ obtained still satisfies the same order of error estimate as stated in Theorem 4.14. Let $\delta_{i^{\prime} i}$ be the quadrature error for the block matrix $\tilde{\mathbf{K}}_{i^{\prime} i}$, namely

$$
\delta_{i^{\prime} i}=\max _{j^{\prime}, j}\left|K_{i^{\prime} j^{\prime}, i j}-\tilde{\tilde{K}}_{i^{\prime} j^{\prime}, i j}\right|, \quad j \in \mathbb{Z}_{w(i)}, \quad j^{\prime} \in \mathbb{Z}_{w\left(i^{\prime}\right)} .
$$

Lemma 4.21 Suppose that for a given constant $\nu>1$ and $0<\sigma^{\prime}<2-\sigma$, the quadrature rule have an accuracy such that

$$
\delta_{i^{\prime} i} \leq c \epsilon_{i^{\prime} i}^{-\eta} d_{i}^{2+k} d_{i^{\prime}}^{k}, \quad i^{\prime}, i \in \mathbb{Z}_{n+1},
$$

for some constant $c$. Then there exist a constant $c_{1}$ whenever $\epsilon_{i^{\prime} i} \geq r\left(d_{i}+d_{i^{\prime}}\right)$ then

$$
\left\|\mathbf{K}_{i^{\prime} i}-\tilde{\tilde{\mathbf{K}}}_{i^{\prime} i}\right\|_{\infty} \leq c_{1} \epsilon_{i^{\prime} i}^{-\eta}\left(d_{i} d_{i^{\prime}}\right)^{k}, \quad i^{\prime}, i \in \mathbb{Z}_{n+1},
$$

where $\epsilon_{i^{\prime} i}$ is the truncation parameter for the block matrix $\mathbf{K}_{i^{\prime} i}$ and $\eta:=2 k-\sigma^{\prime}$. 
Yi Wang

Proof: We first note

$$
\left\|\mathbf{K}_{i^{\prime} i}-\tilde{\tilde{\mathbf{K}}}_{i^{\prime} i}\right\|_{\infty} \leq\left\|\mathbf{K}_{i^{\prime} i}-\tilde{\mathbf{K}}_{i^{\prime} i}\right\|_{\infty}+\left\|\tilde{\mathbf{K}}_{i^{\prime} i}-\tilde{\tilde{\mathbf{K}}}_{i^{\prime} i}\right\|_{\infty} .
$$

From Lemma 3.2 of [CMX3] we have

$$
\left\|\mathbf{K}_{i^{\prime} i}-\tilde{\mathbf{K}}_{i^{\prime} i}\right\|_{\infty} \leq c \epsilon_{i^{\prime} i}^{-\eta}\left(d_{i^{\prime}} d_{i}\right)^{k} \quad i, i^{\prime} \in \mathbb{Z}_{n+1}
$$

It then follows from (4.104) that

$$
\begin{aligned}
\left\|\tilde{\mathbf{K}}_{i^{\prime} i}-\tilde{\tilde{\mathbf{K}}}_{i^{\prime} i}\right\|_{\infty} & =\max _{j^{\prime} \in \mathbb{Z}_{w\left(i^{\prime}\right)}} \sum_{j \in \mathbb{Z}_{w(i)} \backslash \mathbb{Z}_{i^{\prime} j^{\prime}}(\epsilon)}\left|K_{i^{\prime} j^{\prime}, i j}-\tilde{\tilde{K}}_{i^{\prime} j^{\prime}, i j}\right| \\
& \leq \max _{j^{\prime} \in \mathbb{Z}_{w\left(i^{\prime}\right)}} \sum_{j \in \mathbb{Z}_{w(i)}}\left|K_{i^{\prime} j^{\prime}, i j}-\tilde{\tilde{K}}_{i^{\prime} j^{\prime}, i j}\right| \leq c w(i) \epsilon_{i^{\prime} i}^{-\eta}\left(d_{i} d_{i^{\prime}}\right)^{k} d_{i}^{2} \\
& \leq c \epsilon_{i^{\prime} i}^{-\eta}\left(d_{i} d_{i^{\prime}}\right)^{k},
\end{aligned}
$$

where

$$
\mathbb{Z}_{i^{\prime} j^{\prime}}(\epsilon):=\left\{j: j \in \mathbb{Z}_{w(i)}, \operatorname{dist}\left(S_{i j}, \hat{S}_{i^{\prime} j^{\prime}}\right)>\epsilon_{i^{\prime} i}\right\} .
$$

The last inequality is obtained by using

$$
w(i) \leq c \mu^{i}
$$

and

$$
d_{i}=c \mu^{-i / 2} .
$$

This completes the proof.

Corrolary 4.22 If the truncation parameter $\epsilon_{i^{\prime} i}$ is chosen to be $c\left(d_{i}+d_{i^{\prime}}\right)$ for some constant c, then (4.105) is satisfied by choosing

$$
\delta_{i^{\prime} i} \leq c\left(d_{i}\right)^{k+2} d_{i^{\prime}}^{k}
$$

or equivalently by choosing

$$
\delta_{i^{\prime} i} \leq c \mu^{-k\left(\frac{i}{2}+\frac{i^{\prime}}{2}\right)-i}
$$

Proof: From (4.107) and $d_{i} \leq c, i \in \mathbb{N}_{0}$, we obtain

$$
c 2^{-\eta} \leq\left(d_{i}+d_{i^{\prime}}\right)^{-\eta} .
$$


Yi Wang

Now

$$
c\left(d_{i} d_{i^{\prime}}\right)^{k} d_{i}^{2}=c^{\prime} 2^{-\eta}\left(d_{i} d_{i^{\prime}}\right)^{k} d_{i}^{2} \leq c\left(d_{i}+d_{i^{\prime}}\right)^{-\eta}\left(d_{i} d_{i^{\prime}}\right)^{k} d_{i}^{2}=c \epsilon_{i^{\prime} i}^{-\eta}\left(d_{i} d_{i^{\prime}}\right)^{k} d_{i}^{2}
$$

Thus (4.104) is satisfied, and (4.105) follows. (4.109) follows by directly using (4.108) and (4.107).

Let $m_{i^{\prime} i}$ be the number used in the quadrature rule (see (4.36) ) to compute entries of the block matrix $\tilde{\tilde{\mathbf{K}}}_{i^{\prime} i}$. Assume that $m_{00}$ is chosen. If the quadrature rule (4.36) has error estimate $O\left(m^{-2 k}\right)$, then $m_{i^{\prime} i}$ can be chosen as

$$
m_{i^{\prime} i}=c m_{00}\left(\epsilon_{i^{\prime} i}\right)^{\eta / 2 k}\left(d_{i} d_{i^{\prime}}\right)^{-1 / 2} d_{i}^{-\frac{1}{k}} .
$$

In particular, if $\epsilon_{i^{\prime} i}=c\left(d_{i}+d_{i^{\prime}}\right)$, we can choose

$$
m_{i^{\prime} i}=c m_{00}\left(d_{i} d_{i^{\prime}}\right)^{-1 / 2}\left(d_{i}\right)^{-1 / k} .
$$

Theorem 4.23 Assume that the quadrature rule used to compute entries of $\tilde{\tilde{\mathbf{K}}}_{\mathbf{n}}$ has error estimate $O\left(m^{-2 k}\right)$. Choose $m_{00}$ large enough such that (4.104) is satisfied for $i^{\prime}=i=0$ and identify other $m_{i^{\prime} i}$ by (4.110) (in the case of $\epsilon_{i^{\prime} i}=c\left(d_{i^{\prime}}+d_{i}\right)$, (4.111) is used), and the truncation parameter $\epsilon_{i^{\prime} i}, i^{\prime}, i \in \mathbb{Z}_{n+1}$ are chosen according to (4.64). Then there exists a positive constant $c$ and a positive integer $N$ such that for all $n>N$,

$$
\left\|u-\tilde{\tilde{u}}_{n}\right\|_{\infty} \leq c f(n)^{-k / 2} \log f(n)\|u\|_{\infty},
$$

where, $\tilde{\tilde{u}}_{n}=\sum_{(i, j) \in \mathbb{U}_{n}} \tilde{\tilde{u}}_{i j} w_{i j}, \quad$ with $\quad \tilde{\tilde{\mathbf{u}}}_{n}=\left[\tilde{\tilde{u}}_{i j},(i, j) \in \mathbb{U}_{n}\right]$.

Proof: Since the quadrature rule has error estimate $O\left(m^{-2 k}\right)$, if $m_{00}$ is large enough to meet (4.104), there holds

$$
c m_{00}^{-2 k} \leq \epsilon_{00}^{-\eta} d_{0}^{2 k+2}
$$

By (4.110) we have

$$
m_{i^{\prime} i}^{-2 k}=c\left(m_{00}\right)^{-2 k}\left(\epsilon_{i^{\prime} i}^{\eta / 2 k}\left(d_{i} d_{i^{\prime}}\right)^{-1 / 2} d_{i}^{-1 / k}\right)^{-2 k} \leq c \epsilon_{i^{\prime} i}^{-\eta}\left(d_{i} d_{i^{\prime}}\right)^{k} d_{i}^{2} .
$$

Therefore (4.104) is satisfied. For the case when $\epsilon_{i^{\prime} i}=c\left(d_{i}+d_{i^{\prime}}\right)$, use (4.111), (4.104) is satisfied. The remaining proof can follow that of [CMX3](see [CMX3] Lemma 4.1, Lemma 4.2 and Theorem 4.4). 
We next discuss the quadrature error control from another perspective. Assuming both the coefficient matrix $\tilde{\mathbf{K}}_{n}$ and $\mathbf{f}_{n}$ are obtained by numerical quadrature. In this case, we solve a perturbed version of the linear system (4.102),

$$
\left(\mathbf{E}_{n}-\tilde{\tilde{\mathbf{K}}}_{n}\right) \tilde{\tilde{\mathbf{u}}}_{n}=\tilde{\tilde{\mathbf{f}}}_{n}
$$

where

$$
\begin{aligned}
& \tilde{\tilde{\mathbf{K}}}_{n}=\left(\tilde{\tilde{K}}_{i^{\prime} j^{\prime}, i j}\right) \text { with } \quad \tilde{\tilde{K}}_{i^{\prime} j^{\prime}, i j}=\tilde{K}_{i^{\prime} j^{\prime}, i j}+\delta_{i^{\prime} j^{\prime}, i j} \text {, } \\
& \tilde{\tilde{\mathbf{f}}}=\left(\tilde{\tilde{f}}_{i^{\prime} j^{\prime}}\right) \quad \text { with } \quad \tilde{\tilde{f}}_{i^{\prime} j^{\prime}}=f_{i^{\prime} j^{\prime}}+\delta_{i^{\prime} j^{\prime}} \text {. }
\end{aligned}
$$

$\delta_{i^{\prime} j^{\prime}, i j}$ and $\delta_{i^{\prime} j^{\prime}}$ denote the quadrature error for computing the double integral $K_{i^{\prime} j^{\prime}, i j}$ and single integral $f_{i^{\prime} j^{\prime}}$, respectively. We desire the solution $\tilde{\tilde{u}}_{n}$ still satisfies the same order of error estimate as the compressed collocation method (see Theorem 4.14). Namely,

$$
\left\|\tilde{u}_{n}-u\right\|_{\infty}=O\left(n \mu^{-k n / 2}\right)
$$

where, $k$ is the total degree of piecewise polynomials in $\mathbb{F}_{n}$. Since $w_{i j},(i, j) \in \mathbb{U}$ is uniformly bounded (Lemma 3.4), we have that

$$
\left\|\tilde{u}_{n}-\tilde{\tilde{u}}_{n}\right\|_{\infty} \leq C\left\|\tilde{\mathbf{u}}_{n}-\tilde{\tilde{\mathbf{u}}}_{n}\right\|_{\infty} .
$$

Moreover, because $\tilde{\mathbf{u}}_{n}-\tilde{\tilde{\mathbf{u}}}_{n}$ solves the linear system

$$
\left(\mathbf{E}_{n}-\tilde{\mathbf{K}}_{n}\right)\left(\tilde{\mathbf{u}}_{n}-\tilde{\tilde{\mathbf{u}}}_{n}\right)=\mathbf{f}_{n}-\tilde{\tilde{\mathbf{f}}}_{n}+\left(\tilde{\mathbf{K}}_{n}-\tilde{\tilde{\mathbf{K}}}_{n}\right) \tilde{\tilde{\mathbf{u}}}_{n}
$$

we conclude that

$$
\left\|\tilde{u}_{n}-\tilde{\tilde{u}}_{n}\right\|_{\infty} \leq C\left\|\tilde{\mathbf{u}}_{n}-\tilde{\tilde{\mathbf{u}}}_{n}\right\|_{\infty}=\mathrm{O}\left(\left\|\mathbf{f}_{n}-\tilde{\tilde{\mathbf{f}}}_{n}\right\|_{\infty}\right)+\mathrm{O}\left(\left\|\tilde{\mathbf{K}}_{n}-\tilde{\tilde{\mathbf{K}}}_{n}\right\|_{\infty}\right) .
$$

Now,

$$
\left\|\mathbf{f}_{n}-\tilde{\tilde{\mathbf{f}}}_{n}\right\|_{\infty}=\max _{\left(i^{\prime}, j^{\prime}\right) \in \mathbb{U}_{n}}\left|\delta_{i^{\prime} j^{\prime}}\right|
$$

and

$$
\left\|\tilde{\mathbf{K}}_{n}-\tilde{\tilde{\mathbf{K}}}_{n}\right\|_{\infty} \leq \max _{\left(i^{\prime}, j^{\prime}\right) \in \mathbb{U}_{n}} \sum_{(i, j) \in \mathbb{U}_{n}}\left|\delta_{i^{\prime} j^{\prime}, i j}\right| \leq c \mu^{n} \max _{\left(i^{\prime}, j^{\prime}\right),(i, j) \in \mathbb{U}_{n}}\left|\delta_{i^{\prime} j^{\prime}, i j}\right| .
$$

Thus, by letting

$$
\delta^{(n)}:=\max _{\left(i^{\prime}, j^{\prime}\right),(i, j) \in \mathbb{U}_{n}}\left\{\left|\delta_{i^{\prime} j^{\prime}}\right|,\left|\delta_{i^{\prime} j^{\prime}, i j}\right|\right\}
$$


we have

$$
\left\|\tilde{u}_{n}-\tilde{\tilde{u}}_{n}\right\|_{\infty}=\mathrm{O}\left(\mu^{n} \delta^{(n)}\right) .
$$

In order for $\tilde{\tilde{u}}_{n}$ to be an approximation to $u_{n}$ in the same order as $\tilde{u}_{n}$ to $u_{n}$ in (4.113), we need to require that the quadrature error $\delta^{(n)}$ be reduced in the order of

$$
\delta^{(n)} \sim n \mu^{\frac{-k n}{2}-n}
$$

as $n$ increases. When apply the quadrature rule presented in Section 4.3, this requirement implies that the number of subdivisions, $m_{n}$, according to $n$ need to be increased as

$$
m_{n} \sim n^{-\frac{1}{2 k}} \mu^{\frac{(k+2) n}{4 k}}
$$

That is,

$$
\frac{m_{n+1}}{m_{n}}=\left(\frac{n}{n+1}\right)^{\frac{1}{2 k}} \mu^{\frac{(k+2)}{4 k}},
$$

which implies that, as the level of resolution increases by 1 , the number of subdivisions in the quadrature rule should be increased by approximately $\mu^{\frac{k+2}{4 k}}$ times, in order to maintain the error order of the approximation solution of the collocation method as shown in (4.113). In our examples, $k=2, \mu=4$, hence this factor is approximately $4^{1 / 2}=2.0$. This method to control the quadrature error is demonstrated in Example 4.25. When the level $i \geq 2$, the domain of integration is no longer the whole domain and getting smaller and smaller. If the element $f_{i^{\prime} j^{\prime}}$ is obtained also by numerical quadrature, then its domain of integration is always the whole original domain. In view of Table 4.1 and Table 4.2, in practical computing, we can expect to use less number of subdivisions to compute elements $K_{i^{\prime} j^{\prime}, i j}$, for $i \geq 2$ to obtain the same order of convergence rate. This idea is demonstrated in Example 4.26.

We summarize above discussion in the following theorem:

Theorem 4.24 If the number of subdivisions $m_{n}$ used in the adaptive quadrature rule (4.36) is chosen as (4.114) for solving the perturbed equation (4.112), then

$$
\left\|u-\tilde{\tilde{u}}_{n}\right\|_{\infty} \leq c f(n)^{-k / 2} \log f(n)\|u\|_{\infty} .
$$

Example 4.25 In this example, we test different number of subdivisions in the quadrature rule for different level $n$ when solving the integral equation (6.1). But for fixed $n$, the computing of all entries assumes the same number of subdivisions $m_{n}$ for the quadrature rule. 


\begin{tabular}{|c|c|c|c|c|c|}
\hline & \multicolumn{2}{|c|}{ without quadrature } & \multicolumn{3}{|c|}{ with quadrature } \\
$n$ & $L^{\infty}$-error & order & $m_{n}$ & $L^{\infty}$-error & order \\
\hline 1 & $1.513187 \mathrm{e}-1$ & & 10 & $1.512309 \mathrm{e}-1$ & \\
2 & $3.599605 \mathrm{e}-2$ & 2.0717 & 10 & $3.540331 \mathrm{e}-2$ & 2.0948 \\
3 & $7.499082 \mathrm{e}-3$ & 2.2631 & 10 & $7.526215 \mathrm{e}-3$ & 2.2339 \\
4 & $1.849162 \mathrm{e}-3$ & 2.0198 & 20 & $1.864510 \mathrm{e}-3$ & 2.0131 \\
5 & $4.650610 \mathrm{e}-4$ & 1.9914 & 40 & $4.657507 \mathrm{e}-4$ & 2.0012 \\
\hline
\end{tabular}

Table 4.12: Comparison of uncompressed solutions, with and without numerical quadratures, using different $m_{n}$ for different $n$.

To eliminate other affecting factors, the linear system of equations are solved by standard Gaussian elimination method. No compression is assumed. The results are tabulated in Table 4.12. We can see from Table 4.12 that the results by using the quadrature control rule (4.115) are very close to those obtained without quadrature rule.

Example 4.26 In this example, we test for fixed $n$, different number of subdivisions for different levels used in the quadrature rule when solving the integral equation (6.1). The linear system of equations are solved by standard Gaussian elimination method. No compression is assumed. The results are tabulated in Table 4.13. In Table 4.13, $\left(m_{1}, m_{2}\right)$ means that when the level $i, i \in \mathbb{Z}_{n+1}$ corresponding to wavelet functions $w_{i j}$ less than 2 , the number of subdivisions used in quadrature is $m_{1}$, and when $i \geq 2$, the number of subdivisions used in quadrature assumes $m_{2}$. While for computing $f_{i^{\prime} j^{\prime}}$, the number of subdivisions used is always $m_{1}$. We can see from results in Table 4.13 that the this quadrature control method also works very well too. But, the computational cost compared to that of Example 4.25 for quadrature has been dramatically saved.

\subsection{Iteration Methods}

In this section, we develop multilevel algorithms to solve the resulting linear system by making use of multilevel structures of the approximation spaces $\mathbb{F}_{n}$ of $\mathbb{X}$. In [CMX4] a two- 


\begin{tabular}{|c|c|c|c|c|l|}
\hline & \multicolumn{2}{|c|}{ without quadrature } & \multicolumn{3}{|c|}{ with quadrature } \\
$n$ & $L^{\infty}$-error & order & $\left(m_{1}, m_{2}\right)$ & $L^{\infty}$-error & order \\
\hline 1 & $1.513187 \mathrm{e}-1$ & & $(10,10)$ & $1.512309 \mathrm{e}-1$ & \\
2 & $3.599605 \mathrm{e}-2$ & 2.0717 & $(10,10)$ & $3.540331 \mathrm{e}-2$ & 2.0948 \\
3 & $7.499082 \mathrm{e}-3$ & 2.2631 & $(20,10)$ & $7.548485 \mathrm{e}-3$ & 2.2296 \\
4 & $1.849162 \mathrm{e}-3$ & 2.0198 & $(20,10)$ & $1.852722 \mathrm{e}-3$ & 2.0265 \\
5 & $4.650610 \mathrm{e}-4$ & 1.9914 & $(40,20)$ & $4.638874 \mathrm{e}-4$ & 1.9978 \\
\hline
\end{tabular}

Table 4.13: Comparison of uncompressed solutions, with and without numerical quadratures, using different numbers of subdivisions for different levels.

level method is introduced and analyzed for solving operator equations in a Banach Space. [CWX1] also provides a two-level method called augmentation method utilizing the multilevel structure of the approximation space. In [FMX], iterative methods are developed for solving the linear system resulting from wavelet Galerkin discretization.

Remember that the wavelet collocation scheme for equation (2.2) is to seek $u_{n} \in \mathbb{X}$ (hence $u_{n} \in \mathbb{F}_{n}$ ) satisfying

$$
\left(\mathcal{I}-\mathcal{P}_{n} \mathcal{K}\right) u_{n}=\mathcal{P}_{n} f
$$

or equivalently,

$$
\left(\mathcal{I}-\mathcal{P}_{n} \mathcal{K} \mathcal{P}_{n}\right) u_{n}=\mathcal{P}_{n} f
$$

where, $\mathcal{P}_{n}$ is defined in (3.52) and we have

$$
\mathbb{F}_{n}=\mathcal{P}_{n} \mathbb{X}
$$

It is well known (cf. [At2]) by the compactness of $\mathcal{K}$ and the pointwise convergence (see Lemma 3.7) of $\mathcal{P}_{n}$ that, for any sufficient large $n$, there exists a constant $C$, such that

$$
\left\|\left(\mathcal{I}-\mathcal{P}_{n} \mathcal{K}\right)^{-1}\right\| \leq C
$$

and

$$
\lim _{n \rightarrow \infty}\left\|\mathcal{K}-\mathcal{P}_{n} \mathcal{K}\right\|=\lim _{n \rightarrow \infty}\left\|\mathcal{K}-\mathcal{K} \mathcal{P}_{n}\right\|=0 .
$$

Recall we have required the nestedness of the approximation spaces, namely

$$
\mathbb{F}_{n} \subset \mathbb{F}_{n+1}, n \in \mathbb{N}_{0},
$$


and the space $\mathbb{F}_{n}$ is decomposed into a direct sum of a family of multiscale spaces, i.e.,

$$
\mathbb{F}_{n}=\mathbb{W}_{0} \oplus \mathbb{W}_{1} \oplus \ldots \oplus \mathbb{W}_{n}
$$

Note $\mathbb{F}_{0}=\mathbb{W}_{0}$ and

$$
\mathbb{F}_{n}=\mathbb{F}_{n-1} \oplus W_{n}, \quad n=1,2, \ldots
$$

Next lemma shows that

$$
\mathcal{Q}_{n}:=\mathcal{P}_{n}-\mathcal{P}_{n-1}, \quad n=1,2, \ldots
$$

are projections on $\mathbb{X}$.

Lemma 4.27 Let $\mathcal{P}_{1}, \mathcal{P}_{2}$ be projections on a Banach space $\mathbb{X}$, Then $\mathcal{Q}=\mathcal{P}_{2}-\mathcal{P}_{1}$ is a projection if and only if

$$
\mathbb{Y}_{1} \subset \mathbb{Y}_{2}
$$

where $\mathbb{Y}_{j}=\mathcal{P}_{j}, j=1,2$.

Proof: Assume $\mathcal{Q}=\mathcal{P}_{2}-\mathcal{P}_{1}$ is a projection, then

$$
\mathcal{P}_{2}-\mathcal{P}_{1}=\mathcal{P}_{2}+\mathcal{P}_{1}-\mathcal{P}_{2} \mathcal{P}_{1}-\mathcal{P}_{1} \mathcal{P}_{2}
$$

i.e,

$$
2 \mathcal{P}_{1}=\mathcal{P}_{2} \mathcal{P}_{1}+\mathcal{P}_{1} \mathcal{P}_{2}
$$

Multiply above formula by $\mathcal{P}_{2}$ from left and right respectively, we have

$$
\mathcal{P}_{1} \mathcal{P}_{2}=\mathcal{P}_{2} \mathcal{P}_{1}=\mathcal{P}_{1}
$$

Thus for $x \in \mathbb{X}, \mathcal{P}_{1} x=\mathcal{P}_{2} \mathcal{P}_{1} x$, namely, $\mathbb{Y}_{1} \subset \mathbb{Y}_{2}$. Conversely, let $\mathbb{Y}_{1} \subset \mathbb{Y}_{2}$, for $x \in \mathbb{X}$, $\mathcal{P}_{1} x \in \mathbb{Y}_{2}$, thus $\mathcal{P}_{2} \mathcal{P}_{1} x=\mathcal{P}_{1} x$, hence, $\mathcal{P}_{2} \mathcal{P}_{1}=\mathcal{P}_{1}$. We then have $\mathcal{P}_{1} \mathcal{P}_{2} \mathcal{P}_{1}=\mathcal{P}_{1} \mathcal{P}_{1}$, which is equivalent to $\left(\mathcal{P}_{1} \mathcal{P}_{2}-\mathcal{P}_{1}\right) \mathcal{P}_{1}=0$, leading to $\mathcal{P}_{1} \mathcal{P}_{2}=\mathcal{P}_{1}$ in any case. This completes the proof.

From Lemma 4.27, we obtain

$$
\mathbb{W}_{n}=\mathcal{Q}_{n} \mathbb{F}_{n}, \quad n=1,2, \ldots
$$


This yields, for $l, k \geq 0$,

$$
\mathcal{P}_{k+\ell}=\mathcal{P}_{k}+\mathcal{Q}_{k+1}+\cdots+\mathcal{Q}_{k+\ell}
$$

Observe that (4.122) yields, for $k \geq 0, \ell \geq 1$, and $n=k+\ell$,

$$
\mathbb{F}_{n}=\mathbb{F}_{k} \oplus \mathbb{W}_{k+1} \oplus \cdots \oplus \mathbb{W}_{n}
$$

For the convenience of description, let's identify $\left[f_{0}, g_{1}, \ldots, g_{\ell}\right]^{T}$ in $\mathbb{F}_{k} \times \mathbb{W}_{k+1} \times \cdots \times W_{k+\ell}$ with the vector

$$
f_{0}+g_{1}+\cdots+g_{\ell} \in \mathbb{F}_{k} \oplus \mathbb{W}_{k+1} \oplus \cdots \oplus \mathbb{W}_{k+\ell}
$$

In particular, for $u_{k+\ell} \in \mathbb{F}_{k+\ell}$,

$$
u_{k+\ell}=u_{k, 0}+v_{k, 1}+\cdots+v_{k, \ell}=\left[u_{k, 0}, v_{k, 1}, \cdots, v_{k, \ell}\right]^{T},
$$

where $u_{k, 0} \in \mathbb{F}_{k}$ and $v_{k, i} \in \mathbb{W}_{k+i}(1 \leq i \leq \ell)$. Now we define, for $n, m \geq 0$,

$$
\mathcal{K}_{n}=\mathcal{P}_{n} \mathcal{K P}_{n}, \quad \mathcal{K}_{n, m}=\mathcal{Q}_{n} \mathcal{K} \mathcal{Q}_{m}
$$

$$
\mathcal{B}_{n, m}=\mathcal{P}_{n} \mathcal{K} \mathcal{Q}_{m} \text { for } n<m, \quad \text { and } \quad \mathcal{C}_{n, m}=\mathcal{Q}_{n} \mathcal{K} \mathcal{P}_{m} \text { for } n>m
$$

With these notations, the operator $\mathcal{K}_{k+\ell}$ can be identified with

$$
\mathcal{A}_{k, \ell}=\left[\begin{array}{cccc}
\mathcal{K}_{k} & \mathcal{B}_{k, k+1} & \cdots & \mathcal{B}_{k, k+\ell} \\
\mathcal{C}_{k+1, k} & \mathcal{K}_{k+1, k+1} & \cdots & \mathcal{K}_{k, k+\ell} \\
\vdots & \vdots & \ddots & \vdots \\
\mathcal{C}_{k+\ell, k} & \mathcal{K}_{k+\ell, k+1} & \cdots & \mathcal{K}_{k+\ell, k+\ell}
\end{array}\right]
$$

This notation was first introduced by [CMX4]. Thus, equation (4.116) or (4.117) can be written into

$$
\mathbf{u}_{k, \ell}-\mathcal{A}_{k, \ell} \mathbf{u}_{k, \ell}=\mathbf{f}_{k, \ell}
$$

where $\mathbf{u}_{k, \ell}=\left[u_{k, 0}, v_{k, 1}, \ldots, v_{k, \ell}\right]^{T}$ and $\mathbf{f}_{k, \ell}=\left[\mathcal{P}_{k} f, \mathcal{Q}_{k+1} f, \ldots, \mathcal{Q}_{k+\ell} f\right]^{T}$. Let

$$
\mathcal{U}_{k, \ell}=\left[\begin{array}{ccccc}
O & \mathcal{B}_{k, k+1} & \mathcal{B}_{k, k+2} & \cdots & \mathcal{B}_{k, k+\ell} \\
& O & \mathcal{K}_{k+1, k+2} & \cdots & \mathcal{K}_{k+1, k+\ell} \\
& & \ddots & & \vdots \\
& & & \ddots & \mathcal{K}_{k+\ell-1, k+\ell} \\
& & & & O
\end{array}\right]
$$




$$
\mathcal{L}_{k, \ell}=\left[\begin{array}{cccc}
O & & & \\
\mathcal{C}_{k+1, k} & \mathcal{K}_{k+1, k+1} & & \\
\vdots & \vdots & \ddots & \\
\mathcal{C}_{k+\ell, k} & \mathcal{K}_{k+\ell, k+1} & \cdots & \mathcal{K}_{k+\ell, k+\ell}
\end{array}\right]
$$

and

$$
\mathcal{D}_{k, \ell}=\left[\begin{array}{cccc}
\mathcal{I}-\mathcal{K}_{k} & & & \\
& \mathcal{I} & & \\
& & \ddots & \\
& & & \mathcal{I}
\end{array}\right]
$$

where the $\mathcal{I}$ 's on the diagonal are identity operators on the corresponding subspaces $\mathbb{F}_{k}$, $\mathbb{W}_{k+1}, \ldots, \mathbb{W}_{k+\ell}$. Note

$$
\mathcal{D}_{k, \ell}=\mathcal{I}-\mathcal{A}_{k, \ell}+\mathcal{U}_{k, \ell}+\mathcal{L}_{k, \ell}
$$

and it is in fact the operator $\mathcal{I}-\mathcal{K}_{k}$ on $\mathbb{X}$. Now, equation (4.125) becomes

$$
\left(\mathcal{D}_{k, \ell}-\mathcal{U}_{k, \ell}-\mathcal{L}_{k, \ell}\right) \mathbf{u}_{k, \ell}=\mathbf{f}_{k, \ell}
$$

(4.126) suggests following two iterative schemes in terms of operator forms:

- Jacobi type iteration:

$$
\mathcal{D}_{k, \ell} \mathbf{u}_{k, \ell}^{(\mu+1)}=\left(\mathcal{U}_{k, \ell}+\mathcal{L}_{k, \ell}\right) \mathbf{u}_{k, \ell}^{(\mu)}+\mathbf{f}_{k, \ell}, \quad \mu \in \mathbb{N}_{0}
$$

with any initial approximation $\mathbf{u}_{k, \ell}^{(0)}$.

- Gauss-Seidel type iteration:

$$
\left(\mathcal{D}_{k, \ell}-\mathcal{U}_{k, \ell}\right) \mathbf{u}_{k, \ell}^{(\mu)}=\mathcal{L}_{k, \ell} \mathbf{u}_{k, \ell}^{(\mu)}+\mathbf{f}_{k, \ell}, \quad \mu \in \mathbb{N}_{0}
$$

with any initial approximation $\mathbf{u}_{k, \ell}^{(0)}$.

Following theorem says that above iterative schemes are convergent for appropriately chosen $k$. 
Theorem 4.28 Assume that the operator $\mathcal{I}-\mathcal{K}$ is bijective on a Banach space $\mathbb{X}$ with $\mathcal{K}$ being compact on $\mathbb{X}$, and the sequence of projections $\mathcal{P}_{n}, n \in \mathbb{N}_{0}$ satisfies the nestedness property (4.120). Then, for each $\ell \geq 1$ and sufficiently large $k$, the iteration schemes (4.127) and (4.128) for solving the equation (4.116) are convergent.

Proof: This theorem is a generalization of Theorem 3.1 in [FMX]. We consider Banach space in this thesis instead of Hilbert space in [FMX], hence the projections are different. Recall the definition of operator $\mathcal{P}_{n}, n \in \mathbb{N}_{0}$ (3.52) and the property of pointwise convergence of $\mathcal{P}_{n}$ (3.53). By (4.119), we have for $i>j$,

$$
\lim _{n \rightarrow \infty}\left\|\left(\mathcal{P}_{n+i}-\mathcal{P}_{n+j}\right) \mathcal{K}\right\|=\lim _{n \rightarrow \infty}\left\|\mathcal{K}\left(\mathcal{P}_{n+i}-\mathcal{P}_{n+j}\right)\right\|=0
$$

Note that, for all $u \in \mathbb{X}$,

$$
\mathcal{U}_{k, \ell} u=\mathcal{U}_{k, \ell}\left(\mathcal{P}_{k+\ell}-\mathcal{P}_{k}\right) u
$$

and

$$
\mathcal{U}_{k, \ell} u=\mathcal{P}_{k} \mathcal{K}\left(\mathcal{P}_{k+\ell}-\mathcal{P}_{k}\right) u+\sum_{i=1}^{\ell-1} \mathcal{Q}_{k+i} \mathcal{K}\left(\mathcal{P}_{k+\ell}-\mathcal{P}_{k+i}\right) u .
$$

Hence, for each fixed $\ell \geq 1$,

$$
\left\|\mathcal{U}_{k, \ell}\right\| \leq C \sum_{j=0}^{\ell-1}\left\|\mathcal{K}\left(\mathcal{P}_{k+\ell}-\mathcal{P}_{k+j}\right)\right\| \rightarrow 0 \quad \text { as } \quad k \rightarrow \infty,
$$

where we have used the fact that $\left\|\mathcal{P}_{j}\right\| \leq C, j \geq 0$ and $\left\|\mathcal{Q}_{i}\right\| \leq C, i \geq 1$ (due to the pointwise convergence of $\mathcal{P}_{n}, n \in \mathbb{N}_{0}$ and the principle of uniformly boundedness). Similarly, we have

$$
\mathcal{L}_{k, \ell} u=\left(\mathcal{P}_{k+\ell}-\mathcal{P}_{k}\right) \mathcal{K} \mathcal{P}_{k} u+\sum_{i=0}^{\ell-1}\left(\mathcal{P}_{k+\ell}-\mathcal{P}_{k+i}\right) \mathcal{K} \mathcal{Q}_{k+1+i} u
$$

and thus

$$
\left\|\mathcal{L}_{k, \ell}\right\| \leq C \sum_{i=0}^{\ell-1}\left\|\left(\mathcal{P}_{k+\ell}-\mathcal{P}_{k+i}\right) \mathcal{K}\right\| \rightarrow 0 \quad \text { as } \quad k \rightarrow \infty .
$$

Moreover, since $\mathcal{U}_{k, \ell}+\mathcal{L}_{k, \ell}=\mathcal{K}_{k+\ell}-\mathcal{K}_{k}$,

$$
\left\|\mathcal{U}_{k, \ell}+\mathcal{L}_{k, \ell}\right\|=\left\|\left(\mathcal{P}_{k+\ell}-\mathcal{P}_{k}\right) \mathcal{K}\right\| \rightarrow 0 \quad \text { as } \quad k \rightarrow \infty
$$

On the other hand,

$$
\mathcal{D}_{k, \ell}^{-1}=\left(\mathcal{I}-\mathcal{K}_{k}\right)^{-1} \quad \text { and } \quad\left(\mathcal{D}_{k, \ell}-\mathcal{U}_{k, \ell}\right)^{-1}=\left(\mathcal{I}-\left(\mathcal{I}-\mathcal{K}_{k}\right)^{-1} \mathcal{U}_{k, \ell}\right)^{-1}\left(\mathcal{I}-\mathcal{K}_{k}\right)^{-1}
$$


Therefore,

$$
\left\|\mathcal{D}_{k, \ell}^{-1}\right\|=\left\|\left(\mathcal{I}-\mathcal{K}_{k}\right)^{-1}\right\| \quad \text { and } \quad\left\|\left(\mathcal{D}_{k, \ell}-\mathcal{U}_{k, \ell}\right)^{-1}\right\| \leq \frac{\left\|\left(\mathcal{I}-\mathcal{K}_{k}\right)^{-1}\right\|}{1-\left\|\left(\mathcal{I}-\mathcal{K}_{k}\right)^{-1}\right\|\left\|\mathcal{U}_{k, \ell}\right\|} .
$$

Since $\left\|\left(\mathcal{I}-\mathcal{K}_{k}\right)^{-1}\right\|$ is uniformly bounded for large enough $k$, in view of (4.129), these two inverses are both bounded uniformly for large enough $k$. Thus, for each fixed $\ell \geq 1$, the iteration operators for schemes (4.127) and (4.128) are both convergent to 0 in operator norm:

$$
\left\|\mathcal{D}_{k, \ell}^{-1}\left(\mathcal{L}_{k, \ell}+\mathcal{U}_{k, \ell}\right)\right\| \rightarrow 0 \quad \text { and } \quad\left\|\left(\mathcal{D}_{k, \ell}-\mathcal{U}_{k, \ell}\right)^{-1} \mathcal{L}_{k, \ell}\right\| \rightarrow 0 \quad \text { as } k \rightarrow \infty
$$

Therefore they can be less than 1 if $k$ is large enough, which in turn yields the convergence of the iteration schemes (4.127) and (4.128).

We now describe above iterative methods in terms of their matrix representations. The matrix representation of $(4.116)$ is

$$
\left(\mathbf{E}_{n}-\mathbf{K}_{n}\right) \mathbf{u}_{n}=\mathbf{f}_{n}
$$

Let $\mathbf{A}_{n}=\mathbf{E}_{n}-\mathbf{K}_{n}$. We begin with an initial level $k$ : $1 \leq k<n$ (we have seen, for the convergence of the iteration, $k$ needs to not too small), and let $\ell=n-k$ be the number of additional levels from level $k$ to the final level $n$. Then $\mathbf{A}_{n}$ is partitioned into $(\ell+1) \times(\ell+1)$ block matrix:

$$
\mathbf{A}_{n}=\left[\mathbf{A}_{i j}: i, j=k, . ., \ldots, k+\ell\right]
$$

Introduce following notations for the convenience of discussion:

$$
\begin{aligned}
& \mathbf{D}_{k, \ell}=\left[\begin{array}{cccc}
\mathbf{E}_{k}-\mathbf{K}_{k} & & & \\
& \mathbf{I} & & \\
& \ddots & \\
& & \mathbf{I}
\end{array}\right] \\
& \mathbf{U}_{k, \ell}=\left[\begin{array}{cccc}
O & \mathbf{E}_{k, k+1}-\mathbf{K}_{k, k+1} & \cdots & \mathbf{E}_{k, k+\ell}-\mathbf{K}_{k, k+\ell} \\
& O & \cdots & \mathbf{E}_{k+1, k+\ell}-\mathbf{K}_{k+1, k+\ell} \\
& & \ddots & \vdots
\end{array}\right]
\end{aligned}
$$


and

$$
\mathbf{L}_{k, \ell}=\left[\begin{array}{cccc}
O & & & \\
\mathbf{K}_{k+1, k} & \mathbf{K}_{k+1, k+1} & & \\
\vdots & \vdots & \ddots & \\
\mathbf{K}_{k+\ell, k} & \mathbf{K}_{k+\ell, k+1} & \cdots & \mathbf{K}_{k+\ell, k+\ell}
\end{array}\right]
$$

Accordingly, (4.132) is written as

$$
\left(\mathbf{D}_{k, \ell}+\mathbf{U}_{k, \ell}-\mathbf{L}_{k, \ell}\right) \mathbf{u}_{k, \ell}=\mathbf{f}_{k, \ell}
$$

The two multilevel iteration schemes (4.127) and (4.128) in terms of matrix representation now have forms:

- Jacobi type iteration:

$$
\mathbf{D}_{k, \ell} \mathbf{u}_{k, \ell}^{(\mu+1)}=\left(-\mathbf{U}_{k, \ell}+\mathbf{L}_{k, \ell}\right) \mathbf{u}_{k, \ell}^{(\mu)}+\mathbf{f}_{k, \ell}, \quad \mu \in \mathbb{N}_{0}
$$

with any initial guess $\mathbf{u}_{k, \ell}^{(0)}$. The iteration matrix for Jacobi type scheme is

$$
\mathbf{M}_{k, \ell}^{\mathrm{J}}=\mathbf{D}_{k, \ell}^{-1}\left(-\mathbf{U}_{k, \ell}+\mathbf{L}_{k, \ell}\right)
$$

- Gauss-Seidel type iteration:

$$
\left(\mathbf{D}_{k, \ell}+\mathbf{U}_{k, \ell}\right) \mathbf{u}_{k, \ell}^{(\mu+1)}=\mathbf{L}_{k, \ell} \mathbf{u}_{k, \ell}^{(\mu)}+\mathbf{f}_{k, \ell}, \quad \mu \in \mathbb{N}_{0}
$$

with any initial guess $\mathbf{u}_{k, \ell}^{(0)}$. The iteration matrix for Gauss-Seidel type scheme is:

$$
\mathbf{M}_{k, \ell}^{\mathrm{GS}}=\left(\mathbf{D}_{k, \ell}+\mathbf{U}_{k, \ell}\right)^{-1} \mathbf{L}_{k, \ell}
$$

Both (4.134) and (4.135) can be solved easily by "backward substitutions" for all blocks except the first block of $\mathbf{u}_{k, \ell}^{(\mu+1)}$. For the first block of $\mathbf{u}_{k, \ell}^{(\mu+1)}$, we need to find $\left(\mathbf{E}_{k}-\mathbf{K}_{k}\right)^{-1}$. In comparison with the Jacobi type iterate, Gauss-Seidel type scheme uses previously computed components within each iteration to compute the next component by backward substitution, while the Jacobi scheme doesn't use the new computed components until an iteration is completed. Both methods require inverting the submatrix $\mathbf{E}_{k}-\mathbf{K}_{k}$. Hence, we need to choose $k$ in order that the inverse of $\mathbf{E}_{k}-\mathbf{K}_{k}$ exists and is relatively easy to find, i.e, $k$ 


\begin{tabular}{|c|c|c|c|c|c|c|c|}
\hline & \multicolumn{4}{|c|}{ Multilevel iterate } & \multicolumn{3}{c|}{ Gaussian elimination } \\
$(k, \ell)$ & $\mu$ & $\left\|u_{k, \ell}^{(\mu)}-u\right\|_{\infty}$ & order & time(sec.) & $\left\|u_{k+\ell}^{*}-u\right\|_{\infty}$ & order & time(sec.) \\
\hline$(3,0)$ & & $7.499082 \mathrm{e}-3$ & & 0.16 & $7.499082 \mathrm{e}-3$ & & 0.16 \\
$(3,1)$ & 4 & $1.845814 \mathrm{e}-3$ & 2.0230 & 0.81 & $1.849162 \mathrm{e}-3$ & 2.0198 & 10 \\
$(3,2)$ & 8 & $4.650403 \mathrm{e}-4$ & 1.9888 & 8.74 & $4.650610 \mathrm{e}-4$ & 1.9914 & 632 \\
\hline
\end{tabular}

Table 4.14: Comparison of uncompressed solutions using Gaussian elimination and multilevel iteration methods.

is not too large (for example $k=2$ or 3 ). Conveniently, a good initial guess would be $\mathbf{u}_{k, \ell}^{(0)}:=\left[\left(\mathbf{E}_{k}-\mathbf{K}_{k}\right)^{-1} \mathbf{f}_{k}, 0, \ldots, 0\right]^{T}$. Another issue is that we need to choose a proper $k$ such that the iteration scheme is convergent. Namely, we need the spectral radius of $\mathbf{M}_{k, \ell}^{\mathrm{GS}}$ ( or $\left.\mathbf{M}_{k, \ell}^{\mathrm{J}}\right)$ is less than one. This can be achieved by requiring their matrix norms are less than one. Our experiments shows when the domain of integration is unit triangle $\Omega$, then $k$ must be $\geq 3$, and for other domains of integration (for example, a general triangle, a polygon), $k \geq 2$.

Example 4.29 In this example, we test if the Gauss-Seidel type multilevel iteration method gives good solution. The results are compared with the results from stand Gaussian elimination. All the entries are computed analytically and no compression is assumed. The results are tabulated in Table 4.14. We can see that both methods give very close solutions. Where, $\mu$ is the number of iterations. The significant reduction of the computing time is very impressive. 


\section{Chapter 5}

\section{Fast Wavelet Collocation Methods on Polygonal Domains}

\subsection{On General Triangles}

Let $\triangle$ denote a planar triangle in the $x y$-plane, and let vertices of $\triangle$ be denoted by $\left\{v_{j}, j \in\right.$ $\left.\mathbb{Z}_{3}\right\}$, where, $v_{j}:=\left(x_{j}, y_{j}\right)$. As before, $\Omega$ denotes the unit triangle, i.e.,

$$
\Omega=\{(s, t) \mid s, t \geq 0, s+t \leq 1\}
$$

The vertices of the unit triangle $\Omega$ are

$$
q_{0}=(0,0), \quad q_{1}=(1,0), \quad q_{2}=(0,1) .
$$

Define an affine mapping

$$
T: \Omega \underset{\text { onto }}{\stackrel{1-1}{\longrightarrow}} \triangle
$$

by

$$
(x, y)=T(s, t):=(1-s-t) v_{0}+s v_{1}+t v_{2},
$$

namely

$$
\left\{\begin{array}{l}
x=(1-s-t) x_{0}+s x_{1}+t x_{2} \\
y=(1-s-t) y_{0}+s y_{1}+t y_{2}
\end{array}\right.
$$


Note $T\left(q_{i}\right)=v_{i}, i \in \mathbb{Z}_{3}$. The inverse of this mapping is given by solving (5.3) for $(s, t)$, or equivalently by solving

$$
\left\{\begin{array}{l}
t\left(x_{2}-x_{0}\right)+s\left(x_{1}-x_{0}\right)=x-x_{0} \\
t\left(y_{2}-y_{0}\right)+s\left(y_{1}-y_{0}\right)=y-y_{0}
\end{array}\right.
$$

for $(s, t)$. This system is nonsingular since the vertices $\left\{v_{i}, i \in \mathbb{Z}_{3}\right\}$ are assumed not to be collinear. Denote the inverse mapping of $T$ by $T^{-1}$, then

$$
(s, t)=T^{-1}(x, y)
$$

$T^{-1}$ is an affine mapping too. Let

$$
A=\operatorname{det}\left(\begin{array}{cc}
x_{1}-x_{0} & x_{2}-x_{0} \\
y_{1}-y_{0} & y_{2}-y_{0}
\end{array}\right) .
$$

$T^{-1}$ has an explicit expression, namely,

$$
\left\{\begin{aligned}
s & =\frac{1}{A}\left[x\left(y_{2}-y_{0}\right)+y\left(x_{0}-x_{2}\right)+\left(x_{2} y_{0}-x_{0} y_{2}\right)\right] \\
t & =\frac{1}{A}\left[x\left(y_{0}-y_{1}\right)+y\left(x_{1}-x_{0}\right)+\left(x_{0} y_{1}-x_{1} y_{0}\right)\right]
\end{aligned}\right.
$$

Observe that the Jacobian of $T$ is $J_{T}=|A|$ and the Jacobian of $T^{-1}$ is $J_{T^{-1}}=\frac{1}{|A|}=\frac{1}{J_{T}}$. Also $J_{T}=2 \operatorname{Area}(\triangle)$.

Let $\mathbb{X}:=L^{\infty}(\triangle)$ and $\mathbb{V}:=C(\triangle)$. We now consider the integral equation

$$
u(x, y)-\int_{E} K(x, y, \xi, \eta) u(\xi, \eta) \mathrm{d} \xi \mathrm{d} \eta=f(x, y),
$$

where, $K(x, y, \xi, \eta)$ is a weakly singular kernel. $E$ here is the general triangle $\triangle$. Therefore the operator $\mathcal{K}: \mathbb{X} \rightarrow \mathbb{V}$ defined by

$$
(\mathcal{K} u)(x, y):=\int_{\triangle} K(x, y, \xi, \eta) u(\xi, \eta) \mathrm{d} \xi \mathrm{d} \eta, \quad(x, y) \in \triangle
$$

is compact in $\mathbb{X} . \quad u$ is the unknown in $\mathbb{X}$. Use the transformation $(x, y)=T(s, t),(5.9)$ changes into

$$
u(s, t)-J_{T} \int_{\Omega} K\left(T(s, t), T\left(s^{\prime}, t^{\prime}\right)\right) u\left(s^{\prime}, t^{\prime}\right) \mathrm{d} s^{\prime} \mathrm{d} t^{\prime}=f(T(s, t)) .
$$

Note that (5.11) is just an integral equation defined on the unit triangle, thus we can use the method described in Chapter 3 and Chapter 4 to solve (5.11). Assume the wavelet 


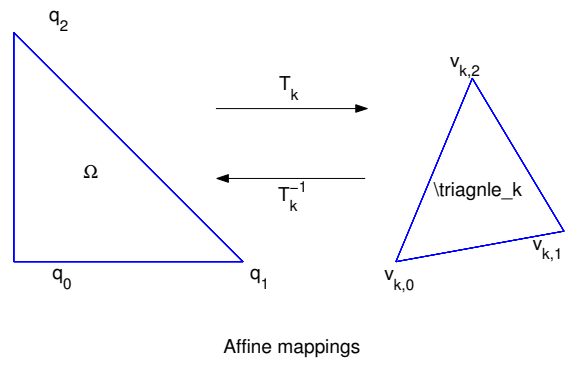

Figure 5.1: The affine mapping $T_{k}$

collocation solution to $(5.11)$ on $\Omega$ is $u_{n}(s, t)=\sum_{(i, j) \in \mathbb{U}_{n}} c_{i j} w_{i j}(s, t)$, then the collocation solution to $(5.9)$ on $\triangle$ can be obtained by

$$
u_{n}(x, y)=\sum_{(i, j) \in \mathbb{U}_{n}} c_{i j} w_{i j}\left(T^{-1}(x, y)\right)
$$

\subsection{On Polygons}

Given a polygonal domain $E$ in the plane $\mathbb{R}^{2}$, and let $\triangle_{\mathbf{p}}:=\left\{\triangle_{0}, \ldots, \triangle_{p-1}\right\}$ denote a triangulation of $E$. We assume triangles $\triangle_{j}$ and $\triangle_{k}\left(j, k \in \mathbb{Z}_{p}, j \neq k\right)$ can intersect only at vertices or along their common edges. Define affine mappings (see Fig. 5.1)

$$
T_{k}: \Omega \mapsto \triangle_{k} \quad k \in \mathbb{Z}_{p}
$$

Their expressions can be readily obtained from (5.3) or (5.4). Denote the three vertices of $\triangle_{k}$ by $\left\{v_{k, i}, i \in \mathbb{Z}_{3}\right\}$ with

$$
v_{k, i}=T_{k}\left(q_{i}\right), \quad i \in \mathbb{Z}_{3},
$$

where $v_{k, i}=\left(x_{k, i}, y_{k, i}\right)$.

We now develop the wavelet collocation method for the integral equation (5.9), but in this context, $E$ is a polygonal domain and $\mathbb{X}=L^{\infty}(E), \mathbb{V}=C(E)$. Note $E=\bigcup_{k=0}^{p-1} \triangle_{k}$. Observe that

$$
\Phi\left(T_{k}^{-1} \triangle_{k}\right)=T_{k}^{-1} \triangle_{k}, \quad k \in \mathbb{Z}_{p}
$$

where $\Phi=\left\{\phi_{e}, e \in \mathbb{Z}_{4}\right\}$ is defined as in (3.1). Hence we have

$$
T_{k} \circ \Phi \circ T_{k}^{-1} \triangle_{k}=\triangle_{k}
$$


For convenience, we define

$$
\phi_{k, e}:=T_{k} \circ \phi_{e} \circ T_{k}^{-1}, \quad k \in \mathbb{Z}_{p}, e \in \mathbb{Z}_{4}
$$

and

$$
\Phi_{k}:=T_{k} \circ \Phi \circ T_{k}^{-1}, \quad k \in \mathbb{Z}_{p}
$$

We see from (5.16), the set $\triangle_{k}, k \in \mathbb{Z}_{p}$ is the invariant set associated with the family of mappings $\Phi_{k}$. Define

$$
\hat{\Phi}:=\left\{\Phi_{k}: k \in \mathbb{Z}_{p}\right\}
$$

Then we readily obtain by (5.16) that

$$
\hat{\Phi}(E)=E .
$$

Namely, $E$ is an invariant set with respect to the family of contractive mappings $\hat{\Phi}$. We now determine the initial interpolating points for $\triangle_{k} k \in \mathbb{Z}_{p}$. Let

$$
G_{k, 0}=\left\{t_{0 j}^{(k)}: j \in \mathbb{Z}_{3}, k \in \mathbb{Z}_{p}\right\}
$$

with $t_{0 j}^{(k)}=T_{k} t_{0 j}, j \in \mathbb{Z}_{3}, k \in \mathbb{Z}_{p}$, and $t_{0 j} \in \Omega$ are defined in Chapter 3. Note $G_{k, 0}$ is refinable relative to the mappings $\Phi_{k}, k \in \mathbb{Z}_{p}$, i.e., $G_{k, 0}$ satisfies

$$
G_{k, 0} \subset \Phi_{k}\left(G_{k, 0}\right)
$$

Define $G_{0}:=\bigcup_{k \in \mathbb{Z}_{p}} G_{k, 0}$. Therefore, we obtain

$$
G_{0} \subset \hat{\Phi}\left(G_{0}\right)
$$

i.e., $G_{0}$ is refinable relatively with respect to the family of contractive mappings $\hat{\Phi}$. Now, we construct scaling functions and initial wavelets $w_{i j}^{(k)}, i \in \mathbb{Z}_{2}$ which are supported on $\triangle_{k}$ by

$$
w_{i j}^{(k)}(x, y)= \begin{cases}w_{i j} \circ T_{k}^{-1}(x, y) & (x, y) \in \triangle_{k} \\ 0 & (x, y) \in E \backslash \triangle_{k}\end{cases}
$$

where, when $i=0, j \in \mathbb{Z}_{3}$ and, when $i=1, j \in \mathbb{Z}_{9}$. Moreover, define

$$
\mathbb{W}_{0}=\operatorname{span}\left\{w_{0 j}^{(k)}: j \in \mathbb{Z}_{3}, k \in \mathbb{Z}_{p}\right\}
$$


and

$$
\mathbb{W}_{1}=\operatorname{span}\left\{w_{1 j}^{(k)}: j \in \mathbb{Z}_{9}, k \in \mathbb{Z}_{p}\right\}
$$

We now construct initial collocation functionals. Let

$$
\ell_{0 j}^{(k)}:=\delta_{t_{0 j}^{(k)}}, \text { with } t_{0 j}^{(k)}=T_{k}\left(t_{0 j}\right), \quad j \in \mathbb{Z}_{3}, k \in \mathbb{Z}_{p}
$$

and

$$
\ell_{1 j}^{(k)}=\sum_{l \in \mathbb{Z}_{12}} c_{j l}^{\prime} \delta_{t_{1 l}^{(k)}}, \text { with } t_{1 l}^{(k)}=T_{k}\left(t_{1 l}\right), \quad j \in \mathbb{Z}_{9}, k \in \mathbb{Z}_{p},
$$

where, $t_{1 l} \in \Omega, l \in \mathbb{Z}_{12}$ are defined in (3.30) of Chapter 3 , and $c_{j l}^{\prime}, j \in \mathbb{Z}_{9}, l \in \mathbb{Z}_{12}$ are elements of $\mathbf{C}^{\prime}$ in (3.33). In order to generate the multiscale wavelets for the space $\mathbb{W}_{i}, i \geq 2$, we define linear operators $\mathcal{T}_{k, e}: \mathbb{X}_{k} \rightarrow \mathbb{X}_{k}, e \in \mathbb{Z}_{4}, k \in \mathbb{Z}_{p}$ by

$$
\left(T_{k, e} x\right)(t):=x\left(\phi_{k, e}^{-1}(t)\right) \chi_{\phi_{k, e}\left(\triangle_{k}\right)}(t)
$$

where, $\mathbb{X}_{k}:=L^{\infty}\left(\triangle_{k}\right), k \in \mathbb{Z}_{p}$. For $\mathbf{e}:=\left(e_{0}, \ldots, e_{n-1}\right) \in \mathbb{Z}_{4}^{n}$ we introduce a composition operator $\mathcal{T}_{k, \mathbf{e}}$ by

$$
\mathcal{T}_{k, \mathbf{e}}:=\mathcal{T}_{k, e_{0}} \circ \ldots \circ \mathcal{T}_{k, e_{n-1}}
$$

Now, for $i=2,3, \ldots, n$, let

$$
w_{i j}^{(k)}(x, y)= \begin{cases}\mathcal{T}_{k, \mathbf{e}} w_{1 l}^{(k)}(x, y) & (x, y) \in \triangle_{k} \\ 0 & (x, y) \in E \backslash \triangle_{k},\end{cases}
$$

where, $j=9 \mu(\mathbf{e})+l, \mathbf{e} \in \mathbb{Z}_{4}^{i-1}, l \in \mathbb{Z}_{9}$. It follows that

$$
\mathbb{W}_{i}:=\operatorname{span}\left\{w_{i j}^{(k)}: j \in \mathbb{Z}_{w(i)}, k \in \mathbb{Z}_{p}\right\}
$$

Observe that the support of $w_{i j}^{(k)}$ is contained in $S_{i j}^{(k)}:=\phi_{k, \mathbf{e}}\left(\triangle_{k}\right), j \in \mathbb{Z}_{w(i)}$. To generate a multiscale collocation functionals, we introduce for any $e \in \mathbb{Z}_{4}$ a linear operator $\mathcal{L}_{k, e}: \mathbb{X}_{k}^{*} \rightarrow$ $\mathbb{X}_{k}^{*}$ defined by the equation

$$
\left\langle\mathcal{L}_{k, e} \ell, v\right\rangle=\left\langle\ell, v \circ \phi_{k, e}\right\rangle, \quad v \in \mathbb{X}_{k}, \ell \in \mathbb{X}_{k}^{*}
$$

Moreover, for $\mathbf{e}:=\left(e_{0}, \ldots, e_{n-1}\right) \in \mathbb{Z}_{4}^{n}$, we define the composite operator

$$
\mathcal{L}_{k, \mathbf{e}}:=\mathcal{L}_{k, e_{0}} \circ \ldots \circ \mathcal{L}_{k, e_{n-1}}
$$


Consequently, for any $\mathbf{e}, \mathbf{e}^{\prime} \in \mathbb{Z}_{4}^{i}, w \in \mathbb{X}_{k}$ and $\ell \in \mathbb{X}_{k}^{*}$, we have that

$$
\left\langle\mathcal{L}_{k, \mathbf{e}} l, \mathcal{T}_{k, \mathbf{e}^{\prime}} w\right\rangle=\langle l, w\rangle \delta_{\mathbf{e e}^{\prime}}
$$

Now, for $i>1, j=9 \mu(\mathbf{e})+l, \mathbf{e} \in \mathbb{Z}_{4}^{i-1}, l \in \mathbb{Z}_{9}$ define

$$
\ell_{i j}^{(k)}:=\mathcal{L}_{k, \mathbf{e}} \ell_{1 l}^{(k)}
$$

and observe that

$$
\left\langle\ell_{i j}^{(k)}, v\right\rangle=\left\langle\ell_{1 l}^{(k)}, v \circ \phi_{k, \mathbf{e}}\right\rangle=\sum_{s \in \mathbb{Z}_{12}} c^{\prime}{ }_{l s} v\left(\phi_{k, \mathbf{e}}\left(t_{1 s}^{(k)}\right)\right) .
$$

Note that the "support" of $\ell_{i j}^{(k)}$ is also contained in $S_{i j}^{(k)}$. We now discover some close ties between $\ell_{i j}^{(k)}, w_{i j}^{(k)}, k \in \mathbb{Z}_{p},(i, j) \in \mathbb{U}$ defined on $\triangle_{k}$ and $\ell_{i j}, w_{i j},(i, j) \in \mathbb{U}$ defined on $\Omega$. First observe that from (5.17), for any $\mathbf{e} \in \mathbb{Z}_{\mu}^{n}$ and $k \in \mathbb{Z}_{p}$,

$$
\phi_{k, \mathbf{e}}=T_{k} \circ \phi_{\mathbf{e}} \circ T_{k}^{-1}
$$

and

$$
T_{k}^{-1} \circ \phi_{k, e} \circ T_{k}=\phi_{e}, \quad T_{k}^{-1} \circ \phi_{k, \mathbf{e}} \circ T_{k}=\phi_{\mathbf{e}}
$$

Following two lemmas are important for the discussion of properties of $w_{i j}^{(k)}$ and $\ell_{i j}^{(k)}$.

\section{Lemma 5.1}

$$
\left\langle\ell_{i j}^{(k)}, v\right\rangle=\left\langle\ell_{i j}, v \circ T_{k}\right\rangle
$$

where, $v \in L_{\infty}\left(\triangle_{k}\right), k \in \mathbb{Z}_{p},(i, j) \in \mathbb{U}$.

Proof: For $j=\mu(\mathbf{e}) r+l, l \in \mathbb{Z}_{r}$, with $r=9$,

$$
\begin{aligned}
& \left\langle\ell_{i j}^{(k)}, v\right\rangle=\left\langle\mathcal{L}_{k, \mathbf{e}} \ell_{1 l}^{(k)}, v\right\rangle \\
= & \left\langle\ell_{1 l}^{(k)}, v \circ \phi_{k, \mathbf{e}}\right\rangle=\sum_{s \in \mathbb{Z}_{12}} c_{l s}^{\prime} v \circ \phi_{k, \mathbf{e}}\left(t_{1 s}^{(k)}\right) \\
= & \sum_{s \in \mathbb{Z}_{12}} c_{l s}^{\prime} v \circ \phi_{k, \mathbf{e}}\left(T_{k} t_{1 s}\right)=\sum_{s \in \mathbb{Z}_{12}} c_{l s}^{\prime} v \circ T_{k} \circ \phi_{\mathbf{e}} \circ T_{k}^{-1} \circ T_{k}\left(t_{1 s}\right) \\
= & \sum_{s \in \mathbb{Z}_{12}} c_{l s}^{\prime}\left(v \circ T_{k}\right) \circ \phi_{\mathbf{e}}\left(t_{1 s}\right)=\left\langle\ell_{i j}, v \circ T_{k}\right\rangle .
\end{aligned}
$$




\section{Lemma 5.2}

$$
w_{i j}^{(k)}(x, y)=w_{i j} \circ T_{k}^{-1}(x, y)
$$

Proof: For $j=\mu(\mathbf{e}) r+l, l \in \mathbb{Z}_{r}$, with $r=9$,

$$
\begin{aligned}
w_{i j}^{(k)}(x, y) & =\mathcal{T}_{k, \mathbf{e}} w_{1 l}^{(k)}(x, y) \\
& =w_{1 l}^{(k)} \circ \phi_{k, \mathbf{e}}^{-1}(x, y) \chi_{\phi_{k, \mathbf{e}}\left(\triangle_{k}\right)}(x, y) \\
& =\left(w_{1 l}^{(k)} \circ T_{k} \circ \phi_{\mathbf{e}}^{-1}\right) \circ T_{k}^{-1}(x, y) \chi_{T_{k} \circ \phi_{\mathbf{e}} \circ T_{k}^{-1}\left(\triangle_{k}\right)}(x, y) \\
& =\left(w_{1 l} \circ \phi_{\mathbf{e}}^{-1}\right) \circ T_{k}^{-1}(x, y) \chi_{T_{k} \circ \phi_{\mathbf{e}} \circ T_{k}^{-1}\left(\triangle_{k}\right)}(x, y) \\
& =\left(w_{1 l} \circ \phi_{\mathbf{e}}^{-1} \chi_{\phi_{\mathbf{e}}(\Omega)}\right) \circ T_{k}^{-1}(x, y) \\
& =w_{i j} \circ T_{k}^{-1}(x, y),
\end{aligned}
$$

where we have used $\chi_{T_{k} \phi_{\mathbf{e}} T_{k}^{-1}\left(\triangle_{k}\right)}(x, y)=\chi_{\phi_{\mathbf{e}}(\Omega)}(s, t)$ with $(x, y)=T_{k}(s, t),(x, y) \in \triangle_{k},(s, t) \in$ $\Omega$.

Remark: Lemma 5.1 and Lemma 5.2 provide alternative ways to construct $\ell_{i j}$ and $w_{i j}$ for $(i, j) \in \mathbb{U}_{n}, i \geq 2$ as compared to (5.31) and (5.36).

\subsection{Computation of Matrix Elements}

We now consider in detail the discretization of the integral equation on a polygonal domain E. Consider

$$
u(x, y)-\int_{E} K(x, y, \xi, \eta) \mathrm{d} \xi \mathrm{d} \eta=f(x, y),
$$

where $E$ is the polygonal domain, and $E$ has a triangulation $\triangle_{\mathbf{p}}$ such that $E=\cup_{k \in \mathbb{Z}_{p}} \triangle_{k}$. Let $T_{k}$ be the mapping associated with each triangle $\triangle_{k}$, such that

$$
(x, y)=T_{k}(s, t), \quad(x, y) \in \triangle_{k},(s, t) \in \Omega .
$$

Let $u_{n}$ be the approximation solution to $u$ in $\mathbb{F}_{n}$ with

$$
u_{n}(x, y)=\sum_{k \in \mathbb{Z}_{p}} \sum_{i, j} c_{i j}^{(k)} w_{i j}^{(k)}(x, y), \quad i \in \mathbb{Z}_{n+1}, j \in \mathbb{Z}_{w(i)}
$$


By using (5.45) we then have

$$
u_{n}(s, t)=\sum_{k \in \mathbb{Z}_{p}} \sum_{i, j} c_{i j}^{(k)} w_{i j}(s, t), \quad i \in \mathbb{Z}_{n+1}, j \in \mathbb{Z}_{w(i)}, \quad(s, t) \in \Omega
$$

Now use $u_{n}$ replacing $u$ in $(5.44)$, we have

$$
\begin{gathered}
\sum_{k^{\prime} \in \mathbb{Z}_{p}} \sum_{(i, j) \in \mathbb{U}_{n}} c_{i j}^{\left(k^{\prime}\right)} w_{i j}(s, t)-\sum_{k^{\prime} \in \mathbb{Z}_{p}} J_{T_{k^{\prime}}} \sum_{\substack{(i, j) \in \mathbb{U}_{n}\\
}} c_{i j}^{\left(k^{\prime}\right)} \int_{\Omega} K\left(T_{k}(s, t), T_{k^{\prime}}\left(s^{\prime}, t^{\prime}\right)\right) w_{i j}\left(s^{\prime}, t^{\prime}\right) \mathrm{d} s^{\prime} \mathrm{d} t^{\prime} \\
=f \circ T_{k}(s, t),
\end{gathered}
$$

where, $k \in \mathbb{Z}_{p}$. Now apply $\ell_{i^{\prime} j^{\prime}}^{(k)}, k \in \mathbb{Z}_{p},\left(i^{\prime}, j^{\prime}\right) \in \mathbb{U}_{n}$ to both sides of equation (5.48), we have

$$
\begin{aligned}
& \left\langle\ell_{i^{\prime} j^{\prime}}^{(k)}, \sum_{k^{\prime} \in \mathbb{Z}_{p}} \sum_{(i, j) \in \mathbb{U}_{n}} c_{i j}^{\left(k^{\prime}\right)} w_{i j}\right\rangle- \\
& \sum_{k^{\prime} \in \mathbb{Z}_{p}} J_{T_{k^{\prime}}} \sum_{(i, j) \in \mathbb{U}_{n}} c_{i j}^{\left(k^{\prime}\right)} \int_{\Omega}\left\langle\ell_{i^{\prime} j^{\prime}}^{(k)}, K\left(T_{k}(\cdot, \cdot), T_{k^{\prime}}\left(s^{\prime}, t^{\prime}\right)\right)\right\rangle w_{i j}\left(s^{\prime}, t^{\prime}\right) \mathrm{d} s^{\prime} \mathrm{d} t^{\prime}=\left\langle\ell_{i^{\prime} j^{\prime}}^{(k)}, f \circ T_{k}(s, t)\right\rangle
\end{aligned}
$$

After discretization, we obtain a system of linear equations

$$
\left(\mathbf{E}_{n}-\mathbf{K}_{n}\right) \mathbf{u}_{n}=\mathbf{f}_{n}
$$

where

$$
\begin{gathered}
\mathbf{E}_{n}=\left[E_{k^{\prime} i^{\prime} j^{\prime}, k i j}\right]=\left[\left\langle\ell_{i^{\prime} j^{\prime}}^{\left(k^{\prime}\right)}, w_{i j}^{(k)}\right\rangle: k, k^{\prime} \in \mathbb{Z}_{p},(i, j),\left(i^{\prime}, j^{\prime}\right) \in \mathbb{U}_{n}\right], \\
\mathbf{K}_{n}=\left[K_{k^{\prime} i^{\prime} j^{\prime}, k i j}: k, k^{\prime} \in \mathbb{Z}_{p},(i, j),\left(i^{\prime}, j^{\prime}\right) \in \mathbb{U}_{n}\right] \\
=\left[J_{T_{k}} \int_{\Omega}\left\langle\ell_{i^{\prime} j^{\prime}}^{\left(k^{\prime}\right)} K\left(T_{k^{\prime}}(\cdot, \cdot), T_{k}\left(s^{\prime}, t^{\prime}\right)\right)\right\rangle w_{i j}\left(s^{\prime}, t^{\prime}\right) \mathrm{d} s^{\prime} \mathrm{d} t^{\prime}: k, k^{\prime} \in \mathbb{Z}_{p},(i, j),\left(i^{\prime}, j^{\prime}\right) \in \mathbb{U}_{n}\right],
\end{gathered}
$$

and

$$
\mathbf{f}_{n}=\left[f_{k^{\prime} i j}\right]=\left[\left\langle\ell_{i^{\prime} j^{\prime}}^{\left(k^{\prime}\right)}, f T_{k^{\prime}}(s, t)\right\rangle: k^{\prime} \in \mathbb{Z}_{p},\left(i^{\prime}, j^{\prime}\right) \in \mathbb{U}_{n}\right]
$$

\subsection{Properties of the Wavelet Basis functions and the Collocation Functionals Over Polygons}

Next several lemmas tells us the vanishing moments and orthogonality of the initial collocation functionals $\ell_{i j}^{(k)}$ and wavelets $w_{i j}^{(k)}$, where $(i, j) \in \mathbb{U}_{2}, k \in \mathbb{Z}_{p}$. 


\section{Lemma 5.3}

$$
\left\langle\ell_{0 j}^{(k)}, w_{0 j^{\prime}}^{\left(k^{\prime}\right)}\right\rangle=\delta_{k k^{\prime}} \delta_{j j^{\prime}}, \quad j, j^{\prime} \in \mathbb{Z}_{3}, k \in \mathbb{Z}_{p}
$$

Proof: Clearly, when $k \neq k^{\prime},\left\langle\ell_{0 j}^{k}, w_{0 j^{\prime}}^{\left(k^{\prime}\right)}\right\rangle=0$ by the definition of $\ell_{0 j}^{(k)}$ and $w_{0 j^{\prime}}^{\left(k^{\prime}\right)}$. When $k=k^{\prime}$, $\left\langle\ell_{0 j}^{(k)}, w_{0 j^{\prime}}^{\left(k^{\prime}\right)}\right\rangle=w_{0 j^{\prime}}^{(k)}\left(t_{0 j}^{(k)}\right)=w_{0 j^{\prime}} \circ T_{k}^{-1} \circ T_{k}\left(t_{0 j}\right)=w_{0 j^{\prime}}\left(t_{0 j}\right)=\delta_{j^{\prime} j}$.

\section{Lemma 5.4}

$$
\left\langle\ell_{1 j}^{(k)}, w_{1 j^{\prime}}^{\left(k^{\prime}\right)}\right\rangle=\delta_{k k^{\prime}} \delta_{j j^{\prime}}, \quad j, j^{\prime} \in \mathbb{Z}_{9} ; \quad k, k^{\prime} \in \mathbb{Z}_{p}
$$

Proof: Clearly, when $k \neq k^{\prime},\left\langle\ell_{1 j}^{k}, w_{1 j^{\prime}}^{\left(k^{\prime}\right)}\right\rangle=0$ by the definition of $\ell_{1 j}^{(k)}$ and $w_{1 j^{\prime}}^{\left(k^{\prime}\right)}$. When $k=k^{\prime}$, $\left\langle\ell_{1 j}^{(k)}, w_{1 j^{\prime}}^{\left(k^{\prime}\right)}\right\rangle=\left\langle\ell_{1 j}^{(k)}, w_{1 j^{\prime}} \circ T_{k}^{-1}\right\rangle=\sum_{l \in \mathbb{Z}_{12}} c_{j l}^{\prime} w_{1 j^{\prime}} \circ T_{k}^{-1} \circ\left(T_{k} t_{1 l}\right)=\left\langle\ell_{1 j}, w_{1 j^{\prime}}\right\rangle=\delta_{j j^{\prime}}$.

\section{Lemma 5.5}

$$
\left\langle w_{1 j}^{(k)}, 1\right\rangle=0 . \quad j \in \mathbb{Z}_{9} ; \quad k \in \mathbb{Z}_{p} .
$$

Proof: $\left\langle w_{1 j}^{(k)}, 1\right\rangle=\int_{\triangle_{k}} w_{1 j}^{(k)} \cdot 1 \mathrm{~d} x \mathrm{~d} y=\int_{\triangle_{k}} w_{1 j} \circ T_{k}^{-1}(x, y) \mathrm{d} x \mathrm{~d} y=J_{T_{\triangle_{k}}} \int_{\Omega} w_{1 j} \circ T_{k}^{-1} \circ$ $T_{k}(s, t) \mathrm{d} s \mathrm{~d} t=0$.

\section{Lemma 5.6}

$$
\left\langle w_{1 j}^{(k)}, x\right\rangle=0
$$

and

$$
\left\langle w_{1 j}^{(k)}, y\right\rangle=0
$$

where, $j \in \mathbb{Z}_{9}, k \in \mathbb{Z}_{p}$.

Proof: $\left\langle w_{1 j}^{(k)}, x\right\rangle=\int_{\triangle_{k}} w_{1 j}^{(k)} x \mathrm{~d} x \mathrm{~d} y=\int_{\triangle_{k}} w_{1 j} \circ T_{k}^{-1}(x, y) x \mathrm{~d} x \mathrm{~d} y=J_{T_{k}} \int_{\Omega} w_{1 j} \circ T_{k}^{-1} \circ T_{k}(s, t)[(1-$ $\left.s-t) x_{k, 0}+s x_{k, 1}+t x_{k, 2}\right] \mathrm{d} s \mathrm{~d} t=0$ by the vanishing moment property of $w_{1 j}$ on $\Omega$ (Lemma $3.3)$.

Similarly, we can obtain $\left\langle w_{1 j}^{(k)}, y\right\rangle=0$. 


\section{Lemma 5.7}

$$
\left\langle\ell_{1 j}^{(k)}, 1\right\rangle=0, \quad j \in \mathbb{Z}_{9} ; \quad k \in \mathbb{Z}_{p}
$$

Proof: $\left\langle\ell_{1 j}^{(k)}, 1\right\rangle=\left\langle\sum_{l \in \mathbb{Z}_{12}} c_{j l}^{\prime} \delta_{t_{1 l}^{(k)}}, 1\right\rangle=\sum_{l \in \mathbb{Z}_{12}} c_{j l}^{\prime}\left\langle T_{k}\left(t_{1 l}\right), 1\right\rangle=\sum_{l \in \mathbb{Z}_{12}} c_{j l}^{\prime}=0$ by Lemma 3.3.

\section{Lemma 5.8}

$$
\left\langle\ell_{1 j}^{(k)}, x\right\rangle=0
$$

and

$$
\left\langle\ell_{1 j}^{(k)}, y\right\rangle=0
$$

where $j \in \mathbb{Z}_{9}$ and $k \in \mathbb{Z}_{p}$.

Proof: First note $\left\langle\ell_{1 j}^{(k)}, x\right\rangle=\sum_{l \in \mathbb{Z}_{12}} c_{j l}^{\prime}\left\langle\delta_{T_{k}\left(t_{1 l}\right)}, x\right\rangle$. Now define $g(x, y):=x=(1-s-t) x_{k, 0}+$ $s x_{k, 1}+t x_{k, 2}:=h(s, t)$, where $(s, t) \in \Omega$ and $(x, y) \in \triangle_{k}$. Thus, we have

$$
g(x, y)=h \circ T_{k}^{-1}(x, y) .
$$

Therefore,

$\sum_{l \in \mathbb{Z}_{12}} c_{j l}^{\prime}\left\langle\delta_{T_{k}\left(t_{1 l}\right)}, x\right\rangle=\sum_{l \in \mathbb{Z}_{12}} c_{j l}^{\prime}\left\langle\delta_{T_{k}\left(t_{1 l}\right)}, h \circ T_{k}^{-1}\right\rangle=\sum_{l \in \mathbb{Z}_{12}} c_{j l}^{\prime} h \circ T_{k}^{-1} \circ\left(T_{k} t_{1 l}\right)=\sum_{l \in \mathbb{Z}_{12}} c_{j l}^{\prime} h\left(t_{1 l}\right)=$ $\left\langle\ell_{1 j}, h\right\rangle=0$ by the vanishing moment of $\ell_{1 j}$ (Lemma 3.3) since $h(s, t)$ is linear in $s, t$. Similarly, we can prove $\left\langle\ell_{1 j}^{(k)}, y\right\rangle=0$.

Lemma 5.9 For matrix $\mathbf{E}_{n}:=\left[E_{k^{\prime} i^{\prime} j^{\prime}, k i j}\right]$, where $i^{\prime}, i \in \mathbb{Z}_{n+1}, j \in \mathbb{Z}_{w(i)}, j^{\prime} \in \mathbb{Z}_{w\left(i^{\prime}\right)}, k, k^{\prime} \in$ $\mathbb{Z}_{p}$

if $k \neq k^{\prime}, E_{k^{\prime} i^{\prime} j^{\prime}, k i j}=0$. For $k=k^{\prime}$,

if $i^{\prime}>i, E_{k, i^{\prime} j^{\prime}, k i j}=0$

if $i^{\prime}=i, E_{k i^{\prime} j^{\prime}, k i j}=\delta_{i^{\prime} i} \delta_{j j^{\prime}}$

if $i^{\prime}<i$, in each submatrix $\mathbf{E}_{k i^{\prime}, k i}$ with shape parameter $\theta$, only the elements on the main diagonal line are possibly nonzero (cf. Lemma 4.3). 
Proof: When $k \neq k^{\prime}$, by the definition of the wavelet $w_{i j}^{(k)}$ and collocation functional $\ell_{i^{\prime} j^{\prime}}^{\left(k^{\prime}\right)}$, $E_{k^{\prime} i^{\prime} j^{\prime}, k i j}=0$. When $k=k^{\prime}$ the proof is parallel to the proof of Lemma 4.3.

Lemma 5.10 through Lemma 5.17 tells us important properties of the constructed wavelet basis functions and the collocation functionals on polygons. They are essentially the same properties as those of the wavelet basis functions and the collocation functionals on the unit triangle.

Lemma 5.10 The constructed wavelet basis functions $w_{i j}^{(k)}$ over the polygon E are locally supported and their supports are shrinking as level $i$ increases.

Proof: for every $i>1$, the support of $w_{i j}^{(k)}$ is contained in $S_{i j}^{(k)}=\phi_{k, \mathbf{e}}\left(\triangle_{k}\right)=\left(\triangle_{k}\right)_{i-1, \mu(\mathbf{e})}$, where $j=\mu(\mathbf{e}) r+l, l \in \mathbb{Z}_{r}$, with $r=9$.

Lemma 5.11 For any $i, i^{\prime} \in \mathbb{N}_{0}$,

$$
\begin{gathered}
\left\langle\ell_{i^{\prime} j^{\prime}}^{\left(k^{\prime}\right)}, w_{i j}^{(k)}\right\rangle=\delta_{k^{\prime} k} \delta_{i i^{\prime}} \delta_{j j^{\prime}}, \quad(i, j),\left(i^{\prime}, j^{\prime}\right) \in \mathbb{U}, i \leq i^{\prime}, k, k^{\prime} \in \mathbb{Z}_{p} \\
\sum_{j \in \mathbb{Z}_{w(i)}}\left|\left\langle\ell_{i^{\prime} j^{\prime}}^{(k)}, w_{i j}^{(k)}\right\rangle\right| \leq \frac{81}{28}, \quad(i, j),\left(i^{\prime}, j^{\prime}\right) \in \mathbb{U}, i>i^{\prime}
\end{gathered}
$$

and if $\gamma:=\frac{81}{28}$, then

$$
\gamma<\mu^{s / 2}-1, \quad \text { with } \quad s=2
$$

where, $s$ is the order of piecewise polynomials in the approximation spaces.

Proof: Lemma 5.9 proves (5.60). We have proved

$$
\sum_{j \in \mathbb{Z}_{w(i)}}\left|\left\langle\ell_{i^{\prime} j^{\prime}}, w_{i j}\right\rangle\right| \leq \frac{81}{28}, \quad(i, j),\left(i^{\prime}, j^{\prime}\right) \in \mathbb{U}, i>i^{\prime}
$$

in Lemma 3.2. In view of Lemma 5.1 and Lemma 5.2, we have

$$
\left\langle\ell_{i^{\prime} j^{\prime}}^{(k)}, w_{i j}^{(k)}\right\rangle=\left\langle\ell_{i^{\prime} j^{\prime}}, w_{i j}\right\rangle
$$

Thus

$$
\sum_{j \in \mathbb{Z}_{w(i)}}\left|\left\langle\ell_{i^{\prime} j^{\prime}}^{(k)}, w_{i j}^{(k)}\right\rangle\right| \leq \frac{81}{28}, \quad(i, j),\left(i^{\prime}, j^{\prime}\right) \in \mathbb{U}, i>i^{\prime}
$$


Direct computation shows (5.62).

Lemma 5.12 The constructed wavelets and collocation functionals have vanishing moment.

Proof: From Lemma 5.7 and Lemma 5.8, we obtain

$$
\left\langle\ell_{i^{\prime} j^{\prime}}^{(k)}, w_{0 j}^{(k)}\right\rangle=\left\langle\ell_{1 l^{\prime}}^{(k)}, w_{0 j}^{(k)} \circ \phi_{k, \mathbf{e}^{\prime}}\right\rangle=0, \quad j \in \mathbb{Z}_{3},
$$

where, $i^{\prime} \geq 2, j^{\prime} \in \mathbb{Z}_{w\left(i^{\prime}\right)}$ and $j^{\prime}=9 \mu\left(\mathbf{e}^{\prime}\right)+l^{\prime}, l^{\prime} \in \mathbb{Z}_{9}$ with $\mathbf{e}^{\prime} \in \mathbb{Z}_{4}^{i^{\prime}-1}$. We now consider $\mathcal{T}_{k, \mathbf{e}}$ as an operator from $L^{2}\left(\triangle_{k}\right)$ to $L^{2}\left(\triangle_{k}\right)$ and Let $\mathcal{T}_{k, \mathbf{e}}^{*}$ be the adjoint of $\mathcal{T}_{k, \mathbf{e}}$. It is easy to see that for $y \in L^{2}\left(\triangle_{k}\right)$

$$
\mathcal{T}_{k, \mathbf{e}}^{*} y=J_{\phi_{k, \mathbf{e}}} y \circ \phi_{k, \mathbf{e}}
$$

where $J_{\phi_{k, \mathbf{e}}}$ is the Jacobian of mapping $\phi_{k, \mathbf{e}}$. Therefore, we have that

$$
\left(w_{i j}^{(k)}, w_{0 j^{\prime}}^{(k)}\right)=\left(\mathcal{T}_{k, \mathbf{e}} w_{1 l}^{(k)}, w_{0 j^{\prime}}^{(k)}\right)=\left(w_{1 l}^{(k)}, \mathcal{T}_{k, \mathbf{e}}^{*} w_{0 j^{\prime}}^{(k)}\right)=0
$$

where, $i \geq 2, j \in \mathbb{Z}_{w(i)}$ and $j=9 \mu(\mathbf{e})+l, l \in \mathbb{Z}_{9}$ with $\mathbf{e} \in \mathbb{Z}_{4}^{i-1}$. The last equality holds because $\mathcal{T}_{k, \mathbf{e}}^{*} w_{0 j^{\prime}}^{(k)}$ is a polynomial of total degree $\leq s-1=1$, with $s=2$ and $w_{1 l}^{(k)}$ satisfies Lemma 5.5 and Lemma 5.6. Thus for any polynomials $p \in \pi_{2}$,

$$
\left\langle\ell_{i j}^{(k)}, p\right\rangle=0, \quad\left(w_{i j}^{k}, p\right)=0, \quad(i, j) \in \mathbb{U}, i \geq 1 .
$$

Lemma 5.13 Both basis functions and collocation functionals are uniformly bounded.

Proof: For $(i, j) \in \mathbb{U}, i \geq 2, j=\mu(\mathbf{e}) r+l, l \in \mathbb{Z}_{9}$, Lemma 5.1 and Lemma 3.4 yields

$$
\left|\left\langle\ell_{i j}^{(k)}, v\right\rangle\right|=\left|\left\langle\ell_{i j}, v \circ T_{k}\right\rangle\right|=\left|\left\langle\ell_{1 l}, v \circ T_{k} \circ \phi_{\mathbf{e}}\right\rangle\right| \leq\left\|\mathbf{C}^{\prime}\right\|_{\infty}\|v\|_{\infty}
$$

and Lemma 5.2 yields

$$
\left\|w_{i j}^{(k)}\right\|_{\infty}=\left\|w_{i j} T_{k}^{-1}\right\|_{\infty} \leq\|\mathbf{C}\|_{\infty} \max _{j \in \mathbb{Z}_{12}}\left\|\psi_{j}\right\|_{\infty}
$$

The last inequality holds because of Lemma 3.4. 
Lemma 5.14 The dimension of spaces $\mathbb{F}_{n}$ and $\mathbb{W}_{n}$ grows exponentially in $n$ and the diameters $d_{n}$ decay exponentially in $n$ too, where

$$
d_{n}:=\max \left\{\mathrm{d}\left(S_{n m}^{(k)}\right),(n, m) \in \mathbb{U}, k \in \mathbb{Z}_{p}\right\}
$$

Proof: $\operatorname{dim} \mathbb{W}_{0}=3 p, \operatorname{dim} \mathbb{F}_{n}=3 p .4^{n}, n \in \mathbb{Z}_{0}$ and $\operatorname{dim} \mathbb{W}_{n}=9 p .4^{n-1}, n \geq 1, n \in \mathbb{Z}$.

Let $\hat{d}=\max \left\{d^{(k)}, k \in \mathbb{Z}_{p}\right\}$, where $d^{(k)}$ is the diameter of each triangle $\triangle_{k}, k \in \mathbb{Z}_{p}$ in the triangulation $\triangle_{\mathbf{p}}$. For the diameter we have $d_{0}=\hat{d}$ and $d_{n} \leq c \cdot \hat{d} \frac{1}{2^{n-1}}=c 2^{-n}$, where $c>0$ is some constant.

Lemma 5.15 There exists constant $\theta_{2}$ and $\theta_{3}$ such that for all $n \in \mathbb{N}_{0}$ and $v$ having form

$$
\begin{gathered}
v:=\sum_{(i, j) \in \mathbb{U}_{n}} v_{k, i j} w_{i j}^{(k)}, \quad k \in \mathbb{Z}_{p}, \\
\theta_{2}\|\mathbf{v}\|_{\infty} \leq\|v\|_{\infty} \leq \theta_{3}(n+1)\left\|\mathbf{E}_{n} \mathbf{v}\right\|_{\infty},
\end{gathered}
$$

where $\mathbf{v}:=\left[v_{k, i j}:(i, j) \in \mathbb{U}_{n}, k \in \mathbb{Z}_{p}\right]^{T}$.

Proof: Note the constructed wavelets $w_{i j}^{(k)}$ and collocation functionals $\ell_{i^{\prime} j^{\prime}}^{(k)}$, with $(i, j),\left(i^{\prime}, j^{\prime}\right) \in$ $\mathbb{U}, k \in \mathbb{Z}_{p}$ have properties stated in Lemma 5.10 - Lemma 5.13, the proof is then similar to the proof of Lemma 3.6.

Lemma 5.16 Let $\mathcal{P}_{n}$ be the projection operator from $\mathbb{X}$ onto $\mathbb{F}_{n}$ defined by

$$
\left\langle\ell_{i j}^{(k)}, \mathcal{P}_{n} x\right\rangle=\left\langle\ell_{i j}^{(k)}, x\right\rangle,(i, j) \in \mathbb{U}_{n}
$$

For $v \in L^{\infty}(E)$ we set

$$
\mathcal{P}_{n} v=\sum_{(i, j) \in \mathbb{U}_{n}} v_{k, i j} w_{i j}^{(k)}, \quad k \in \mathbb{Z}_{p} .
$$

Then, for any $x \in L^{\infty}(E)$, there holds

$$
\lim _{n \rightarrow \infty}\left\|\mathcal{P}_{n} x-x\right\|_{\infty}=0
$$

Proof: The proof is similar to that of Lemma 3.7. 
Lemma 5.17 There exists a positive constant c such that for $u \in W^{k, \infty}(E)$

$$
\operatorname{dist}\left(u, \mathbb{F}_{n}\right) \leq c \mu^{-k n / 2}\|u\|_{k, \infty}
$$

Proof: The proof is similar to that of Lemma 3.8 .

Theorem 5.18 If we use the constructed wavelet basis $w_{i j}^{(k)}$ and collocation functionals $\ell_{i j}^{(k)}$ on a polygon solve (2.2) with the spacial domain being a polygon, then Theorem 4.13, Theorem 4.14, Theorem 4.15, and Theorem 4.16 continue to hold.

Proof: The theorem follows from the properties Lemma 5.10 through 5.17 of the constructed wavelet basis functions and collocation functionals over polygons. 


\section{Chapter 6}

\section{Numerical Experiments for Solving Integral Equations}

In this chapter, we use methods proposed in this thesis to solve several typical examples. As far as compression strategies are concerned, we use both distance computing strategy 2 and block truncation strategy. The block truncation strategy manifests good truncation effect in terms of presenting satisfactory results. The resulting linear systems are solved by Gauss-Seidel iterative scheme developed in Section 4.8. When level $n$ is relatively large (say $n \geq 6$ ), the iterative scheme dramatically reduce the computing time by comparing with Gaussian elimination method. When the matrix entries are computed numerically, the quadrature rule presented in Section 4.3 is adopted. All the computation are done using parallel computing algorithm, which is discussed in Section 6.4.

\subsection{On the Unit Triangle}

Consider following integral equation of the second kind

$$
u(x)-\int_{\Omega} \frac{1}{|x-y|} u(y) \mathrm{d} y=f(x),
$$


Compression strategy: strategy 2 with $\nu=1$

\begin{tabular}{|c|c|c|c|c|c|c|}
\hline & \multicolumn{2}{|c|}{ without quadrature } & \multicolumn{4}{|c|}{ with quadrature } \\
$n$ & $L^{\infty}$-error & order & $\left(m_{1}, m_{2}\right)$ & $L^{\infty}$-error & order & compression rate \\
\hline 1 & $1.513187 \mathrm{e}-1$ & & $(40,40)$ & $1.513184 \mathrm{e}-1$ & & 1.000000 \\
2 & $3.599605 \mathrm{e}-2$ & 2.0717 & $(40,40)$ & $3.599379 \mathrm{e}-2$ & 2.0718 & 1.000000 \\
$(3,0)$ & $7.499082 \mathrm{e}-3$ & 2.2631 & $(80,40)$ & $7.498160 \mathrm{e}-3$ & 2.2631 & 1.000000 \\
$(3,1)$ & $1.849142 \mathrm{e}-3$ & 2.0199 & $(80,40)$ & $1.849507 \mathrm{e}-3$ & 2.0194 & 0.526763 \\
$(3,2)$ & $4.644798 \mathrm{e}-4$ & 1.9932 & $(80,40)$ & $4.646119 \mathrm{e}-4$ & 1.9930 & 0.228013 \\
$(3,3)$ & $1.168886 \mathrm{e}-4$ & 1.9905 & $(80,40)$ & $1.168323 \mathrm{e}-4$ & 1.9916 & 0.085760 \\
$(3,4)$ & $2.955001 \mathrm{e}-5$ & 1.9839 & $(80,40)$ & $2.982907 \mathrm{e}-5$ & 1.9696 & 0.030163 \\
$(3,5)$ & $1.081540 \mathrm{e}-5$ & 1.4501 & $(80,40)$ & $1.082801 \mathrm{e}-5$ & 1.4620 & 0.010460 \\
\hline
\end{tabular}

Table 6.1: Comparison of compressed solutions using multilevel iteration method, with and without quadrature rule, number of iteration $=15$.

where $x, y \in \mathbb{R}^{2}, x=\left(x_{1}, x_{2}\right), y=\left(y_{1}, y_{2}\right)$, and $\Omega$ is the unit triangle.

We choose

$$
f(x)=x_{1}^{2}+x_{2}^{2}-\int_{\Omega} \frac{y_{1}^{2}+y_{2}^{2}}{|x-y|} \mathrm{d} y
$$

so that the exact solution to $(6.1)$ is

$$
u(x)=x_{1}^{2}+x_{2}^{2}
$$

Example 6.1 In this example, we apply compression strategy 2 and use Gauss-Seidel type multilevel iteration method to solve the resulting linear system. The initial level $k$ used for the multilevel iteration is chosen as $k=3$. Note when the level $n=k+\ell=3+5=8$, the matrix size is $196608 \times 196608$, and the compression rate is about $1 \%$. It's surprising that even the matrix entries are computed without quadrature (computed by analytical formula. Only when the domain of integration is unit triangle, can we find the explicit formula by Mathematica. When the domain of integration is a general triangle, no explicit formula is available), for $n=8$, the result is far from satisfactory one. This shows the limitation of strategy 2. 
Compression strategy: block truncation with $p_{i^{\prime} i}=\sqrt{3}$

\begin{tabular}{|c|c|c|c|c|c|c|}
\hline & \multicolumn{2}{|c|}{ without quadrature } & \multicolumn{5}{|c|}{ with quadrature } \\
$n$ & $L^{\infty}$-error & order & $\left(m_{1}, m_{2}\right)$ & $L^{\infty}$-error & order & compression rate \\
\hline 1 & $1.513187 \mathrm{e}-1$ & & $(40,40)$ & $1.513184 \mathrm{e}-1$ & & 1.000000 \\
2 & $3.599605 \mathrm{e}-2$ & 2.0717 & $(40,40)$ & $3.599379 \mathrm{e}-2$ & 2.0718 & 1.000000 \\
$(3,0)$ & $7.499082 \mathrm{e}-3$ & 2.2631 & $(80,40)$ & $7.499123 \mathrm{e}-3$ & 2.2630 & 1.000000 \\
$(3,1)$ & $1.850492 \mathrm{e}-3$ & 2.0188 & $(80,40)$ & $1.850509 \mathrm{e}-3$ & 2.0188 & 0.431732 \\
$(3,2)$ & $4.648565 \mathrm{e}-4$ & 1.9931 & $(80,40)$ & $4.648294 \mathrm{e}-4$ & 1.9931 & 0.181965 \\
$(3,3)$ & $1.171548 \mathrm{e}-4$ & 1.9884 & $(80,40)$ & $1.174169 \mathrm{e}-4$ & 1.9851 & 0.067760 \\
$(3,4)$ & $2.999366 \mathrm{e}-5$ & 1.9657 & $(80,40)$ & $2.980252 \mathrm{e}-5$ & 1.9781 & 0.023004 \\
$(3,5)$ & $8.010510 \mathrm{e}-6$ & 1.9047 & $(80,40)$ & $7.920534 \mathrm{e}-6$ & 1.9118 & 0.007332 \\
\hline
\end{tabular}

Table 6.2: Comparison of compressed solutions using multilevel iteration method, with and without quadrature rule, number of iteration $=15$.

Example 6.2 In this example, we apply block truncation strategy and use Gauss-Seidel type multilevel iteration method to solve the resulting linear system. The initial level $k$ used for the multilevel iteration is chosen as $k=3$. The block truncation parameter $p_{i^{\prime} i}=\sqrt{3}, i^{\prime}, i \in$ $\mathbb{Z}_{n+1}$. Note compression rates in this case are even smaller than their counterparts using strategy 2. What's most amazing is that when $n=8$, the block truncation scheme continues to give satisfied solution. This suggests block truncation scheme is superior to strategy 2 in order to catch critical elements.

\subsection{On General Triangles}

We consider the triangle $\triangle$ with three vertices $(0,0),(1,0)$ and $(0.5,1)$. As before, we take the kernel

$$
K(x, y, \xi, \eta)=\frac{1}{\sqrt{(x-\xi)^{2}+(y-\eta)^{2}}}, \quad(x, y),(\xi, \eta) \in \triangle
$$

and

$$
f(x, y)=x^{2}+y^{2}-\int_{\triangle} \frac{\xi^{2}+\eta^{2}}{\sqrt{(x-\xi)^{2}+(y-\eta)^{2}}} \mathrm{~d} \xi \mathrm{d} \eta, \quad(x, y) \in \triangle
$$


Results for the general triangle $\triangle$ : strategy 2 with $\nu=1$

\begin{tabular}{|c|c|c|c|c|c|c|}
\hline & \multicolumn{2}{|c|}{ full collocation solution $u_{n}$} & \multicolumn{4}{|c|}{ compressed solution $\tilde{u}_{n}$} \\
\hline$n$ & $L^{\infty}$-error & order & $\left(m_{1}, m_{2}\right)$ & $L^{\infty}$-error & order & compression rate \\
\hline 1 & $1.100676 \mathrm{e}-1$ & & $(40,40)$ & $1.100676 \mathrm{e}-1$ & & 1.000000 \\
$(2,0)$ & $2.081240 \mathrm{e}-2$ & 2.4029 & $(80,40)$ & $2.081280 \mathrm{e}-2$ & 2.4028 & 1.000000 \\
$(2,1)$ & $4.972866 \mathrm{e}-3$ & 2.0653 & $(80,40)$ & $4.973775 \mathrm{e}-3$ & 2.0651 & 0.894531 \\
$(2,2)$ & $1.285098 \mathrm{e}-3$ & 1.9522 & $(80,40)$ & $1.286323 \mathrm{e}-3$ & 1.9511 & 0.520172 \\
$(2,3)$ & $3.230465 \mathrm{e}-4$ & 1.9921 & $(80,40)$ & $3.232655 \mathrm{e}-4$ & 1.9925 & 0.227601 \\
$(2,4)$ & & & $(80,40)$ & $7.800080 \mathrm{e}-5$ & 2.0512 & 0.085734 \\
$(2,5)$ & & & $(80,40)$ & $2.100672 \mathrm{e}-5$ & 1.8926 & 0.030162 \\
$(2,6)$ & & & $(80,40)$ & $5.914368 \mathrm{e}-6$ & 1.8286 & 0.010460 \\
\hline
\end{tabular}

Table 6.3: Comparison of solutions obtained by the full collocation method and by our compressed collocation method (strategy 2, on a general triangle).

so that $u(x, y)=x^{2}+y^{2},(x, y) \in \triangle$ is the exact solution to equation (5.9). In this case, no analytical formula is available for computing the matrix entry. All entries are computed by quadrature method proposed in Section 4.3. For the uncompressed solution, the linear system is solved by stand Gaussian elimination method, and the number of subdivision $m$ used in the quadrature is taken as $m=40$. While, for the compressed solution, the linear system is solved by Gauss-Seidel type iterative method presented in Section 4.8 (number of iteration $=20$ ), and the number of subdivision used in quadrature is as shown in Table 6.3 and Table 6.4.

Example 6.3 In this example, we use the truncation strategy 2 with $\nu=1$. The computational results are listed in Table 6.3 .

Example 6.4 In this example, we use the block truncation strategy with parameters $p_{i^{\prime} i}=$ $\sqrt{3}$. The results are listed in Table 6.4 . 
Results for the general triangle $\triangle$ : block truncation with $p_{i^{\prime} i}=\sqrt{3}$

\begin{tabular}{|c|c|c|c|c|c|c|}
\hline & \multicolumn{2}{|c|}{ full collocation solution $u_{n}$} & \multicolumn{4}{|c|}{ compressed solution $\tilde{u}_{n}$} \\
\hline$n$ & $L^{\infty}$-error & order & $\left(m_{1}, m_{2}\right)$ & $L^{\infty}$-error & order & compression rate \\
\hline 1 & $1.100676 \mathrm{e}-1$ & & $(40,40)$ & $1.100676 \mathrm{e}-1$ & & 1.000000 \\
$(2,0)$ & $2.081240 \mathrm{e}-2$ & 2.4029 & $(80,40)$ & $2.081280 \mathrm{e}-2$ & 2.4028 & 1.000000 \\
$(2,1)$ & $4.972866 \mathrm{e}-3$ & 2.0653 & $(80,40)$ & $4.974875 \mathrm{e}-3$ & 2.0647 & 0.758301 \\
$(2,2)$ & $1.285098 \mathrm{e}-3$ & 1.9522 & $(80,40)$ & $1.285873 \mathrm{e}-3$ & 1.9519 & 0.416626 \\
$(2,3)$ & $3.230465 \mathrm{e}-4$ & 1.9921 & $(80,40)$ & $3.257940 \mathrm{e}-4$ & 1.9807 & 0.181021 \\
$(2,4)$ & & & $(80,40)$ & $7.869679 \mathrm{e}-5$ & 2.0496 & 0.067701 \\
$(2,5)$ & & & $(80,40)$ & $2.195469 \mathrm{e}-5$ & 1.8418 & 0.023000 \\
$(2,6)$ & & & $(80,40)$ & $7.829137 \mathrm{e}-6$ & 1.4876 & 0.007331 \\
\hline
\end{tabular}

Table 6.4: Comparison of solutions obtained by the full collocation method and by our compressed collocation method (block truncation, on a general triangle).

\subsection{On Polygons}

In the following examples, as before, we take kernel as $K(x, y, \xi, \eta)=\frac{1}{\sqrt{(x-\xi)^{2}+(y-\eta)^{2}}}$, and $f(x, y)=x^{2}+y^{2}-\int_{E} \frac{\xi^{2}+\eta^{2}}{\sqrt{(x-\xi)^{2}+(y-\eta)^{2}}} \mathrm{~d} \xi \mathrm{d} \eta$ so that $u(x)=x^{2}+y^{2}$, where $(x, y) \in E$, is the exact solution to (5.44). For the uncompressed solution, the linear system is solved by stand Gaussian elimination method, and the number of subdivision $m_{n}$ used in the quadrature is taken as $m_{n}=40$. While, for the compressed solution, the linear system is solved by Gauss-Seidel type iterative method presented in Section 4.8 (number of iteration=20), and the number of subdivision used in quadrature is $m_{n}=80$. In Example 6.5 and Example 6.6, we consider the quadrangle $E$ with four vertices $(0,0),(1,0),(2,1.2),(0.5,1)$ (see Fig. 6.1).

Example 6.5 In this example, for the compressed solution, the truncation strategy 2 with $\nu=1$ is applied. The computational results are listed in Table 6.5.

Example 6.6 In this example, for the compressed solution, the block truncation strategy with parameters $p_{i^{\prime} i}=\sqrt{3}$ is applied. The computational results are listed in Table 6.6. 

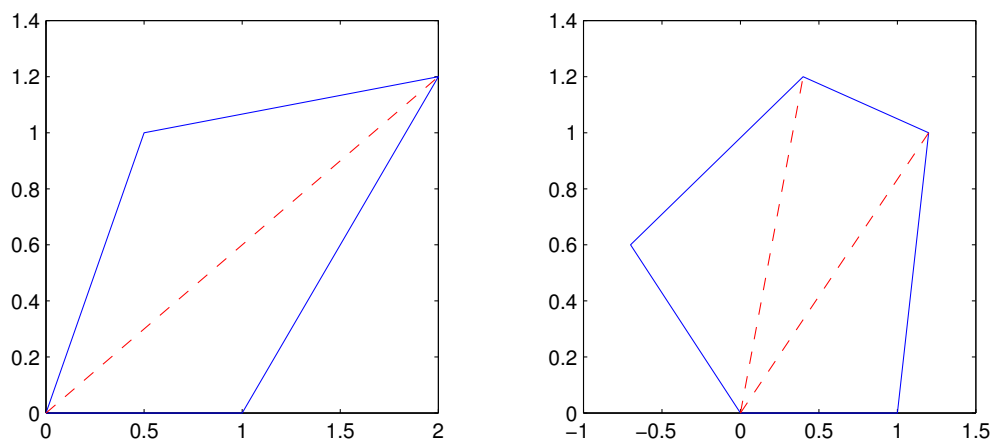

Figure 6.1: Computational domains of Example 6.5 and Example 6.7

Results for the integral equation over a quadrangle: strategy 2 with $\nu=1$

\begin{tabular}{|c|c|c|c|c|c|c|c|}
\hline & \multicolumn{3}{|c|}{ full collocation solution $u_{n}$} & \multicolumn{3}{|c|}{ compressed solution $\tilde{u}_{n}$} & \\
\hline$n$ & $m_{n}$ & $L^{\infty}$-error & order & $m_{n}$ & $L^{\infty}$-error & order & compression rate \\
\hline 1 & 40 & $3.607234 \mathrm{e}-1$ & & 40 & $3.607234 \mathrm{e}-1$ & & 1.000000 \\
$(2,0)$ & 40 & $9.443242 \mathrm{e}-2$ & 1.9335 & 80 & $9.443194 \mathrm{e}-2$ & 1.9335 & 1.000000 \\
$(3,0)$ & 40 & $2.282575 \mathrm{e}-2$ & 2.0486 & 80 & $2.282116 \mathrm{e}-2$ & 2.0489 & 0.894531 \\
$(4,0)$ & 40 & $5.735749 \mathrm{e}-3$ & 1.9926 & 80 & $5.753102 \mathrm{e}-3$ & 1.9880 & 0.520172 \\
$(5,0)$ & 40 & $1.456519 \mathrm{e}-3$ & 1.9775 & 80 & $1.449515 \mathrm{e}-3$ & 1.9888 & 0.227601 \\
$(6,0)$ & & & & 80 & $4.190603 \mathrm{e}-4$ & 1.7903 & 0.085734 \\
\hline
\end{tabular}

Table 6.5: Comparison of solutions obtained by the full collocation method and by our compressed collocation method (strategy 2, on a quadrangle). 
Results for the integral equation over a quadrangle: block truncation with $p_{i^{\prime} i}=\sqrt{3}$

\begin{tabular}{|c|c|c|c|c|c|c|c|}
\hline & \multicolumn{3}{|c|}{ full collocation solution $u_{n}$} & \multicolumn{3}{|c|}{ compressed solution $\tilde{u}_{n}$} & \\
\hline & $m_{n}$ & $L^{\infty}$-error & order & $m_{n}$ & $L^{\infty}$-error & order & compression rate \\
\hline 1 & 40 & $3.607234 \mathrm{e}-1$ & & 40 & $3.607234 \mathrm{e}-1$ & & 1.000000 \\
$(2,0)$ & 40 & $9.443242 \mathrm{e}-2$ & 1.9335 & 80 & $9.443194 \mathrm{e}-2$ & 1.9335 & 1.000000 \\
$(3,0)$ & 40 & $2.282575 \mathrm{e}-2$ & 2.0486 & 80 & $2.272426 \mathrm{e}-2$ & 2.0550 & 0.758301 \\
$(4,0)$ & 40 & $5.735749 \mathrm{e}-3$ & 1.9926 & 80 & $5.891051 \mathrm{e}-3$ & 1.9476 & 0.416626 \\
$(5,0)$ & 40 & $1.456519 \mathrm{e}-3$ & 1.9775 & 80 & $1.492354 \mathrm{e}-3$ & 1.9809 & 0.181021 \\
$(6,0)$ & & & & 80 & $9.469365 \mathrm{e}-4$ & 0.6563 & 0.067701 \\
\hline
\end{tabular}

Table 6.6: Comparison of solutions obtained by the full collocation method and by our compressed collocation method (block truncation, on a quadrangle).

In Example 6.7 and Example 6.8, we consider the pentagon $E$ with five vertices $(0,0),(1,0)$, $(1.2,1),(0.4,1.2),(-0.7,0.6)$ (see Fig. 6.1).

Example 6.7 In this example, for the compressed solution, the truncation strategy 2 with $\nu=1$ is applied. The computational results are listed in Table 6.7.

Example 6.8 In this example, for the compressed solution, the block truncation strategy with parameters $p_{i^{\prime} i}=\sqrt{3}$ is applied. The computational results are listed in Table 6.8.

\subsection{Parallel Computing}

As we see, when the level of the resolution is higher (for our case, when $n>7$ ), even a sparse storage scheme is adopted, due to the fast increasing of nonzero elements, the computation can not be fulfilled on one stand alone computer. The problem encountered is the limit of the physical memory of the available machine. The other problem is the painful lengthy computing time when the level is getting higher. Therefore if we want to compute higher level's solution, one definitely good answer is to appeal to parallel computing.

To achieve parallel computing using methods proposed in previous chapters, one must con- 
Results for the integral equation over a pentagon: strategy 2 with $\nu=1$

\begin{tabular}{|c|c|c|c|c|c|c|c|}
\hline & \multicolumn{3}{|c|}{ full collocation solution $u_{n}$} & \multicolumn{3}{|c|}{ compressed solution $\tilde{u}_{n}$} \\
$n$ & $m_{n}$ & $L^{\infty}$-error & order & $m_{n}$ & $L^{\infty}$-error & order & compression rate \\
\hline 1 & 40 & $2.015684 \mathrm{e}-1$ & & & $2.0157 \mathrm{e}-1$ & & 1.000000 \\
$(2,0)$ & 40 & $4.076680 \mathrm{e}-2$ & 2.3058 & 80 & $4.076633 \mathrm{e}-2$ & 2.3058 & 1.000000 \\
$(2,1)$ & 40 & $1.017880 \mathrm{e}-2$ & 2.0018 & 80 & $1.017623 \mathrm{e}-2$ & 2.0022 & 0.894531 \\
$(2,2)$ & 40 & $2.593854 \mathrm{e}-3$ & 1.9724 & 80 & $2.596571 \mathrm{e}-3$ & 1.9705 & 0.520172 \\
$(2,3)$ & 40 & $6.440248 \mathrm{e}-4$ & 2.0099 & 80 & $6.434862 \mathrm{e}-4$ & 2.0126 & 0.227601 \\
$(2,4)$ & & & & 80 & $1.587823 \mathrm{e}-4$ & 2.0189 & 0.085734 \\
$(2,5)$ & & & & 80 & $5.511457 \mathrm{e}-5$ & 1.5265 & 0.030162 \\
\hline
\end{tabular}

Table 6.7: Comparison of solutions obtained by the full collocation method and by our compressed collocation method (strategy 2, on a pentagon).

Results for the integral equation over a pentagon: block truncation with $p_{i^{\prime} i}=\sqrt{3}$

\begin{tabular}{|c|c|c|c|c|c|c|c|}
\hline & \multicolumn{3}{|c|}{ full collocation solution $u_{n}$} & \multicolumn{4}{|c|}{ compressed solution $\tilde{u}_{n}$} \\
$n$ & $m_{n}$ & $L^{\infty}$-error & order & $m_{n}$ & $L^{\infty}$-error & order & compression rate \\
\hline 1 & 40 & $2.015684 \mathrm{e}-1$ & & & $2.0157 \mathrm{e}-1$ & 40 & 1.000000 \\
$(2,0)$ & 40 & $4.076680 \mathrm{e}-2$ & 2.3058 & 80 & $4.076633 \mathrm{e}-2$ & 2.3058 & 1.000000 \\
$(2,1)$ & 40 & $1.017880 \mathrm{e}-2$ & 2.0018 & 80 & $1.019395 \mathrm{e}-2$ & 1.9997 & 0.758301 \\
$(2,2)$ & 40 & $2.593854 \mathrm{e}-3$ & 1.9724 & 80 & $2.624460 \mathrm{e}-3$ & 1.9576 & 0.416626 \\
$(2,3)$ & 40 & $6.440248 \mathrm{e}-4$ & 2.0099 & 80 & $6.675855 \mathrm{e}-4$ & 1.9750 & 0.181021 \\
$(2,4)$ & & & & 80 & $1.939918 \mathrm{e}-4$ & 1.7830 & 0.067701 \\
$(2,5)$ & & & & 80 & $9.054316 \mathrm{e}-5$ & 1.0993 & 0.023000 \\
\hline
\end{tabular}

Table 6.8: Comparison of solutions obtained by the full collocation method and by our compressed collocation method (block truncation, on a pentagon). 
sider two factors:

- to be able to simultaneously generate the coefficient matrix of the discrete linear system, henceforth the coefficient matrix needs to be partitioned into pieces distributed on different machines

- to be able to simultaneously solve the resulting linear system using the distributed matrix on different machines

It turns out that we can easily design our algorithm, taking advantage of both sparse storage and parallel computing. The main idea is that we will partition the coefficient matrix along row direction. Thus on different machines, disjoint partitions of the matrix are simultaneously computed. To solve the linear system, we adopt the Gauss-Seidel type multilevel iteration methods proposed in Section 4.8. The algorithm is as follows:

\section{A Parallel computing algorithm:}

1. Allocate the computing loads roughly evenly to different computers. Let $n p \geq 2$ be the number of processes. Compute the quota of rows

$$
Q=\left\lfloor\frac{f(n)}{n p}\right\rfloor
$$

where, as before $f(n)$ is the dimension of the approximation space $\mathbb{F}_{n}$, i.e., the order of the matrix $\mathbf{A}_{n}=\mathbf{K}_{n}-\mathbf{E}_{n}$. Remember we are trying to generate and solve the linear system (2.18) or (4.102). Recall we denote elements of $\mathbf{K}_{n}$ by $K_{i^{\prime} j^{\prime}, i j}$, elements of $\mathbf{E}_{n}$ by $E_{i^{\prime} j^{\prime}, i j}$, and elements of $\mathbf{f}_{n}$ by $f_{i^{\prime} j^{\prime}}$, where $\left(i^{\prime}, j^{\prime}\right),(i, j) \in \mathbb{U}_{n}$. Let $r_{i}$ be the number of rows to be allocated for process $i=0,1, \cdots, n p-1$, then

$$
r_{i}=Q, \text { for } \quad i \in \mathbb{Z}_{n p-1} \quad \text { and } \quad r_{n p-1}=f(n)-\sum_{i=0}^{n p-2} r_{i}
$$

2. Simultaneously compute nonzero coefficient elements $K_{i^{\prime} j^{\prime}, i j}$ and $E_{i^{\prime} j^{\prime}, i j}$ (by applying matrix compression) and the right handed vector $\mathbf{f}_{n}$ on each distributed machines. No communication between the computing nodes is needed at this step.

As we know, any element $K_{i^{\prime} j^{\prime}, i j}$ and $E_{i^{\prime} j^{\prime}, i j}$ are completely determined by its index 
$\left(i^{\prime} j^{\prime}, i j\right)$. Denote the algorithm to compute $K_{i^{\prime} j^{\prime}, i j}$ by $\operatorname{Kelt}\left(i^{\prime}, j^{\prime}, i, j\right)$, the algorithm to compute $E_{i^{\prime} j^{\prime}, i j}$ by $\operatorname{Eelt}\left(i^{\prime}, j^{\prime}, i, j\right)$, and the algorithm to compute $f_{i^{\prime} j^{\prime}}$ by $\operatorname{felt}\left(i^{\prime}, j^{\prime}\right)$. Note differences between local indices of matrix elements on each machine and the global indices of the elements in the original matrix. All algorithms $\operatorname{Kelt}\left(i^{\prime}, j^{\prime}, i, j\right)$, $\operatorname{Eelt}\left(i^{\prime}, j^{\prime}, i, j\right)$ and $\operatorname{felt}\left(i^{\prime}, j^{\prime}\right)$ assumes the global indices.

3. Employ the Gauss-Seidel multilevel iteration method (see Section 4.8) to solve the linear system simultaneously on different machines. Communications between nodes are assumed.

(a) Obtain the initial solution $\mathbf{x}_{0}$ on process 0 . Then Broadcast $\mathbf{x}_{0}$ to all other processes $1,2, \cdots, n p-1$.

(b) On each nodes, simultaneously do matrix-vector multiplication to obtain a locally updated partial solution $\mathbf{x}_{\nu}^{(i)}$, where, $i$ represents the index of process, $i \in \mathbb{Z}_{n p}$, and $\nu$ stands for the number of iteration, and $\nu>1$. Let $\mathbf{A}^{(i)}=\left[A_{j k}^{(i)}: j \in \mathbb{Z}_{r_{i}}, k \in\right.$ $\left.\mathbb{Z}_{f(n)}, i \in \mathbb{Z}_{n p}\right]$ be the partial matrix computed on process $i$, and $\mathbf{x}_{\nu}^{(i)}=\left[x_{\nu}^{(i)}(j)\right.$ : $\left.j \in \mathbb{Z}_{f(n)}, i \in \mathbb{Z}_{n p}\right]$ be the local solutions on process $i$, and $\mathbf{f}^{(i)}=\left[f_{j}^{(i)}: j \in \mathbb{Z}_{r_{i}}\right]$. Then for each $\nu>1$,

$$
x_{\nu}^{(i)}(j)=f_{j}^{(i)}+\sum_{k=0}^{f(n)-1} A_{j k}^{(i)} x_{\nu-1}(k), \quad j \in \mathbb{Z}_{r_{i}},
$$

where $i \in \mathbb{Z}_{n p}$, and $\mathbf{x}_{\nu-1}=\left[x_{\nu-1}(k): k \in \mathbb{Z}_{f(n)}\right]$ is the previous computed global solution.

(c) Assemble the updated solution $\mathbf{x}_{\nu}$ on node 0 from the local partial segments $\mathbf{x}_{\nu}^{(i)}, i \in \mathbb{Z}_{n p}$ and compute the current approximation error.

(d) Broadcast the newest solution $\mathbf{x}_{\nu}$ to all other nodes in the current communicator.

(e) Do step 3b-step 3d again until satisfied solution is achieved.

All the examples using above parallel algorithm are computed on energy.cluster.wvu.edu. The cluster has one frontend node and 27 computing nodes. Each node has 1.2 Ghz Pentium, Dual processors, 1 Gbyte of memory shared by the two processors and 18 Gbyte of disk and has a $100 \mathrm{Mb}$ ethernet interconnect. 
Example 6.9 To demonstrate the ideas proposed in the above algorithm, we solve the integral equation (6.1) again using parallel algorithm. For the example of $n=7$, compression is assumed. While for the example of $n=5$, no compression is assumed. The linear system is solved by Gauss-Seidel type multilevel iteration method. All the entries are computed by analytical formula. We tabulate in Table 6.9 the computing time for different number of processes np. $t_{m}$ is the average time consumed by each process to generate the sparse coefficient matrix. $t_{s}$ is the average time spent by each process to solving the linear system by Gauss-Seidel type multilevel iteration method. When $n=7$, the computing requires the same number of computing nodes as number of processes $n p$. While when $n=5$, we require all the two processors on each node.

The results are also plotted in Figure 6.2 and Figure 6.3. From both figures, we clearly see, as the number of processes increase, the average time $t_{m}$ needed for each process to generate the partial matrix is decreasing. As described in the parallel computing algorithm, no communication between nodes is assumed for this purpose. While, the average time needed for each process to solve the linear system by iteration method (matrix-vector multiplication) is more or less the same as when there is only one process (the serial computing). This is as expected. The reason is as follows:

The matrix-vector multiplication time needed in each process only differs by a very insignificant factor when np increases. While more and more communications among processes are caused due to more processes are introduced into the whole communicator. The increased communication time more or less offset the reduced time for matrix-vector multiplication. Therefore, almost equal time for solving the system observed when different number of processes are required. The fluctuation of each curve is apparently due to the dynamical load of the cluster. Since a number of other jobs are also simultaneously running in the cluster. I imagine that if only one job is running in the cluster before it's done, then the fluctuation won't be observed. 


\begin{tabular}{|c|c|c|c|c|}
\hline \multicolumn{3}{|c|}{ level $n=7$} & \multicolumn{2}{|c|}{ level $n=5$} \\
\hline $\mathrm{np}$ & $t_{m}(\mathrm{sec})$ & $t_{s}(\mathrm{sec})$ & $t_{m}(\mathrm{sec})$ & $t_{s}(\mathrm{sec})$ \\
\hline 1 & 14023 & 1349 & 917 & 103 \\
\hline 2 & 5101 & 1345 & 687 & 140 \\
\hline 3 & 2934 & 1317 & 406 & 134 \\
\hline 4 & 2258 & 2611 & 229 & 89 \\
\hline 5 & 2335 & 2122 & 256 & 131 \\
\hline 6 & 1282 & 1289 & 204 & 136 \\
\hline 7 & 1069 & 1283 & 205 & 172 \\
\hline 8 & 891 & 1281 & 143 & 85 \\
\hline 9 & 793 & 1352 & 148 & 85 \\
\hline 10 & 713 & 1275 & 120 & 127 \\
\hline 11 & 648 & 1274 & 121 & 127 \\
\hline 12 & 594 & 1272 & 102 & 127 \\
\hline 13 & 548 & 1271 & 92 & 84 \\
\hline 14 & 509 & 1270 & 84 & 84 \\
\hline 15 & 539 & 1902 & 85 & 84 \\
\hline 16 & 471 & 1268 & 71 & 84 \\
\hline 17 & 443 & 1266 & 73 & 87 \\
\hline 18 & 418 & 1267 & 59 & 167 \\
\hline 19 & 440 & 1894 & 62 & 84 \\
\hline 20 & 415 & 1892 & 50 & 84 \\
\hline 21 & 367 & 1264 & & \\
\hline 22 & 375 & 1890 & & \\
\hline 23 & 361 & 1891 & & \\
\hline 24 & 321 & 1263 & & \\
\hline
\end{tabular}

Table 6.9: Comparison of times consumed when number of processes varies. 


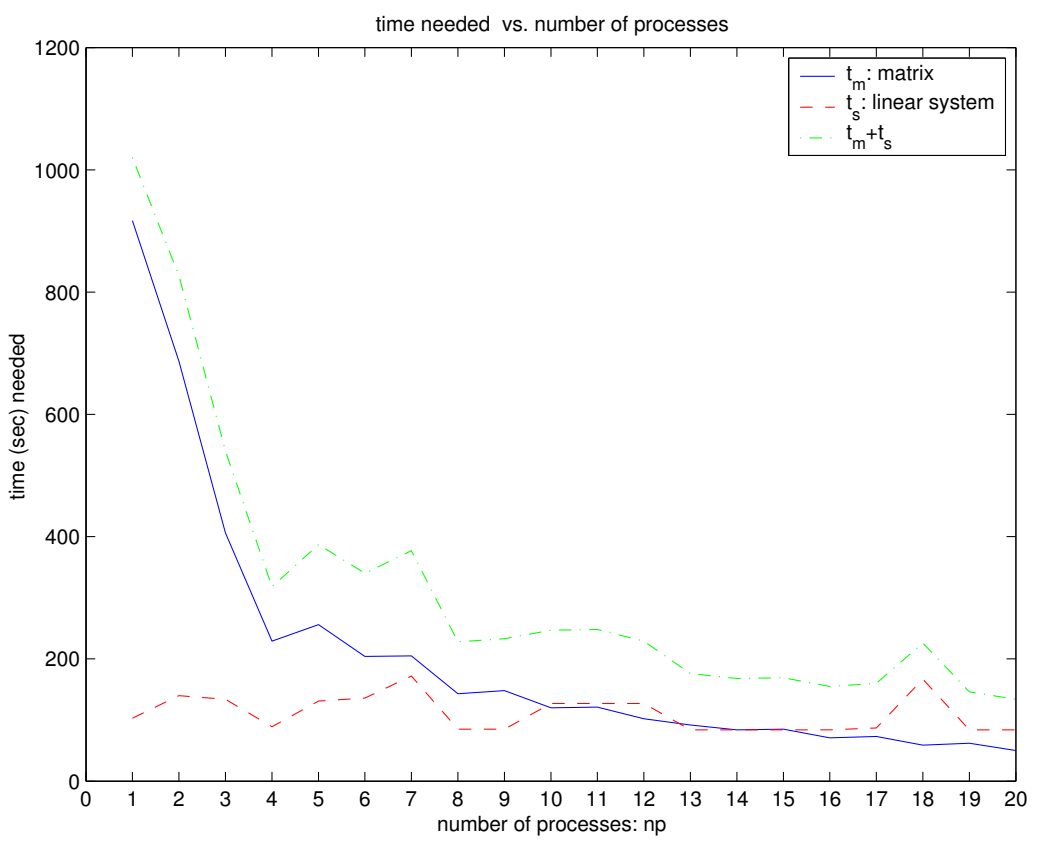

Figure 6.2: Time needed vs. number of processes: level $n=5$

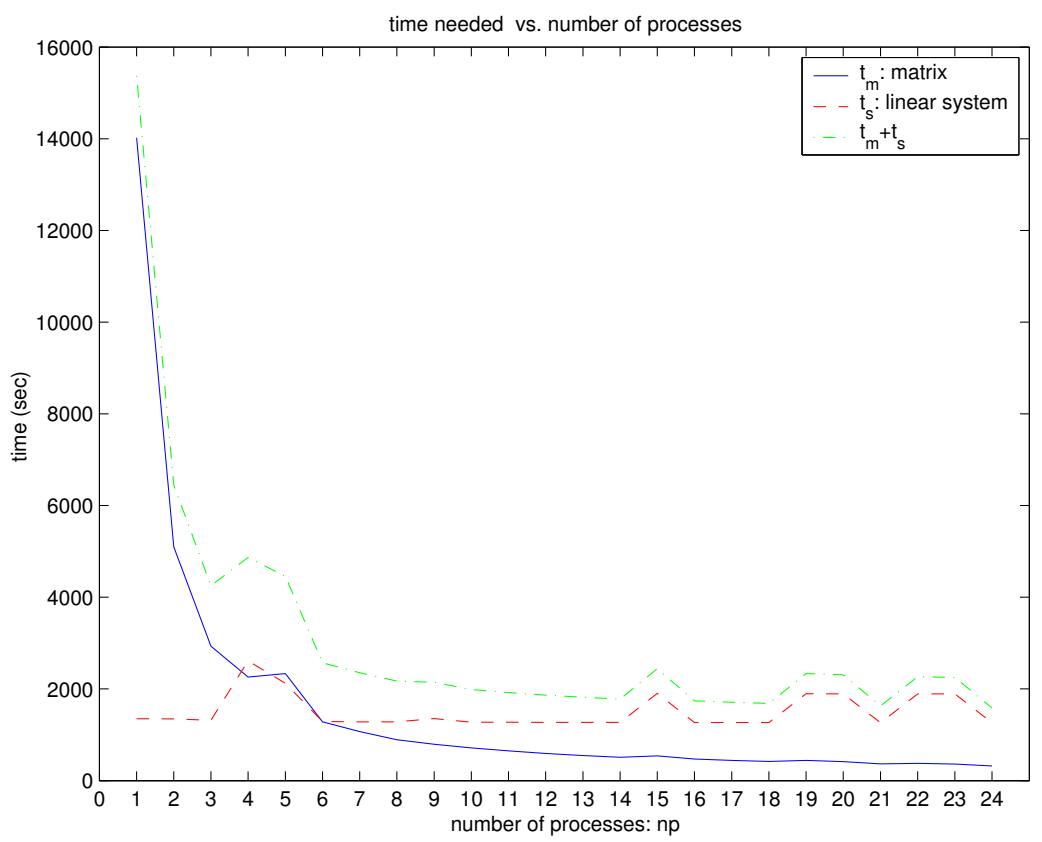

Figure 6.3: Time needed vs. number of processes: level $n=7$ 


\section{Bibliography}

[Al] B.K.AlPERT, A class of bases in $L^{2}$ for the sparse representation of integral operators, SIAM J. Math. Anal., 24 (1993), pp. 246-262

[An] P.M. Anselone, Collectively Compact Operator Approximation Theorey and Applications to Integral Equations, Prentice-Hall, Englewood Cliffs, NJ, 1971

[At1] K. E. Atkinson, A survey of boundary integral equation methods for the numerical solution of Laplace's equation in three dimensions, in Numerical Solution of Integral Equations, M. Golberg, ed., Plenum Press, NY 1990, pp. 1-34

[At2] K. E. Atkinson, The Numerical Solution of Integral Equations of Second Kind, Cambridge University Press, Cambridge, 1997

[AC1] K.E. Atkinson And D. Chien, Piecewise polynomial collocation for boundary integral equations, SIAM J. Sci. Comput., 16 (1994), pp. 651-681

[AC2] K.E. Atkinson And D. Chien, A fast matrix-vector multiplication method for solving the radiosity equation, Adv. Comput. Math., 12 (2000), pp. 151-174

[ACr] K.E. Atkinson And G. Chandler, The collocation method for solving the radiosity equation for unoccluded surfaces, J. Integral Equations Appl., 10 (1998), pp. 253-290

[AGS] K. E. Atkinson, I. Graham and I. Sloan, Piecewise continuous collocation for integral equations, SIAM J. Numer. Anal., 20 (1983), 172-186 
[BCR] G. Beylkin, R. Coifman And V. Rokhlin, Fast wavelet transforms and numerical algorithms I, Comm. Pure Appl. Math., 44 (1991), pp. 141-183

[CMX1] Z. Chen, C. A. Micchelli And Y. Xu, The Petrov-Galerkin methods for second kind integral equations II: Multiwavelet scheme, Adv. Comput. Math., 7 (1997), pp. 199-233

[CMX2] Z. Chen, C. A. Micchelli And Y. XU, A construction of interpolating wavelets on invariant sets, Math. Comp., 68 (1999), pp. 1569-1587

[CMX3] Z. Chen, C. A. Micchelli And Y. Xu, Fast Collocation Methods for Second Kind Integral Equations, SIAM J. NUMER. ANAL., Vol. 40, No. 1, pp. 344-375

[CMX4] Z. Chen, C. A. Micchelli And Y. Xu, A multilevel Method for Solving Operator Equations, J. Math. Anal. and Appl. 262, 688-699 (2001)

[CMX5] Z. Chen, C. A. Micchelli And Y. Xu, Discrete wavelet Petrov-Galerkin methods, Adv. Comput. Math., 16 (2002), pp. 1-28

[CMX6] Z. Chen, C. A. Micchelli And Y. Xu, A multilevel Method for Solving Operator Equations, J. Math. Anal. and Appl. 262, 688-699 (2001)

[CWX1] Z. Chen, B. WU, AND Y. XU, Multilevel augmentation methods for solving operator equations, submitted

[CWX2] Z. Chen, B. Wu And Y. Xu, An Implementation of Fast Collocation Methods for Integral Equations, preprint

[CX] Z. Chen And Y. Xu, The Petrov-Galerkin and iterated Petrov-Galerkin methods, Adv. Comput. Math., 16 (2002), pp. 1-28

[DKPS] W. Dahmen, B. Kleemann, S. Prössdorf and R. Schneider, A multiscale method for the double layer potential equation on a polyhedron, in Advances in Computational Mathematics: New Delhi, India, eds. H. P. Dikshit and C. A. Micchelli, World Scientific, Singapore, 1994. 
[DPS1] W. Dahmem, S. Proessdorf, And R. Schineider, Wavelet approximation methods for pseudodifferential equations II: matrix compression and fast solutions, Adv. Comput. Math., 1 (1993), pp. 259-335

[DPS2] W. Dahmen, S. Prössdorf And R. Schneider, Wavelet approximation methods for psuedodifferential equations II: Matrix compression and fast solutions, Adv. Comput. Math., 1 (1993), 259-335.

[FMX] W. FAng, F. MA AND Y. XU, Multilevel Iteration Methods for solving Integral Equations of the Second Kind, J. Integral Equations Appl., (to appear)

[FWX] W. FAng, Y. WANG AND Y. XU, An Implementation of Fast Wavelet Galerkin Methods for Integral Equations of the Second Kind, J. Scientific Computing (to appear)

[H] J.E. Hutchinson, Fractals and self similarity, India Univ. Math. J. 30 (1981), 713-747.

[K] R. KREss, Linear Integral Equations, 2nd ed., Springer-Verlag, New York, 1999

[KX] H. Kaneko AND Y. XU, Gauss-Type Quadratures for Weakly Singular Integrals and Their Application to Fredholm Integral Equations of the Second Kind, Math. Comp. 62 (1994) 739-753

[MSX1] C. A. Micchelli, T. Sauer And Y. Xu , A construction of refinable sets for interpolating wavelets, Result. Math. 34 (1998) 359-372

[MSX2] C. A. Micchelli, T. Sauer AND Y. XU, Subdivision schemes for iterated funcion systems,, Proc. Amer. Math. Socl, 129 (2001), pp. 1861-1872

[MX1] C. A. Micchelli, AND Y. XU , Using the matrix refinement equation for the construciton of wavelets on invariant sets, Appl, Comput. Harmon. Anal., 1 (1994), pp. 391-401 
[MX2] C. A. Micchelli, AND Y. XU, Reconstruction and decomposition algorithms for biorthogonal multiwavelets, Multidimen. Systems Signal Process., 8 (1997), pp. 31-69

[MXZ] C. A. Micchelli, Y. Xu AND Y. ZhaO, Wavelet Garlerkin Methods for SecondKind Integral Equations, J. Comp. and Appl. Math. 86 (1997) 251-270

[PS] T. Von PetersdorfF And C. Schwab, Wavelet approximation of first kind integral equations in a polygon, Numer. Math., 74 (1996), pp. 479-516

[PSS] T. Von Petersdorff, C. Schwab, And R. Schneider, Multiwavelets for second-kind integral equations, SIAM J. Numer. Anal., 34 (1997), pp. 2212-2227

[R] A. RATHSFELD, A wavelet algorithm for the solution of a singular integral equaiton over a smooth two-dimensional manifold, J. Integral Equaitons Appl., 10 (1998), pp. $445-501$

[Ri] J. R. RICE, On the degree of convergence of nonlinear spline approximaton, Approx. with Emphasis on Spline Functions (I.J. Schoenberg, ed.), Academic Press, New York, 1969, pp. 349-365

[XZ] Y. XU AND Y. ZhaO, An Extrapolation Method for a Class of Boundary Integral Equations, Math. Compu. 65 (1996), 587-610 